\title{
The ideal of relations for the ring of invariants of $n$ points on the line
}

Received November 11, 2009

\begin{abstract}
The ring of projective invariants of $n$ ordered points on the projective line is one of the most basic and earliest studied examples in Geometric Invariant Theory. It is a remarkable fact and the point of this paper that, unlike its close relative the ring of invariants of $n$ unordered points, this ring can be completely and simply described. In 1894 Kempe found generators for this ring, thereby proving the First Main Theorem for it (in the terminology introduced by Weyl). In this paper we compute the relations among Kempe's invariants, thereby proving the Second Main Theorem (again in the terminology of Weyl), and completing the description of the ring 115 years later.

This paper introduces a number of new tools to the problem, and uses the graphical algebra formalism to intermediate between representation-theoretic arguments (for symmetric and Lie groups), and the symmetry-breaking of the Speyer-Sturmfels degeneration.
\end{abstract}

\section{Contents}

1. Introduction . . . . . . . . . . . . . . . . . . . . . 1

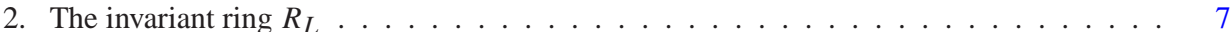

3. The toric degenerations $\operatorname{gr}_{\Xi} S_{L}$ and $\operatorname{gr}_{\Xi} R_{L} \ldots \ldots \ldots \ldots \ldots$

4. The toric ideal is generated by quadratics and toric generalized Segre cubics . . . . . . . 16

5. The ideal is generated by quadratics and generalized Segre cubics . . . . . . . . . . . . . 24

6. The structure of $V_{L}$ and its tensor powers . . . . . . . . . . . . . . . . . . . . . . . . 29

7. Retrogeneration of the ideal . . . . . . . . . . . . . . . . . . . . 38

8. The ideal is generated by quadratics . . . . . . . . . . . . . . . . . . . . . . . . . . . . . 48

9. The quadratics are generated by the simplest binomials . . . . . . . . . . . . . . 57

\section{Introduction}

We consider the ring of invariants of $n$ points on the projective line, defined as the projective coordinate ring of the GIT quotient of $\left(\mathbb{P}^{1}\right)^{n}$ by the group SL(2). This is a classical

B. Howard: Department of Mathematics, University of Michigan, 2074 East Hall, 530 Church Street, Ann Arbor, MI 48109-1043, USA; e-mail: howardbj@umich.edu

J. Millson: Department of Mathematics, University of Maryland, Mathematics Building, College Park, MD 20742-4015, USA; e-mail: jjmillson@gmail.com

A. Snowden: Department of Mathematics, Massachusetts Institute of Technology, Headquarters Office, Building 2, Room 236, 77 Massachusetts Avenue,

Cambridge, MA 02139-4307, USA; e-mail: awsnowden@gmail.com

R. Vakil: Department of Mathematics, Stanford University, Stanford, CA 94305, USA; e-mail: vakil@math.stanford.edu 
archetype of GIT and a common first example in the theory. To form the quotient, one must choose weights $w=\left(w_{1}, \ldots, w_{n}\right)$; it is then given by

$$
\left(\mathbb{P}^{1}\right)^{n} \rightarrow\left(\mathbb{P}^{1}\right)^{n} / / w \operatorname{SL}(2)=\operatorname{Proj} R_{w}
$$

where $R_{w}=\bigoplus_{k=0}^{\infty} R_{w}^{(k)}$, with $R_{w}^{(k)}=\Gamma\left(\left(\mathbb{P}^{1}\right)^{n}, \mathcal{O}\left(k w_{1}, \ldots, k w_{n}\right)\right)^{\mathrm{SL}(2)}$. (Note that we use the notation $R^{(k)}$ for the $k$ piece of a graded ring $R$.) The ring of invariants $R_{w}$ turns out to be generated in its lowest nonzero degree, so the GIT quotient has a natural projective embedding. We denote the weight $(1, \ldots, 1)$ by $1^{n}$.

Theorem 1.1 (Main Theorem, informal version). If $w \neq 1^{6}$, the ideal of relations between lowest degree invariants is generated by quadratics.

Detailed motivation and background for this problem are given in the announcement [HMSV2]. We describe there how small cases have long been known to yield beautiful classical geometry. In this paper, we show that this rich structure extends to any number of points with any weighting: the relations for the moduli space are generated by a particularly simple type of quadratic, with the single exception of the Segre cubic for six points. In a precise sense, the ideal is cut out by essentially one equation, inherited from the $n=8$ case. If Kempe's theorem is the analogue of Weyl's "First Main Theorem" for $\mathrm{SL}(2)$ (see $[\mathrm{W}]$ ), then this is the analogue of his "Second Main Theorem."

This is the culmination of a number of papers on this topic, so we wish to be clear about its relationship to the others. [HMSV1] (subsuming two earlier arXiv preprints) opened up this question: through blunt toric degeneration and explicit local calculation, hints that the relations were simple quadrics were observed. It was shown there that certain quadrics (not as simple as those in this paper) cut out the space. The other important paper [HMSV6] is on the interplay of the geometry and representation theory in the modular fivefold parametrizing eight points on the line.

The current paper is the main one of the series. It introduces fundamentally new techniques to the problem, and is not simply a refinement of our preliminary ideas of [HMSV1]. The quadric here is simpler than those speculated about in [HMSV1]. The argument uses the formalism of graphical algebras to balance on one hand representation theory arguments (of both symmetric and Lie groups), with on the other hand information from symmetry-breaking (from a better toric degeneration, discovered by Speyer and Sturmfels from tropical motivation).

\subsection{Integrality issues}

For simplicity, we prove our results over $\mathbb{Q}$, but our results apply more generally, as we show in [HMSV4]. In preparation for this, we prove intermediate results over more general base rings, which we hope will not distract readers interested in characteristic 0 . In particular, this paper proves Theorem 1.1 over $\mathbb{Z}[1 / 12$ !], but the precise version (Theorem 1.2) only over $\mathbb{Q}$. 


\subsection{The graphical formalism}

We use a graphical interpretation of the ring of invariants, which allows us to deal effectively with both the $\mathfrak{S}_{n}$-symmetries and the broken symmetries of toric degenerations. To a directed graph $\Gamma$ on vertices labeled 1 through $n$ (in bijection with the points), with valence vector $k w=\left(k w_{1}, \ldots, k w_{n}\right)$, we associate an invariant in $R_{w}^{(k)}$ :

$$
X_{\Gamma}=\prod_{\overrightarrow{i j}}\left(x_{i} y_{j}-x_{j} y_{i}\right) \text {. }
$$

Here the product is taken over the edges $\overrightarrow{i j}$ of $\Gamma$ and $x_{i}$ and $y_{i}$ are projective coordinates for $\mathbb{P}^{1}$. Note that if $\Gamma$ has a loop-that is, an edge with the same source and targetthen $X_{\Gamma}=0$. (Throughout this paper, graphs are allowed to have loops and multiple edges between the same vertices.) A fundamental result from classical invariant theory states that the $X_{\Gamma}$ span $R_{w}^{(k)}$. Kempe showed that for $w=1^{n}$ and $n$ even the ring $R_{w}$ is generated in degree 1 (Theorem 2.1). Thus, in this situation, $R_{w}$ is generated by those $X_{\Gamma}$ where $\Gamma$ is a matching, that is, a graph in which each vertex belongs to precisely one edge. A similar result holds for any $n$ and $w$.

\subsection{Relations}

The theorem of Kempe mentioned above provides generators for the ring $R_{w}$. The purpose of this paper is to determine the relations between these generators. A number of obvious relations exist; we catalog some of them below. An important phenomenon shows itself already in these simple examples: relations on $n$ points can be extended to give relations on more than $n$ points. This is a key theme in our treatment and is discussed in detail in $\$ 7.1$.

The most obvious relation is the sign relation: we have $X_{\overrightarrow{a b}}=-X_{\overrightarrow{b a}}$. This can be regarded as a relation between two invariants with $n=2$ and $w=1^{2}$. It extends to invariants with arbitrary $n$ and $w$ as follows: if $\Gamma$ is any graph and $\Gamma^{\prime}$ is obtained from $\Gamma$ by switching the direction of a single edge then $X_{\Gamma}=-X_{\Gamma^{\prime}}$.

The next most simple relation appears when $n=4$ and $w=1^{4}$ :

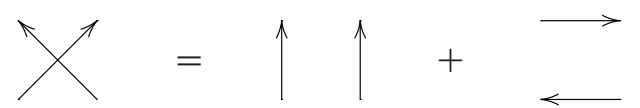

This is the classical Plücker relation. It extends to each $R_{w}^{(k)}$ as well: given a graph $\Gamma$ with valence vector $k w$ and two edges $\overrightarrow{a b}$ and $\overrightarrow{c d}$ of $\Gamma$ we have the identity

$$
X_{\Gamma}=X_{\Gamma_{1}}+X_{\Gamma_{2}}
$$

where $\Gamma_{1}$ and $\Gamma_{2}$ are the graphs obtained from $\Gamma$ by replacing $\{\overrightarrow{a b}, \overrightarrow{c d}\}$ with $\{\overrightarrow{a d}, \overrightarrow{c b}\}$ and $\{\overrightarrow{a c}, \overrightarrow{b d}\}$ respectively. We will use the phrase "to Plücker two edges $\overrightarrow{a b}$ and $\overrightarrow{b c}$ of a graph $\Gamma$ " to mean "to replace $X_{\Gamma}$ by $X_{\Gamma_{1}}+X_{\Gamma_{2}}$." The sign and Plücker relations are both linear relations; in fact, they span all linear relations. 
Some higher degree relations are conveniently thought of in terms of colored graphs. By a $k$-colored graph we mean a graph in which each edge has been assigned one of $k$ colors. By a multi-matching of degree $k$ we mean a $k$-colored graph in which each vertex appears in precisely one edge of each color. Let $\Gamma$ be a multi-matching of degree $k$ on $n$ vertices. We define the element $X_{\Gamma}$ of $\left(R_{1^{n}}^{(1)}\right)^{\otimes k}$ to be the pure tensor $\otimes X_{\Gamma(i)}$ where the product is over the colors $i$ and $\Gamma(i)$ is the subgraph of $\Gamma$ on the edges of color $i$. In terms of colored graphs, the map $\left(R_{1^{n}}^{(1)}\right)^{\otimes k} \rightarrow R_{1^{n}}^{(k)}$ "forgets the color."

The Segre cubic relation is described with colored graphs as follows:

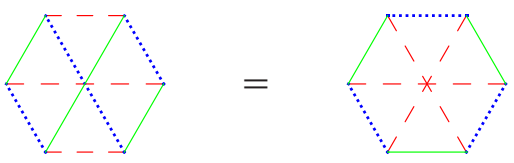

(Because the paper version of the article is black-and-white, while the online publication is in color, we will follow the convention that the solid lines are green, the dashed red and the dotted blue.) Each edge should be directed in the same way on both sides of the equation. This relation holds because the graphs on each side have the same set of edges - only the colors are different. The Segre cubic relation extends to cubic relations on $R_{1^{n}}^{(1)}$ for any even $n>6$. For example, we have the following relation on eight points starting from the Segre cubic on six points:

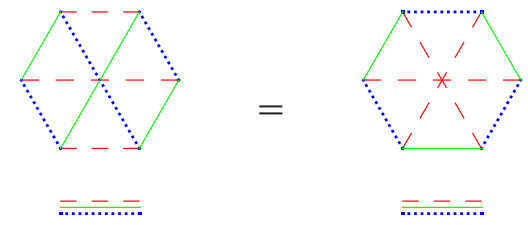

There is a "new" relation for $n=8$, binomial and quadratic:

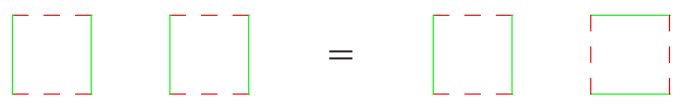

As with all previously discussed relations, the above relation extends to relations on more points. One way to extend the above relation is to add some number of doubled edges to each side; we call such relations the simplest binomial relations. Examples are given in [HMSV2, §4].

\subsection{The main theorem}

We now state Theorem 1.1 more precisely in the main case of $n$ even and unit weights.

Theorem 1.2 (Main theorem, main case). For even $n \neq 6$ the ideal $I_{1^{n}}$ of relations (the kernel of $\operatorname{Sym} R_{1^{n}}^{(1)} \rightarrow R_{1^{n}}$ ) is generated by the simplest binomial relations. The symmetric group $\mathfrak{S}_{n}$ acts transitively on these relations, so any one of them generates $I_{1^{n}}$ as an $\mathfrak{S}_{n}$-ideal. 
(The ideal $I_{1^{6}}$ is principal and generated by the Segre cubic relation (1.2) over $\mathbb{Z}$.)

Remark 1.3. The discussion of [HMSV1, §2.17] explains how to reduce the case of arbitrary weight to the "main case." Thus as a corollary we have Theorem 1.1, and more precisely, the quadratics are explicitly given by "clumping vertices" (loc. cit.). Thus for the remainder of the paper, we will deal only with this "main case" of $w=1^{n}$ and $n$ even.

We essentially conjectured Theorems 1.1 and 1.2 in [HMSV1, §1.5]. We saw the two main theorems of that paper as evidence: first, that a class of relations called the "simple binomial relations," containing the simplest binomial relations, cuts out the quotient scheme-theoretically, and second, that the ideal is generated by relations of degree at most four. These two results are subsumed by Theorem 1.2 in the main case, and Theorem 1.1 (in its more precise form, Remark 1.3) in general.

Remark 1.4. Given that Theorem 1.2 states that $I_{1^{n}}$, for even $n \neq 6$, is generated by a single quadratic up to $\mathfrak{S}_{n}$-symmetry, one may wonder whether the same holds when $n$ is odd. A short representation-theoretic argument shows that if $n$ is 5, 7 or 9 then this is indeed the case (see [HMSV2, Fig. 3(h)] for a simple generator with $n=5$ ), but if $n$ is odd and at least 11 then the space of quadratic relations is not a cyclic $\mathfrak{S}_{n}$-module, so $I_{1^{n}}$ is not principal as an $\mathfrak{S}_{n}$-ideal.

\subsection{Representation-theoretic description}

Here is a representation-theoretic description of the quadratics (in the main case) in characteristic 0 that is both striking and relevant. The $\mathfrak{S}_{n}$-representation on $R_{1^{n}}^{(1)}$ is irreducible and corresponds to the partition $n / 2+n / 2$. The representation $\operatorname{Sym}^{2} R_{1^{n}}^{(1)}$ is multiplicityfree and contains those irreducibles corresponding to partitions with at most four parts, all even (Proposition 6.5). The space of quadratic relations is the subspace of $\mathrm{Sym}^{2} R_{1^{n}}^{(1)}$ spanned by those irreducibles corresponding to partitions with precisely four parts. Being multiplicity-free, this is necessarily a cyclic $\mathfrak{S}_{n}$-module. The reader may wonder why we privilege a particular generator; the answer is that this relation is in some imprecise sense forced upon us by the graphical formalism.

\subsection{Outline of proof}

We now outline the proof, noting where the arguments are ad hoc or less satisfactory. The challenge is to relate three structures which often operate at cross purposes: the generation of new relations from relations on fewer points; the action of $\mathfrak{S}_{n}$ on everything; and the graphical description of the algebra, including the use of colored graphs to describe relations.

In $\S 2$, we set the stage by giving our preferred description of the invariant ring. We replace the integer $n$ by a finite set $L$ of cardinality $n$, as this makes many constructions more transparent. In \$3-4 we use a Speyer-Sturmfels toric degeneration to get some control on the degrees and types of generators, temporarily breaking the $\mathfrak{S}_{L}$-symmetry. We 
show that the degenerated ring is generated in degree one and that the relations between the degree one generators are generated by quadratic relations and certain explicit cubic relations. In $§ 5$ we lift these explicit cubic relations to the original invariant ring. Having deduced that the ideal of relations is generated by quadratics and these particular ("small generalized Segre") cubics, we are done with the toric degeneration. Our next goal is to show that the particular cubics lie in the ideal $Q_{L}$ generated by quadratic relations.

In order to take advantage of the $\mathfrak{S}_{L}$-action, in $\$ 6$ we study the tensor powers of the degree one invariants as representations, introducing a useful "partition filtration." Our results will (for example) allow us to write invariants and relations in terms of highly disconnected graphs, which is the key to our later inductive arguments. The last result of this section is disappointing: it is the only place in the article where computer calculation is used. However, the calculations are quite mild - they concern cubic invariants on six points and amount to simple linear algebra problems in vector spaces of dimension at most 35-and we feel that a dedicated human being could perform them in a matter of hours.

In $\S 7$, we show that for $n \geq 10$ all relations are induced from those on fewer points (precisely, $n-2, n-4$ or $n-6$ points), modulo quadratic relations. The cases $n \geq 12$ are direct and structural, but the case $n=10$ is ad hoc and inelegant because "the graphs are too small" to apply the structural techniques. As a consequence, we find that if the ideal is generated by quadratics for $n-2, n-4$ and $n-6$ points then it is for $n$ points as well.

In $\S 8$, we show that the ideal is generated by quadratics when $n \neq 6$. This implies Theorem 1.1 by Remark 1.3. Thanks to the previous section, showing generation by quadratics reduces by induction to showing the result for the three "base cases" where $n$ is 8,10 and 12. We accomplish this by further reducing the 10- and 12-point cases to the 8-point case and then appealing to earlier work for the 8 point case. The reduction from the 10 and 12-point case to the 8-point case is one of the most difficult and least conceptual parts of the paper, so the reader may wish to skip this section on a first reading. One might hope that these results, being finite computations, could be relegated to a computer, but the computations are large enough so that this is not possible with current technology using naive algorithms.

Finally, in $\$ 9$ we show that the quadratic relations are spanned by the simplest binomial relations using the representation theory of $\mathfrak{S}_{L}$, completing the proof of Theorem 1.2.

\subsection{The projective coordinate ring of $X^{n} / / G$}

The third author has observed that many of the formal concepts in this paper-such as outer multiplication and the simple binomial relations-in fact apply to the study of the projective coordinate ring of $X^{n} / / G$ for any projective variety $X$ with an action of a group $G$. He has constructed a formalism for dealing with the resulting structures and formulated a few general finiteness conjectures. One of the more surprising realizations is that the graphical formalism discussed above applies to any variety, in a certain sense. This general point of view may even shed more light on the present case: in this formalism, Theorem 1.2 can be reinterpreted as stating that a certain "ring," made up of the 
rings $R_{1^{n}}$ with varying $n$, is finitely presented. If such finitely presented "rings" were coherent (a weakening of the noetherian property) then one would immediately obtain universal degree bounds on syzygies, as we have for relations. This work will appear in a forthcoming paper.

\subsection{Notation and conventions}

We follow some conventions in an attempt to make the notation less onerous. Throughout, $L$ will be a finite set. By an even set we mean finite set of even cardinality. Semigroups will be in script, e.g., $\mathscr{G}, \mathscr{S}, \mathscr{R}$. Graphs are denoted by uppercase Greek letters, e.g., $\Gamma, \Delta$. Trivalent trees will be denoted by $\Xi$. An edge of a directed (resp. undirected) graph from vertex $x$ to $y$ is denoted $\overrightarrow{x y}$ (resp. $\overline{x y}$ ). In general, $S$ (in various fonts) will refer to constructions involving general directed graphs, and $R$ will refer to regular graphs. We work over $\mathbb{Z}$ (see $§ 1.1)$ in general.

\section{The invariant ring $R_{L}$}

In $\S 2$ we define the ring of invariants $R_{L}$ for a finite set $L$, and give some of its properties.

\subsection{The semigroup $\mathscr{G}_{L}$ and the rings $S_{L}$ and $R_{L}$}

Let $L$ be a finite set. Denote by $\mathscr{G}_{L}$ the set of directed graphs on $L$. Give $\mathscr{G}_{L}$ the structure of a semigroup by defining $\Gamma \cdot \Gamma^{\prime}$ to be the graph on $L$ whose edge set is the disjoint union of the edge sets of $\Gamma$ and $\Gamma^{\prime}$. For an element $\Gamma$ of $\mathscr{G}_{L}$ we denote the corresponding element of the semigroup algebra $\mathbb{Z}\left[\mathscr{G}_{L}\right]$ by $X_{\Gamma}$. (Readers interested in characteristic 0 may freely replace any occurrence of $\mathbb{Z}$ or $\mathbb{Z}[1 / n]$ by $\mathbb{Q}$ throughout.)

For $a, b \in L$ let $\overrightarrow{a b}$ denote the graph in $\mathscr{G}_{L}$ with a single directed edge from $a$ to $b$. Clearly $\mathscr{G}_{L}$ is the free commutative semigroup on the $\overrightarrow{a b}$, and $\mathbb{Z}\left[\mathscr{G}_{L}\right]$ is the polynomial ring on the $X_{\overrightarrow{a b}}$.

Define the ring $S_{L}$ as the quotient of $\mathbb{Z}\left[\mathscr{G}_{L}\right]$ by the following three types of relations, described in $§ 1.2$.

- Loop relation: If $\Gamma$ has a loop then $X_{\Gamma}=0$.

- Sign relation: If $\Gamma$ is obtained from $\Gamma^{\prime}$ by reversing the direction of an edge then $X_{\Gamma}$ $=-X_{\Gamma^{\prime}}$.

- Plücker relation: If $a, b, c$ and $d$ are elements of $L$, then $X_{\overrightarrow{a b}} X_{\overrightarrow{c d}}=X_{\overrightarrow{a d}} X_{\overrightarrow{c b}}+X_{\overrightarrow{a c}} X_{\overrightarrow{b d}}$.

The sign relation implies the loop relation when 2 is inverted. We still write $X_{\Gamma}$ for the image of $X_{\Gamma}$ in the ring $S_{L}$.

Recall that a graph $\Gamma$ on $L$ is said to be regular of degree $d$ if each vertex of $\Gamma$ belongs to precisely $d$ edges. Define $R_{L}$ to be the subgroup of $S_{L}$ generated by the $X_{\Gamma}$ with $\Gamma$ regular. Clearly $R_{L}$ is a subring of $S_{L}$. Grade $R_{L}$ by declaring $X_{\Gamma}$ to be of degree $d$ if $\Gamma$ is regular of degree $d$. If $|L|$ is odd, then every regular graph on $L$ has even degree, so $R_{L}$ is concentrated in even degrees. (The "first and second main theorems of invariant theory," 
mentioned implicitly in $\$ 1.2$, imply that $S_{L}$ is the SL(2)-invariant part of the Cox ring of $\left(\mathbb{P}^{1}\right)^{L}$ and that $R_{L}$ is the SL(2)-invariant part of the projective coordinate ring of $\left(\mathbb{P}^{1}\right)^{L}$ with respect to the line bundle $\mathscr{O}(1)^{\bigotimes L}$.)

These constructions are functorial in $L:$ A map of sets $\phi: L \rightarrow L^{\prime}$ induces a homomorphism $\mathscr{G}_{L} \rightarrow \mathscr{G}_{L^{\prime}}$ of semigroups and thus a homomorphism $\mathbb{Z}\left[\mathscr{G}_{L}\right] \rightarrow \mathbb{Z}\left[\mathscr{G}_{L^{\prime}}\right]$ of semigroup rings. This ring homomorphism respects the sign and Plücker relations and so induces a ring homomorphism $S_{L} \rightarrow S_{L^{\prime}}$. If the fibers of $L$ all have the same cardinality then $R_{L}$ is mapped into $R_{L^{\prime}}$, and we thus obtain a map $R_{L} \rightarrow R_{L^{\prime}}$. As a special case, we see that $\mathfrak{S}_{L}=\operatorname{Aut}(L)$ acts on $R_{L}$.

\subsection{Translation from directed graphs to undirected graphs}

To avoid confusion with signs, it will often be convenient to translate from directed graphs to undirected graphs. Let $L$ be an even set. We denote by $\mathcal{M}_{L}$ the set of directed matchings on $L$. An orientation on $L$ is a map $\epsilon: \mathcal{M}_{L} \rightarrow\{ \pm 1\}$ satisfying $\epsilon(\sigma \Gamma)=\operatorname{sgn}(\sigma) \epsilon(\Gamma)$ for $\sigma \in \mathfrak{S}_{L}$ and $\Gamma \in \mathcal{M}_{L}$. There are two orientations on $L$.

Fix an orientation of $L$. For an undirected matching $\Gamma$ we put $Y_{\Gamma}=\epsilon(\widetilde{\Gamma}) X_{\widetilde{\Gamma}}$ where $\widetilde{\Gamma}$ is any directed matching with underlying undirected matching $\Gamma$. The $Y_{\Gamma}$ span the space $R_{L}^{(1)}$ and satisfy the loop relation and the "undirected Plücker relation"

$$
Y_{\Gamma_{1}}+Y_{\Gamma_{2}}+Y_{\Gamma_{3}}=0
$$

whenever $\Gamma_{2}$ and $\Gamma_{3}$ are obtained by modifying two edges of $\Gamma_{1}$ appropriately. We often prefer to work with the $Y_{\Gamma}$ instead of the $X_{\Gamma}$ since there are no directions to keep track of. However, one must keep in mind that the action of the symmetric group on the $Y_{\Gamma}$ is twisted by the sign character from the most obvious action: $\sigma Y_{\Gamma}=\operatorname{sgn}(\sigma) Y_{\sigma \Gamma}$ for $\sigma \in \mathfrak{S}_{L}$. We often use the $Y_{\Gamma}$ without explicitly mentioning the choice of an orientation.

\subsection{Kempe's generation theorem}

The purpose of this paper is to give a presentation for the ring $R_{L}$. To do this we must first find a set of generators. This problem was solved (for the main case) by Kempe [Ke].

Theorem 2.1 (Kempe). Let $L$ be an even set. Then the ring $R_{L}$ is generated in degree one. Equivalently, the $Y_{\Gamma}\left(\right.$ or $\left.X_{\Gamma}\right)$ with $\Gamma$ a matching generate $R_{L}$.

Proof. We recall the proof of [HMSV1, Theorem 2.3], which is simpler than Kempe's original proof, to motivate later arguments. For any regular graph $\Gamma$ on $L$, we express $Y_{\Gamma}$ as a polynomial in elements of the form $Y_{\Gamma^{\prime}}$ with $\Gamma^{\prime}$ a matching. Partition $L$ arbitrarily into two sets of equal cardinality, one called "positive" and the other "negative." We then have three types of edges: positive (both vertices positive), negative (both negative) and neutral (one positive and one negative). After applying the Plücker relation to a positive and a negative edge, one is left with only neutral edges:

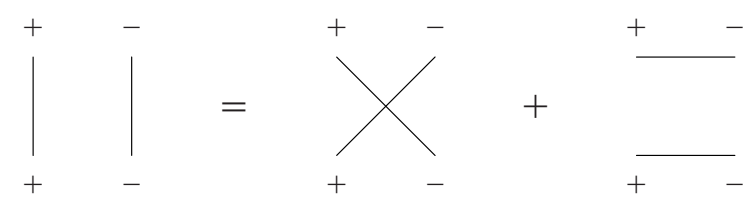


(We have neglected signs in the above identity.) As $\Gamma$ is regular, it has a positive edge if and only if it has a negative one. Thus by repeatedly applying Plücker relations to positive and negative edges we end up with an expression $Y_{\Gamma}=\sum \pm Y_{\Gamma_{i}}$ where the $\Gamma_{i}$ have only neutral edges, and are hence bipartite. Hall's marriage theorem states that in a regular bipartite graph one can find a matching. Thus each $\Gamma_{i}$ can be factored into matchings, which completes the proof.

\subsection{Kempe's basis theorem}

Fix an embedding of $L$ into the unit circle in the plane. We say that a graph $\Gamma$ on $L$ is noncrossing if no two of its edges cross when drawn as chords. The following well-known theorem of Kempe (also from [Ke]) will be used in the proofs of Proposition 3.1 and Lemma 9.3.

Theorem 2.2 (Kempe). The $X_{\Gamma}$ with $\Gamma$ non-crossing span $S_{L}$. The only linear relations among these elements are the sign and loop relations. Thus if one chooses for each undirected loop-free non-crossing graph a direction on the edges then the corresponding $X_{\Gamma}$ form a basis for $S_{L}$. The same is true for $R_{L}$ if one considers regular non-crossing graphs.

In fact, there is a procedure called the straightening algorithm which expresses an arbitrary $X_{\Gamma}$ in terms of the non-crossing basis. The algorithm is simple: take any pair of edges in $\Gamma$ which cross and Plücker them. The algorithm terminates because the total lengths of the edges in each of the two graphs resulting from a Plücker operation is less than that in the original graph. This nearly proves the theorem; for details see [HMSV1, Propositions 2.5, 2.6].

\subsection{Some definitions}

We now define some notation that will be used constantly:

- $R_{L}$ is the ring of invariants, as defined above.

- $V_{L}$ is the first graded piece $R_{L}^{(1)}$ of $R_{L}$; it is spanned by the $Y_{\Gamma}$ with $\Gamma$ a matching.

- $I_{L}$ is the ideal of relations, that is, the kernel of the map $\operatorname{Sym}\left(V_{L}\right) \rightarrow R_{L}$.

- $Q_{L}$ is the ideal of $\operatorname{Sym}\left(V_{L}\right)$ generated by $I_{L}^{(2)}$; it is a subideal of $I_{L}$.

\section{The toric degenerations $\operatorname{gr}_{\Xi} S_{L}$ and $\operatorname{gr}_{\Xi} R_{L}$}

In $\S 3$, we discuss toric degenerations of the rings $S_{L}$ and $R_{L}$. These were first described in [SS], and one was used in [HMSV1]. By a "toric ring" we mean a ring isomorphic to a semigroup algebra, where the semigroup is the set of lattice points in a strictly convex rational polyhedral cone; by a "toric degeneration" of a ring we mean a toric ring obtained as the associated graded of a filtration on the original ring. The main points of $\S 3$ are the following: 
(1) To each trivalent tree $\Xi$ with leaf set $L$ we give a toric degeneration of the rings $S_{L}$ and $R_{L}$, denoted $\operatorname{gr}_{\Xi} S_{L}$ and $\operatorname{gr}_{\Xi} R_{L}$ respectively.

(2) We give a presentation of the $\operatorname{ring} \operatorname{gr}_{\Xi} S_{L}$.

(3) We discuss the theory of weightings on trivalent trees.

(4) We identify the rings $\operatorname{gr}_{\Xi} S_{L}$ and $\operatorname{gr}_{\Xi} R_{L}$ with semigroup algebras of "weightings."

\subsection{Trivalent trees}

By a trivalent tree we mean a connected undirected graph $\Xi$, without cycles, all of whose vertices have valence one or three. We call vertices of valence three trinodes, and vertices of valence one leaves. We say leaves $x$ and $y$ form a matched pair if they share a neighbor (trinode). We say that a trivalent tree is matched if it has more than four vertices and every leaf belongs to a (necessarily unique) matched pair. A matched trivalent tree necessarily has an even number of leaves.

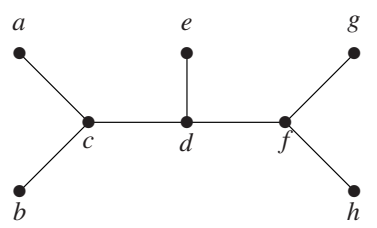

Fig. 1. A trivalent tree. The vertices $c, d$ and $f$ are trinodes; the rest are leaves. The vertices $a$ and $b$ form a matched pair, as do the vertices $g$ and $h$. The tree is not matched because $e$ does not belong to a matched pair.

\subsection{The toric rings $\operatorname{gr}_{\Xi} S_{L}$ and $\operatorname{gr}_{\Xi} R_{L}$}

Let $\Xi$ be a trivalent tree with leaf set $L$. For a graph $\Gamma$ on $L$, define the level of $\Gamma$ (relative to $\Xi)$ as

$$
\left.\operatorname{lev}_{\Xi} \Gamma=\sum_{\overrightarrow{a b}} \text { (the distance from } a \text { to } b \text { in } \Xi\right)
$$

where the sum is over the edges of $\Gamma$, and distance is the number of edges in the "geodesic." Clearly lev $\Xi$ induces a semigroup morphism lev $: \mathscr{G}_{L} \rightarrow \mathbb{Z}_{\geq 0}$. Define an increasing filtration $F_{\Xi}$ on $\mathbb{Z}\left[\mathscr{G}_{L}\right]$ by letting $F_{\Xi}^{i} \mathbb{Z}\left[\mathscr{G}_{L}\right]$ be the subspace of $\mathbb{Z}\left[\mathscr{G}_{L}\right]$ spanned by the $X_{\Gamma}$ with lev $\Xi \Gamma \leq i$. (The notation lev $\Xi$ will not be used further.) Let $F_{\Xi}^{i} S_{L}$ be the image of $F_{\Xi}^{i} \mathbb{Z}\left[\mathscr{G}_{L}\right]$ under the surjection $\mathbb{Z}\left[\mathscr{G}_{L}\right] \rightarrow S_{L}$, giving a filtration of the ring $S_{L}$. Let $\operatorname{gr}_{\Xi} S_{L}$ denote the associated graded ring. We will show that $\operatorname{gr}_{\Xi} S_{L}$ is a toric ring (Proposition 3.3). For a graph $\Gamma$ of level $n$, let $\bar{X}_{\Gamma}$ denote the image of $X_{\Gamma}$ in $F_{\Xi}^{n} S_{L} / F_{\Xi}^{n-1} S_{L}$. Clearly the $\bar{X}_{\Gamma}$ span $\operatorname{gr}_{\Xi} S_{L}$.

Let $F_{\Xi}^{i} R_{L}$ be the filtration on $R_{L}$ induced from its inclusion into $S_{L}$. Let $\operatorname{gr}_{\Xi} R_{L}$ be the associated graded ring. It is naturally the subring of $\operatorname{gr}_{\Xi} S_{L}$ spanned by the $\bar{X}_{\Gamma}$ for which $\Gamma$ is regular. 


\subsection{Presentation of the ring $\operatorname{gr}_{\Xi} S_{L}$}

Let $J_{\Xi}$ denote the ideal in $\mathbb{Z}\left[\mathscr{G}_{L}\right]$ generated by the following (cf. $\S 2.1$ ):

- Loop relation: If $\Gamma$ has a loop then $X_{\Gamma}=0$.

- Sign relation: If $\Gamma^{\prime}$ is obtained from $\Gamma$ by reversing the direction of an edge then $X_{\Gamma}=$ $-X_{\Gamma^{\prime}}$

- Toric Plücker relation: If $a, b, c$ and $d$ are elements of $L$ satisfying:

the path from $a$ to $b$ in $\Xi$ meets the path from $c$ to $d$, and the path from $a$ to $c$ meets the path from $b$ to $d$ (see Figure 2)

then $X_{\overrightarrow{a b}} X_{\overrightarrow{c d}}=X_{\overrightarrow{a c}} X_{\overrightarrow{b d}}$.

(The notation $J_{\Xi}$ is only used in §3.3.)

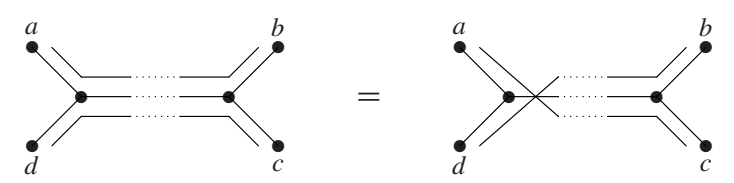

Fig. 2. The toric Plücker relation $X_{\overrightarrow{a b}} X_{\overrightarrow{c d}}=X_{\overrightarrow{a c}} X_{\overrightarrow{b d}}$. Both pairs of geodesics overlap on the horizontal edge of the trivalent tree $\Xi$. Note that $\overrightarrow{a d}$ and $\overrightarrow{b c}$ do not overlap.

The purpose of this section is to prove the following, used in $\S 3.5$ to identify $\operatorname{gr}_{\Xi} S_{L}$ with a semigroup algebra of "weightings."

Proposition 3.1. The map $\mathbb{Z}\left[\mathscr{G}_{L}\right] \rightarrow \operatorname{gr}_{\Xi} S_{L}$ given by $X_{\Gamma} \mapsto \bar{X}_{\Gamma}$ is surjective with kernel $J_{\Xi}$.

The kernel $I$ of the map $\mathbb{Z}\left[\mathscr{G}_{L}\right] \rightarrow S_{L}$ is generated by the sign and Plücker relations. The kernel of the map $\mathbb{Z}\left[\mathscr{G}_{L}\right] \rightarrow \operatorname{gr}_{\Xi} S_{L}$ is the ideal generated by the leading terms of all elements of $I$. In general, of course, this is not the same as the ideal generated by the leading terms of a generating set of $I$. Proposition 3.1 says that in this situation, however, this is the case.

Proof of Proposition 3.1. The map is clearly surjective and contains the sign relation in its kernel. We now check that the toric Plücker relation lies in its kernel as well. Let $a, b$, $c$ and $d$ belong to $L$ and satisfy (3.1). The equation

$$
X_{\overrightarrow{a b}} X_{\overrightarrow{c d}}=X_{\overrightarrow{a c}} X_{\overrightarrow{b d}}+X_{\overrightarrow{a d}} X_{\overrightarrow{b c}}
$$

holds in $S_{L}$ (the normal Plücker relation). The two graphs $\overrightarrow{a b} \cdot \overrightarrow{c d}$ and $\overrightarrow{a c} \cdot \overrightarrow{b d}$ have the same level, say $n$, since when drawn in $\Xi$ they use the same edges with the same multiplicity (see Figure 2). The remaining graph $\overrightarrow{a d} \cdot \overrightarrow{b c}$ has level less than $n$ (again, see Figure 2). Thus all terms in the above relation lie in $F_{\Xi}^{n} S_{L}$. Reducing modulo $F_{\Xi}^{n-1} S_{L}$ we obtain

$$
\bar{X}_{\overrightarrow{a b}} \bar{X}_{\overrightarrow{c d}}=\bar{X}_{\overrightarrow{a c}} \bar{X}_{\overrightarrow{b d}},
$$

which shows that the toric Plücker relation lies in $J_{\Xi}$. 
We now show that non-crossing graphs span $\mathbb{Z}\left[\mathscr{G}_{L}\right] / J_{\Xi}$. Embed $L$ into the unit circle in the plane in such a way that $\Xi$ can be drawn inside the circle without any crossings. Let $\Gamma$ be a graph on $L$ which contains crossing edges $\overrightarrow{a b}$ and $\overrightarrow{c d}$. The paths $a b$ and $c d$ then meet in $\Xi$. The same reasoning as in the non-toric case now applies: applying the toric Plücker relation to this pair of edges yields an identity $\bar{X}_{\Gamma}=\bar{X}_{\Gamma^{\prime}}$ where the total length of edges in $\Gamma^{\prime}$ is less than that of $\Gamma$ (here length is computed as distance in the plane, not the trivalent tree). Continuing in this manner, we get an expression $\bar{X}_{\Gamma}=\bar{X}_{\Gamma^{\prime}}$ where $\Gamma^{\prime}$ is non-crossing.

It is now formal to conclude that $\mathbb{Z}\left[\mathscr{G}_{L}\right] / J_{\Xi} \rightarrow \operatorname{gr}_{\Xi} S_{L}$ is an isomorphism. We elaborate on this. Choose a set $Z$ of directed non-crossing graphs such that for each undirected non-crossing graph $\Gamma$ there is a unique way to direct the edges of $\Gamma$ so that the resulting graph belongs to $Z$. The previous paragraph shows that if $\Gamma$ is any graph of level $n$, we can find $\Gamma^{\prime} \in Z$ such that $X_{\Gamma}= \pm X_{\Gamma^{\prime}}+Y$ holds in $S_{L}$, where $Y \in F_{\Xi}^{n-1} S_{L}$. Applying this result to $Y$ repeatedly, we find that the $X_{\Gamma}$ with $\Gamma$ in $Z$ and level at most $n$ form a basis of $F_{\Xi}^{n} S_{L}$ (we already know they are linearly independent). It thus follows that the $\bar{X}_{\Gamma}$ with $\Gamma$ in $Z$ are linearly independent in $\operatorname{gr}_{\Xi} S_{L}$. Since the surjection $\mathbb{Z}\left[\mathscr{G}_{L}\right] / J_{\Xi} \rightarrow \operatorname{gr}_{\Xi} S_{L}$ takes a spanning set to a set of linearly independent vectors, it must be an isomorphism.

\subsection{Weightings}

A weighting $\xi$ on a trivalent tree $\Xi$ is an assignment of a non-negative integer to each edge of $\Xi$. Define the weight triple of a trinode $v$ of $\Xi$ to be the weights of the three edges connected to $v$. (We write it as an ordered triple even though it is not ordered.) Consider the following two equivalent conditions on weight triples $(a, b, c)$ :

(W1) The triple $(a, b, c)$ satisfies the triangle inequalities ( $a, b$ and $c$ can form the sides of a triangle) and $a+b+c$ is even.

(W2) There exists a triple $(x, y, z)$ of non-negative integers such that $a=x+y, b=x+z$ and $c=y+z$.

Note that the triple $(x, y, z)$ is uniquely determined by $(a, b, c)$. We say that a weighting is admissible if the weight triple at each trinode satisfies these conditions.

Let $\Gamma$ be an undirected graph on the leaves of $\Xi$. We say that an edge $e$ of $\Xi$ meets an edge $\overline{i j}$ of $\Gamma$ if $e$ occurs in the geodesic joining $i$ and $j$. We define a weighting $\xi_{\Gamma}$ of $\Xi$ by assigning to an edge of $\Xi$ the number of edges of $\Gamma$ which it meets. We call $\xi_{\Gamma}$ the weighting of $\Xi$ associated to $\Gamma$ (see Figure 3).

Define (toric) Plücker equivalence to be the equivalence relation $\sim$ on the semigroup of undirected graphs $\mathscr{G}_{L}^{\text {un }}$ on $L$ generated by the following two conditions:

- Given $a, b, c, d$ in $L$ satisfying (3.1), $\overline{a b} \cdot \overline{c d} \sim \overline{a c} \cdot \overline{b d}$.

- If $\Gamma \sim \Gamma^{\prime}$ and $\Gamma^{\prime \prime}$ is any graph then $\Gamma \cdot \Gamma^{\prime \prime} \sim \Gamma^{\prime} \cdot \Gamma^{\prime \prime}$.

The following result gives a correspondence between graphs and weightings.

Proposition 3.2. Associating a weighting of $\Xi$ to a graph induces a bijection between the Plücker classes of undirected graphs on the leaves of $\Xi$ and the admissible weightings of $\Xi$. 


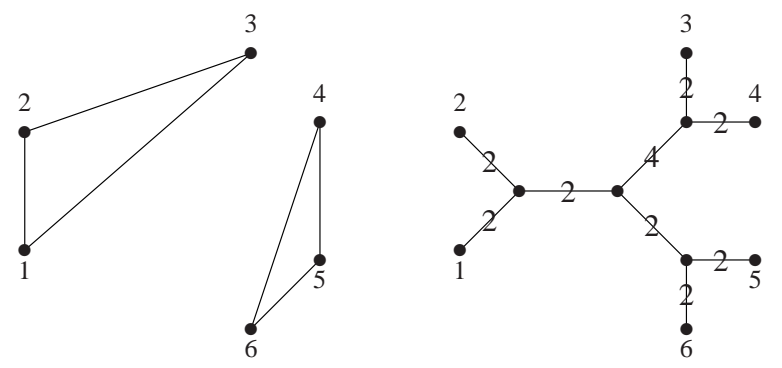

Fig. 3. A graph on the leaves of a trivalent tree and its associated weighting.

Proof. Let $\Gamma$ be a graph and let $\xi$ be its associated weighting. We first show that $\xi$ is admissible. Let $v$ be a trinode of $\Xi$ and let $(a, b, c)$ be its weight triple. Consider the picture

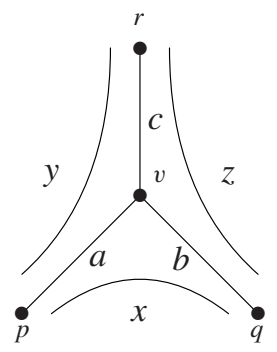

Here $a$ is the number of edges of $\Gamma$ that meet edge $\overline{p v} ; b$ and $c$ are defined similarly. We let $x$ be the number of edges of $\Gamma$ which, when drawn as geodesics on $\Xi$, go through both $p$ and $q$; we let $y$ and $z$ be the analogous quantities. Clearly $a=x+y, b=x+z$, and $c=y+z$. Thus we have shown that the weighting $\xi$ satisfies condition (W2) at each trinode and is therefore admissible.

If we apply a toric Plücker relation to $\Gamma$ then its associated weighting does not change: the two pairs of edges in the toric Plücker relation contain the same edges when drawn as geodesics. Thus associating a weighting to a graph yields a well-defined map from the Plücker classes of graphs to the set of admissible weightings. We now show that this map is bijective.

We first prove that it is surjective. We are given an admissible weighting $\xi$ and we must produce a graph $\Gamma$ such that $\xi$ is its associated weighting. To do this it suffices to prove the following: given an admissible weighting $\xi$ there exist two leaves $i$ and $j$ of $\Xi$ such that when the geodesic joining $i$ and $j$ is subtracted from $\xi$, the resulting weighting is still admissible. For, if this is the case, then we can let $\overline{i j}$ be an edge of $\Gamma$, subtract the geodesic joining $i$ and $j$ from $\xi$ and proceed by induction.

Thus let $\xi$ be an admissible weighting of $\Xi$. Let $i$ be any leaf of $\Xi$ for which $\xi$ does not vanish on the edge containing $i$. We produce the vertex $j$ by the following greedy algorithm. Put $v_{0}=i$ and let $v_{1}$ be the unique trinode connected to $i$. Assume now that we have defined $v_{0}$ through $v_{k}$. If $v_{k}$ is a leaf then stop and put $j=v_{k}$. Otherwise $v_{k}$ is connected to two vertices other than $v_{k-1}$. Let $v_{k+1}$ be the one for which the corresponding edge has higher weight; if the two edges have the same weight then pick $v_{k+1}$ 
arbitrarily. We have thus produced a pair of leaves $i$ and $j$; note that the $v_{k}$ are the trinodes in a geodesic joining $i$ and $j$. We must show that when this geodesic is subtracted from $\xi$, the resulting weighting is still admissible.

Let $\ell$ lie strictly between 0 and $k$ so that $v_{\ell}$ is a trinode. We have the picture

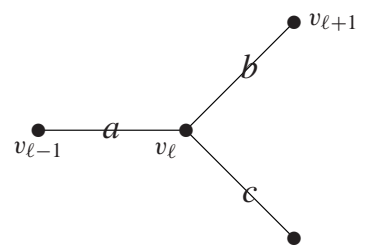

By definition of $v_{\ell+1}$ we have $b \geq c$. We know that $(a, b, c)$ satisfies (W1). Clearly $(a-1, b-1, c)$ still satisfies the parity condition. We must show that it still satisfies the triangle inequalities, which amounts to proving $c \leq a+b-2$. This inequality could fail in two ways: 1) $a=0$ and $c$ equals $b$ or $b-1$; or 2) $a=1$ and $b=c$. The first case is ruled out by the way we selected $v_{\ell-1}$ and an easy induction argument. The second case is ruled out since $a+b+c$ is even. This proves that the greedy algorithm indeed works and completes the proof that our map is surjective. Note that in building the graph from the weighting there are many arbitrary choices.

We now prove that the map is injective, i.e., if $\Gamma$ and $\Gamma^{\prime}$ have the same weighting $\xi$ then they are Plücker equivalent. To show this we show that $\Gamma$ is Plücker equivalent to any of the graphs constructed out of $\xi$ by using the greedy algorithm. It suffices to prove that if $v_{0}, \ldots, v_{k}$ is a sequence coming out of the greedy algorithm then we can apply toric Plücker relations to $\Gamma$ so that $v_{0}$ and $v_{k}$ are connected in $\Gamma$. For then we may remove this edge from $\Gamma$ and the corresponding path in $\xi$ and proceed by induction.

Thus let $v_{0}, \ldots, v_{k}$ come out of the greedy algorithm. We prove by induction on $\ell$ that $\Gamma$ is Plücker equivalent to a graph containing an edge which passes through $v_{0}$ and $v_{\ell}$ (we say that an edge of $\Gamma$ passes through two vertices of $\Xi$ if its corresponding geodesic does). This is clear for $\ell=1$. Thus assume it is true for $\ell$ and we show that it is true for $\ell+1$. We have the picture

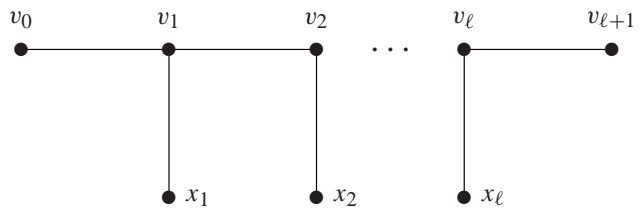

Here $x_{i}$ is the unique vertex connected to $v_{i}$ besides $v_{i-1}$ and $v_{i+1}$. We have assumed that $\Gamma$ contains an edge $e$ passing through $v_{0}$ and $v_{\ell}$; we must show that $\Gamma$ is Plücker equivalent to a graph containing an edge passing through $v_{0}$ and $v_{\ell+1}$. Now, $e$ itself passes through either $v_{\ell+1}$ or $x_{\ell}$. In the former case we are done. Thus we may assume that $e$ passes through $x_{\ell}$.

By the definition of the greedy algorithm we have

$$
\xi\left(\overline{v_{\ell} x_{\ell}}\right) \leq \xi\left(\overline{v_{\ell} v_{\ell+1}}\right), \quad \xi\left(\overline{v_{i} v_{i+1}}\right) \neq 0 .
$$


Thus there exists an edge of $\Gamma$ passing through $v_{\ell}$ and $v_{\ell+1}$. If every edge which passed through $v_{\ell}$ and $v_{\ell+1}$ also passed through $x_{\ell}$ then, by the inequality, every edge which passed through $x_{\ell}$ and $v_{\ell}$ would also pass through $v_{\ell+1}$; it would follow that no edge could pass through $v_{\ell-1}$ and $v_{\ell}$. However, this would imply $\xi\left(\overline{v_{\ell-1} v_{\ell}}\right)=0$, a contradiction. Thus there exists an edge $e^{\prime}$ of $\Gamma$ which passes through $v_{\ell+1}$ and $v_{\ell}$ but not through $x_{\ell}$. If $v_{0}$ is a vertex of $e^{\prime}$ then we are done. Otherwise, applying the toric Plücker relation to $e$ and $e^{\prime}$ yields a graph containing an edge passing through $v_{0}$ and $v_{\ell+1}$. This completes the proof.

\subsection{The rings $\operatorname{gr}_{\Xi} S_{L}$ and $\operatorname{gr}_{\Xi} R_{L}$ as semigroup algebras of weightings}

Let $\Xi$ be a trivalent tree with leaf set $L$. Embed $L$ into the unit circle in such a way that $\Xi$ can be drawn inside the unit circle without crossings. Choose a total order on $L$ which is compatible with its embedding into the circle in the sense that if $a \leq b \leq c$ then one encounters $b$ when traveling clockwise from $a$ to $c$. For $a, b \in L$ define $\epsilon_{a b}$ to be 1 if $a<b,-1$ if $a>b$, and 0 if $a=b$. For a directed graph $\Gamma$ on $L$ define $\epsilon_{\Gamma}$ to be the product of the $\epsilon_{a b}$ over the edges $\overrightarrow{a b}$ of $\Gamma$. We write $\Gamma^{\text {un }}$ for the undirected graph associated to $\Gamma$. Finally, let $\mathscr{S}_{\Xi}$ denote the set of admissible weights on $\Xi$. It is a semigroup since the sum of two admissible weightings is again admissible. We can now prove:

Proposition 3.3. There is a unique isomorphism of rings $\operatorname{gr}_{\Xi} S_{L} \rightarrow \mathbb{Z}\left[\mathscr{S}_{\Xi}\right]$ mapping $\bar{X}_{\Gamma}$ to $\epsilon_{\Gamma} \xi_{\Gamma}$ un.

Proof. We define an auxiliary ring by modifying the sign relation in the presentation of $\operatorname{gr}_{\Xi} S_{L}$ given in $\S 3.3$. Define the ideal $J_{\Xi}^{\text {un }}$ of $\mathbb{Z}\left[\mathscr{G}_{L}\right]$ by the following types of relations:

- Loop relation: If $\Gamma$ has a loop then $X_{\Gamma}=0$.

- Modified sign relation: If $\Gamma^{\prime}$ is obtained from $\Gamma$ by reversing the direction of an edge then $X_{\Gamma}=X_{\Gamma^{\prime}}$.

- Toric Plücker relation: As in $\$ 3.3$.

Denote the image of $X_{\Gamma}$ in $\mathbb{Z}[\mathscr{G}] / J_{L}^{\text {un }}$ by $\bar{X}_{\Gamma}^{\text {un }}$. Note that $\bar{X}_{\Gamma}^{\text {un }}$ makes sense for an undirected graph $\Gamma$, and that if $\Gamma$ and $\Gamma^{\prime}$ are Plücker equivalent undirected graphs then the toric Plücker relation implies $\bar{X}_{\Gamma}^{\text {un }}=\bar{X}_{\Gamma^{\prime}}^{\text {un }}$. By comparing the definition of $J_{L}^{\text {un }}$ to the presentation of $\operatorname{gr}_{\Xi} S_{L}$ given in Proposition 3.1, we find that the map

$$
\operatorname{gr}_{\Xi} S_{L}=\mathbb{Z}\left[\mathscr{G}_{L}\right] / J_{\Xi} \rightarrow \mathbb{Z}\left[\mathscr{G}_{L}\right] / J_{\Xi}^{\text {un }}, \quad \bar{X}_{\Gamma} \mapsto \epsilon_{\Gamma} \bar{X}_{\Gamma}^{\text {un }},
$$

is well-defined and an isomorphism. From the equivalence of weightings and Plücker classes of undirected graphs given in Proposition 3.2, we find that the map

$$
\mathbb{Z}\left[\mathscr{G}_{L}\right] / J_{\Xi}^{\text {un }} \rightarrow \mathbb{Z}\left[\mathscr{S}_{\Xi}\right], \quad \bar{X}_{\Gamma}^{\text {un }} \mapsto \xi_{\Gamma \text { un }},
$$

is a well-defined isomorphism. The proposition now follows.

We translate this result to the regular case. Call a weighting $\xi$ on $\Xi$ regular of degree $d$ if for each leaf $v$ we have $\xi\left(e_{v}\right)=d$, where $e_{v}$ is the unique edge containing $v$. Let $\mathscr{R}_{\Xi}$ be the semigroup of admissible regular weightings on $\Xi$. We then have: 
Corollary 3.4. There is a unique isomorphism of graded rings $\operatorname{gr}_{\Xi} R_{L} \rightarrow \mathbb{Z}\left[\mathscr{R}_{\Xi}\right]$ which takes $\bar{X}_{\Gamma}$ to $\epsilon_{\Gamma} \cdot \xi_{\Gamma \text { un. }}$

Proposition 3.3 and Corollary 3.4 show that $\operatorname{gr}_{\Xi} S_{L}$ and $\operatorname{gr}_{\Xi} R_{L}$ are semigroup algebras and therefore toric rings. (It is not difficult to see that $\mathscr{S}_{\Xi}$ and $\mathscr{R} \Xi$ are the set of lattice points in a strictly convex rational polyhedral cone, but this will not be of importance to us.)

\subsection{Reduced weightings}

We close $\S 3$ with a discussion of reduced weightings, which we use in $\S 4-5$. Let $\Xi$ be a trivalent tree. By a reduced weighting on $\Xi$ we simply mean a weighting on $\Xi$-the terms are synonymous but used to distinguish the usage of "admissible." We say that a reduced weighting is admissible if it satisfies the triangle inequality (as in (W1)) at each trinode; the parity condition is not enforced. We say that a reduced weighting $\xi$ is regular of degree $\leq d$ if $\xi\left(e_{v}\right) \leq d$ for all leaves $v$, where $e_{v}$ is the edge meeting leaf $v$. Let $\overline{\mathscr{R}}_{\Xi}$ be the set of all ordered pairs $(\xi, d)$ with $d$ a non-negative integer and $\xi$ an admissible reduced weighting which is regular of degree $\leq d$. We define the degree of $(\xi, d)$ to be $d$.

Let $\Xi$ be a matched trivalent tree and let $\Xi^{-}$be the trivalent tree obtained by deleting the leaves of $\Xi$ and the edges that they touch. We call $\Xi^{-}$the truncation of $\Xi$.

Proposition 3.5. With notation as above, there is a canonical isomorphism of semigroups $\mathscr{R}_{\Xi} \rightarrow \overline{\mathscr{R}}_{\Xi^{-}}$preserving degree. The image of a weighting $\xi$ on $\Xi$ is the pair $\left(\xi^{\prime}, d\right)$ where $d$ is the degree of $\xi$ and $\xi^{\prime}$ is the weighting on $\Xi^{-}$given by $\xi^{\prime}(x)=\frac{1}{2} \xi(x)$.

The proof is easy. See Figure 4 for an illustration.
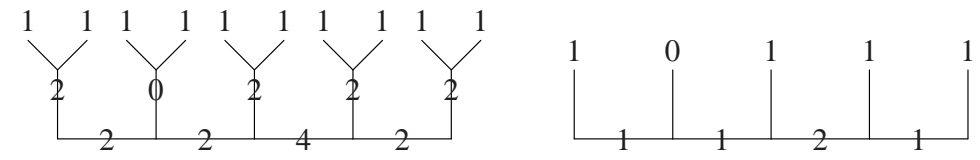

Fig. 4. An illustration of Proposition 3.5. The admissible weighting $\xi$ on the left is regular of degree 1 , the associated reduced admissible weighting $\xi^{-}$on the right is regular of degree $\leq 1$.

\section{The toric ideal is generated by quadratics and toric generalized Segre cubics}

We have described a family of toric degenerations of $R_{L}$, depending on a choice of trivalent tree. The purpose of $\S 4$ is to choose a specific family of trees (the Y-trees) to ensure the degenerated ring (i) is generated in degree one, (ii) has relations generated in degrees two and three, and (iii) is such that there is a precise description of the degree three relations:

Theorem 4.1. Let $\Xi$ be a Y-tree (defined in §4.1). Then $\mathrm{gr}_{\Xi} R_{L}$ is generated in degree one, and the relations between degree one elements are generated by quadratic relations and the generalized toric Segre cubic relations (defined in §4.4). 
(Manon $[\mathrm{M}]$ independently solved the presentation problem for a large class of weighted trivalent trees, including this one; however, we will need the form of (iii).)

\subsection{The $Y$ - and caterpillar trees}

For $r \geq 3$, define the $r$ th $Y$-tree as:

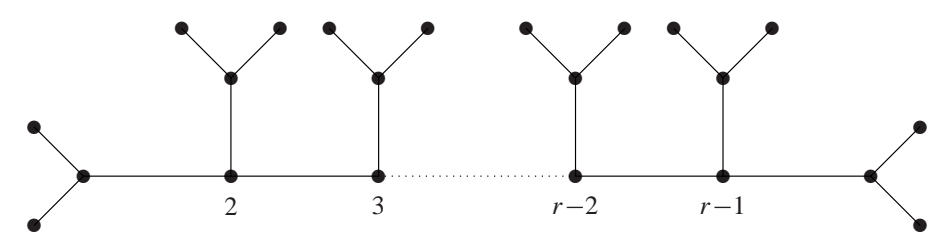

There are $r$ "Y's" in the tree, and $2 r$ leaves. We call the vertex at $i$ the $i$ th base vertex. We call an edge between two base vertices a base edge. By a stalk we mean one of the internal edges in one of the Y's. By the $i$ th stalk, for $2 \leq i \leq r-1$, we mean the one above the $i$ th base vertex. We call the remaining two stalks at either end of the tree the 1 st and $r$ th stalk. The Y-tree is a matched tree (\$3.1). Sometimes we bend the first and last horizontal edges so that all the Y's are in a row, as in Figure 4. The $r$ th caterpillar tree is the truncation of the $r$ th Y-tree:

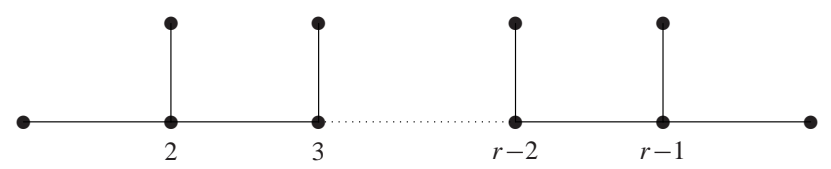

We use the same terminology (base edges, stalks, etc.) for caterpillar trees. Note that the third caterpillar tree is just a trinode.

\subsection{Generators of $\operatorname{gr}_{\Xi} R_{L}$}

We now prove the first half of Theorem 4.1.

Proposition 4.2. Let $\Xi$ be a $Y$-tree. Then the ring $\mathrm{gr}_{\Xi} R_{L}$ is generated in degree one.

Proof. (This proof essentially applies to any matched tree.) We follow the spirit of our proof of Kempe's theorem (Theorem 2.1). Assign to each leaf of $\Xi$ a sign in such a way that in each matched pair of leaves one leaf is positive and the other is negative. Suppose $\Gamma$ is a regular undirected graph of degree $k$. Each edge of $\Gamma$ is then either positive, negative or neutral. We will show that $\Gamma$ is Plücker equivalent (\$3.4) to a graph with only neutral edges. As in the proof of Theorem 2.1, this implies that it is a product of matchings, expressing $\bar{X}_{\Gamma}$ as a product of degree one elements. This proof is more difficult than that of Theorem 2.1 because we are now only allowed to apply the Plücker relation to pairs of edges of $\Gamma$ which meet (i.e., whose geodesics meet) in the tree $\Xi$.

Step 1: Given a positive and negative edge of $\Gamma$ which meet in $\Xi$, apply the toric Plücker relation. In the resulting graph the two new edges are both neutral and so the number of non-neutral edges has decreased. We can thus continue this process until we 
have reached a graph which is Plücker equivalent to $\Gamma$ and contains no overlapping positive and negative edges.

Step 2: Suppose there are still non-neutral paths, but no two of opposite parity meet each other. Let $e$ be the leftmost base edge of $\Xi$ which separates non-neutral edges of $\Gamma$ of opposite parity and let $e^{\prime}$ be the edge to the left of $e$. The edge $e^{\prime}$ will be a base edge unless $e$ is the first base edge, in which case it will be the first stalk. All non-neutral edges of $\Gamma$ which are entirely to the left of $e$ are of the same type, say positive. All edges of $\Gamma$ which pass through $e$ are neutral.

There is a positive edge $\overline{a b}$ of $\Gamma$ which contains $e^{\prime}$. We claim there is an edge $\overline{c d}$ of $\Gamma$ which contains $e$ and where $d$ is positive and to the right of $e$. Assuming the claim (which we prove below), $\overline{a b}$ and $\overline{c d}$ must meet (either at $e^{\prime}$ or at the stalk between $e^{\prime}$ and $e$ ); now apply the toric Plücker relation to this pair. The resulting positive path contains $e$. This brings the leftmost cluster of positive paths closer to the negative paths. By continuing this process, we will eventually cause the two clusters to meet, at which point we return to Step 1 and reduce the number of non-neutral edges. Continuing in this manner, we will eventually remove all non-neutral edges.

Proof of claim: We now prove the claim that there must exist an edge $\overline{c d}$ of $\Gamma$ containing $e$ and for which $d$ is positive and to the right of $e$. Suppose there are $2 m$ leaves to the left of $e$. Let $A$ (resp. $B, C$ ) be the number of positive (resp. negative, neutral) edges of $\Gamma$ entirely to the left of $e$. Let $D$ (resp. $E$ ) be the number of neutral edges containing $e$ for which the positive (resp. negative) leaf is to the left of $e$. Then

$$
2 A+C+D=m k, \quad 2 B+C+E=m k
$$

as the first (resp. second) counts $k$ times each positive (resp. negative) leaf to the left of $e$, and there are $m$ such leaves. We thus find $2 A+D=2 B+E$ and as $B=0$ and $A \neq 0$ by assumption we conclude $E \neq 0$, as claimed.

\subsection{Relations in a semigroup algebra}

We now turn our attention to relations in $\operatorname{gr}_{\Xi} R_{L}$. We begin with a general discussion of relations in a semigroup algebra. Let $\mathscr{R}$ be a semigroup equipped with a homomorphism $\operatorname{deg}: \mathscr{R} \rightarrow \mathbb{Z}_{\geq 0}$, so that the semigroup algebra $\mathbb{Z}[\mathscr{R}]$ is graded. Assume that $\mathscr{R}$ is generated by $\mathscr{V}=\operatorname{deg}^{-1}(1)$, so that the natural map $\operatorname{Sym}(\mathbb{Z}\langle\mathscr{V}\rangle) \rightarrow \mathbb{Z}$ is a surjection (here $\operatorname{Sym}(\mathbb{Z}\langle\mathscr{V}\rangle)$ is the polynomial ring in indeterminates $\mathscr{V}$ while $\mathbb{Z}[\mathscr{R}]$ is the semigroup algebra of $\mathscr{R}$ ). Call the kernel $I$ of this surjection the ideal of relations of $\mathbb{Z}[\mathscr{R}]$.

Let $\xi=\left(\xi_{1}, \ldots, \xi_{n}\right)$ and $\underline{\xi}^{\prime}=\left(\xi_{1}^{\prime}, \ldots, \xi_{n}^{\prime}\right)$ be two elements of $\mathscr{V}^{n}$ such that $\sum \xi_{i}=$ $\sum \xi_{i}^{\prime}$. We then write $\underline{\xi} \sim \underline{\xi}^{\prime}$ and say that $\underline{\xi}$ and $\underline{\xi}^{\prime}$ are related. For an element $\xi$ of $\mathscr{V}$ let $[\xi]$ denote the corresponding element of $\operatorname{Sym}(\overline{\mathbb{Z}}\langle\mathscr{V}\rangle)$; for an element $\underline{\xi}$ of $\mathscr{V}^{n}$ let $[\underline{\xi}]$ denote the monomial $\left[\xi_{1}\right] \cdots\left[\xi_{n}\right]$. Given related $\underline{\xi}$ and $\xi^{\prime}$ the element $[\underline{\xi}]-\left[\xi^{\prime}\right]$ belongs to $I$. We call such relations binomial relations. One easily verifies that $\bar{I}$ is generated by binomial relations.

We say that a relation $\underline{\xi} \sim \underline{\xi}^{\prime}$ has degree $\leq k$ if $\xi_{i}=\xi_{i}^{\prime}$ holds for all but $k$ indices. We say that $\underline{\xi} \sim \xi^{\prime}$ has essentially $\overline{\text { degree }} \leq k$ if there exists a sequence of relations

$$
\underline{\xi}=\underline{\xi}^{(0)} \sim \underline{\xi}^{(1)} \sim \cdots \sim \underline{\xi}^{(p)}=\underline{\xi}^{\prime}
$$


for which each $\underline{\xi}^{(i)} \sim \underline{\xi}^{(i+1)}$ has degree $\leq k$. This notation is only relevant to us when $k$ is 2 or 3 and we then use the terms "essentially quadratic" and "essentially cubic." If $\underline{\xi} \sim \xi^{\prime}$ has essential degree $\leq k$ then the element $[\underline{\xi}]-\left[\xi^{\prime}\right]$ of $I$ lies in the ideal generated by the $k$ th graded piece of $I$.

Note that we consider our tuples as ordered, so that $\left(\xi_{1}, \xi_{2}\right)=\left(\xi_{2}, \xi_{1}\right)$ constitutes a non-trivial relation. However, as the symmetric group is generated by transpositions, it follows that if $\underline{\xi}^{\prime}$ is a permutation of the tuple $\underline{\xi}$ then the relation $\underline{\xi} \sim \underline{\xi}^{\prime}$ is essentially quadratic.

\subsection{The toric generalized Segre cubic relation}

We have the following relation between degree one reduced weightings on the third caterpillar tree:

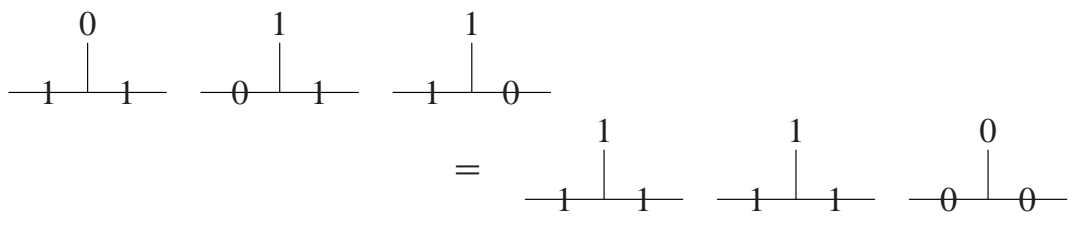

(We will omit + signs in such equations, interpreting them as binomial relations in $\mathbb{Z}[\mathscr{R}]$.) One obtains this relation by converting the usual graphical Segre cubic (1.2) into a relation between weightings on the third Y-tree and then passing to the associated reduced weighting on the third caterpillar tree. One may verify by hand that this single relation generates all relations among admissible reduced weightings on the third caterpillar tree.

We now introduce a class of toric relations that generalize (4.1). We call a reduced weighting of $\Xi^{-}$of degree $\leq 1$ a reduced matching. Recall that the set $\mathscr{V} \Xi$ of reduced matchings generates $\mathscr{R}_{\Xi}$ (Proposition 4.2). Let $X, Y$ and $Z$ be reduced matchings on the $r$ th caterpillar tree such that $X$ and $Y$ take value 1 on the $r$ th stalk and $Z$ takes value 0 on the $r$ th stalk. Let $X^{\prime}, Y^{\prime}$ and $Z^{\prime}$ be reduced matchings on the $s$ th caterpillar tree such that $X^{\prime}$ and $Y^{\prime}$ take value 1 on the first stalk and $Z^{\prime}$ takes value 0 on the first stalk. We then have the relation

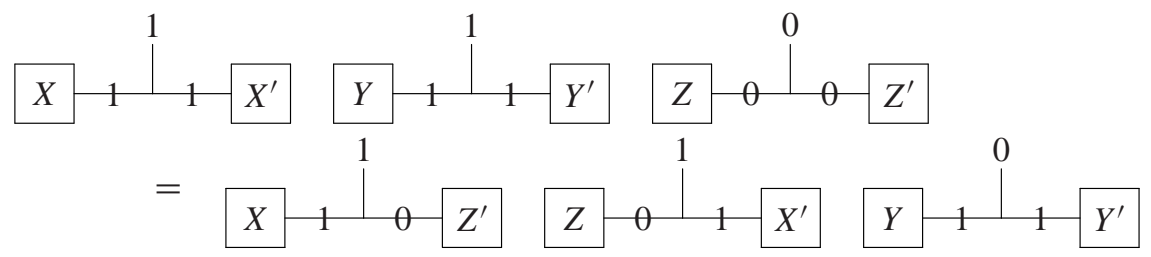

We call these toric generalized Segre cubic relations.

\subsection{The type vector of a triple}

Let $\underline{\xi}=\left(\xi_{1}, \xi_{2}, \xi_{3}\right)$ be a triple of reduced matchings on the $r$ th caterpillar tree. Define the type of $\xi$ at the $i$ th base vertex to be one of $A, B$ or $\emptyset$, as follows. We call $\underline{\xi}$ type $A$ at $i$ if 
it looks like

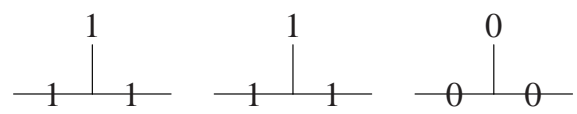

at the $i$ th vertex (the order of the triple is irrelevant). We call $\underline{\xi}$ type $B$ at $i$ if it looks like

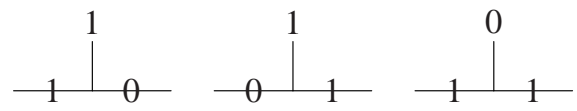

there. In all other cases we call $\xi$ type $\emptyset$ at $i$. We define the type vector of $\xi$, denoted $t(\xi)$, to be the ordered tuple of the $r-2$ types of $\xi$. The type of a triple is a quadratic invariant-if $\underline{\xi} \sim \underline{\xi}^{\prime}$ is an essentially quadratic relation between triples, then $t(\underline{\xi})=t\left(\underline{\xi}^{\prime}\right)$. The toric generalized Segre cubic changes the type.

\subsection{Reformulation of Theorem 4.1}

Rather than proving the statement about relations in Theorem 4.1 directly, we will prove the following:

Proposition 4.3. Let $\Xi$ be the rth caterpillar tree $(r \geq 3)$. Then the ideal of relations $I_{\Xi}$ of $\operatorname{gr}_{\Xi} R_{L}=\mathbb{Z}\left[\mathscr{R}_{\Xi}\right]$ is generated by relations of degree two and three. Furthermore, "type" is the only quadratic invariant on $\mathscr{V}_{\Xi}^{3}$, that is, if $\xi \sim \xi^{\prime}$ is a relation with $\xi$, $\xi^{\prime}$ $\in \mathscr{V}_{\Xi}^{3}$ then $\underline{\xi} \sim \underline{\xi}^{\prime}$ is essentially quadratic if and only if $t(\underline{\xi})=t\left(\underline{\xi^{\prime}}\right)$.

Theorem 4.1 follows easily from this, since generalized toric Segre cubics allow one to switch the type of a triple between $A$ and $B$ at any base vertex.

We now give an overview of the proof of Proposition 4.3. First of all we split relations into two types defined in $\$ 4.8$, "breakable" and "unbreakable." Breakable means that some degree one piece of the relation vanishes at some base edge. The first idea in the proof is that, given a relation, one can chop the tree into pieces such that the relation is unbreakable on each piece. One can then use a gluing argument to reduce to the unbreakable case. We then prove that an unbreakable relation is essentially quadratic. We do this by introducing a normal form for monomials and showing that any monomial can be changed into normal form by a series of quadratic relations. We use the notion of a "balanced" monomial, a tuple of matchings which assume roughly the same value on each edge of the tree. A key result (Proposition 4.4) is that any tuple can be balanced by quadratic relations.

\subsection{Balancing}

We say that a tuple of integers $\left(x_{i}\right)$ is balanced if $\left|x_{i}-x_{j}\right|$ is always 0 or 1 . We say that a tuple $\xi \in \mathscr{V}^{n}$ is balanced if for each base edge $e$ of $\Xi$ the tuple of integers $\left(\xi_{i}(e)\right)$ is balanced. 
Proposition 4.4. Given any tuple $\underline{\xi}$ in $\mathcal{V}^{n}$ there exists an essentially quadratic relation $\underline{\xi} \sim \underline{\xi}^{\prime}$ with $\underline{\xi}^{\prime}$ balanced.

Proof. It suffices to prove the proposition for $n=2$, as one can repeatedly balance pairs of integers to balance a set of integers. Thus suppose that $n=2$. First we show that the proposition holds for the third caterpillar tree. In the general case of the $r$ th caterpillar, we break $\Xi$ into its trinodes, balance the weightings on each of these separately and then "glue."

Third caterpillar: We indicate a reduced matching $\xi$ on the third caterpillar tree by a triple $(a, b, c)$ where $a$ is the weight of the first (left) stalk, $b$ is the weight of the second (vertical) stalk and $c$ is the weight of the third (right) stalk. The triple $(a, b, c)$ satisfies the triangle inequalities and has $b \leq 1$. If $b=0$ then $a=c$. If $b=1$ then one of $a$ or $c$ is non-zero, and $|a-c| \leq 1$. Suppose $\xi_{1}=\left(a_{1}, b_{1}, c_{1}\right)$ and $\xi_{2}=\left(a_{2}, b_{2}, c_{2}\right)$ are reduced matchings and $\left|a_{1}-a_{2}\right| \geq 2$ or $\left|c_{1}-c_{2}\right| \geq 2$. Without loss of generality we take $a_{1}+2 \leq a_{2}$. Since $c_{1} \leq a_{1}+1$ and $c_{2} \geq a_{2}-1$ we have $c_{1} \leq c_{2}$. We know that $c_{2} \geq 1$ since $a_{2} \geq 2$.

If $c_{1}<c_{2}$ then define $\xi_{1}^{\prime}=\left(a_{1}+1, b_{1}, c_{1}+1\right)$ and $\xi_{2}^{\prime}=\left(a_{2}-1, b_{2}, c_{2}-1\right)$. Then $\xi_{1}^{\prime}+\xi_{2}^{\prime}=\xi_{1}+\xi_{2}$ so $\left(\xi_{1}^{\prime}, \xi_{2}^{\prime}\right) \sim\left(\xi_{1}, \xi_{2}\right)$. The resulting pair $\left(\xi_{1}^{\prime}, \xi_{2}^{\prime}\right)$ is now closer to being balanced since $\left|\left(a_{1}+1\right)-\left(a_{2}-1\right)\right|=\left|a_{1}-a_{2}\right|-2$ and $\left|\left(c_{1}+1\right)-\left(c_{2}-1\right)\right| \leq\left|c_{1}-c_{2}\right|$.

If $c_{1}=c_{2}$ then $a_{2}=a_{1}+2$ and $c_{1}=c_{2}=a_{1}+1$. Define $\xi_{1}^{\prime}=\left(a_{1}+1, b_{1}, c_{1}\right)$ and $\xi_{2}^{\prime}=\left(a_{2}-1, b_{2}, c_{2}\right)$. Then $\xi_{1}^{\prime}$ and $\xi_{2}^{\prime}$ are reduced admissible matchings and $\left(\xi_{1}^{\prime}, \xi_{2}^{\prime}\right) \sim$ $\left(\xi_{1}, \xi_{2}\right)$. Again, we get strictly closer to a balanced pair with such an assignment.

A finite number of steps as above will relate the original pair $\left(\xi_{1}, \xi_{2}\right)$ to a balanced pair.

Breaking and gluing: Suppose now that we have a pair $\left(\xi_{1}, \xi_{2}\right)$ of reduced admissible matchings on the $r$ th caterpillar. Break the caterpillar up into an ordered tuple of $r-2$ caterpillar trees with three vertices by cutting each base edge in two. These are arranged from left to right and indexed as $2, \ldots, r-1$. The matchings $\xi_{i}$ on the original caterpillar define matchings on each copy of the third caterpillar. Let $e_{j}, f_{j}$ and $g_{j}$ denote the first, second and third stalk on the $j$ th trinode and define $\xi_{i, j}=\left(\xi_{i}\left(e_{j}\right), \xi_{i}\left(f_{j}\right), \xi_{i}\left(g_{j}\right)\right)$ for $i=1,2$ and $2 \leq j \leq r-1$. Now apply our previous result on the third caterpillar-each pair $\left(\xi_{1, j}, \xi_{2, j}\right)$ is equivalent to a balanced pair $\left(\xi_{1, j}^{\prime}, \xi_{2, j}^{\prime}\right)$.

Define $t_{2}=1$. Now, because the pairs are balanced, for $3 \leq j \leq r-1$ we have either $c_{1, j}^{\prime}=a_{1, j+1}^{\prime}$ and $c_{2, j}^{\prime}=a_{2, j+1}^{\prime}$, or $c_{1, j}^{\prime}=a_{2, j+1}^{\prime}$ and $c_{2, j}^{\prime}=a_{1, j+1}^{\prime}$. In the former case set $t_{j}=t_{j-1}$ while in the latter case set $t_{j}=3-t_{j-1}$. We will use the tuple $\left(t_{2}, \ldots, t_{r-1}\right)$ of 1's and 2's to glue these balanced pairs on individual trinodes to obtain a balanced pair on the $r$ th caterpillar.

We now define a pair $\left(\xi_{1}^{\prime}, \xi_{2}^{\prime}\right)$ of admissible weightings on the $r$ th caterpillar by a gluing procedure. Let the edges of the $r$ th caterpillar be labeled as $s_{1}, s_{2}, b_{2}, s_{3}, b_{3}, \ldots$, $b_{r-2}, s_{r-1}, s_{r}$, where $s_{j}$ is the $j$ th stalk and $b_{k}$ is the base edge between the $k$ th and $(k+1)$ st base vertices. To begin, we define $\xi_{1}^{\prime}$ (resp. $\left.\xi_{2}^{\prime}\right)$ on $s_{1}, s_{2}, b_{2}$ to agree with $\xi_{1,2}^{\prime}$ (resp. $\xi_{2,2}^{\prime}$ ). For $3 \leq j \leq r-3$ set

$$
\left(\xi_{1}^{\prime}\left(b_{j-1}\right), \xi_{1}^{\prime}\left(s_{j}\right), \xi_{1}^{\prime}\left(b_{j}\right)\right)=\xi_{t_{j}}^{\prime}, \quad\left(\xi_{2}^{\prime}\left(b_{j-1}\right), \xi_{2}^{\prime}\left(s_{j}\right), \xi_{2}^{\prime}\left(b_{j}\right)\right)=\xi_{2-t_{j}}^{\prime} .
$$


Finally define

$$
\left(\xi_{1}^{\prime}\left(b_{r-2}\right), \xi_{1}^{\prime}\left(s_{r-1}\right), \xi_{1}^{\prime}\left(s_{r}\right)\right)=\xi_{t_{r-1}}^{\prime}, \quad\left(\xi_{2}^{\prime}\left(b_{r-2}\right), \xi_{2}^{\prime}\left(s_{r-1}\right), \xi_{2}^{\prime}\left(s_{r}\right)\right)=\xi_{2-t_{r-1}}^{\prime} .
$$

The above assignments are well-defined, $\left(\xi_{1}^{\prime}, \xi_{2}^{\prime}\right)$ is balanced and $\left(\xi_{1}^{\prime}, \xi_{2}^{\prime}\right) \sim\left(\xi_{1}, \xi_{2}\right)$.

\subsection{Reduction of Proposition 4.3 to the unbreakable case (Proposition 4.5)}

We say that a matching $\xi$ on $\Xi$ is breakable if there exists a base edge $e$ with $\xi(e)=0$, and unbreakable otherwise. We say that a tuple of matchings $\underline{\xi} \in \mathscr{V}_{\Xi}^{n}$ is unbreakable if each $\xi_{i}$ is. In $\$ 4.9$ we will prove the following proposition:

Proposition 4.5. Let $\xi \sim \xi^{\prime}$ be a relation with $\underline{\xi}, \underline{\xi}^{\prime} \in \mathscr{V}_{\Xi}^{n}$ and $\underline{\xi}$ unbreakable. Then $\underline{\xi} \sim \underline{\xi}^{\prime}$ is essentially quadratic.

In this section we prove the following:

Proposition 4.6. Proposition 4.5 implies Proposition 4.3.

Proof. As remarked in $\S 4.4$, Proposition 4.3 is true for the third caterpillar tree. This will be the base case of an inductive argument.

Let $x \sim y$ be a relation of length $n$. Using quadratic relations, we may assume that both $x$ and $y$ are balanced. If each $x_{i}$ is unbreakable then each $y_{i}$ is as well (since $x$ and $y$ are balanced) and we are done. Assume then that there is a base edge $e$ for which $x_{i}$ is breakable at $e$ for some $i$.

Cut the edge $e$ in half to produce two new trees $\Xi^{\prime}$ and $\Xi^{\prime \prime}$. We regard $e$ as an edge of both of these trees. Both of these trees can be regarded as smaller caterpillar trees. Also, giving a weighting on $\Xi$ is equivalent to giving weightings on $\Xi^{\prime}$ and $\Xi^{\prime \prime}$ which agree at $e$.

Now, let $x^{\prime}$ and $x^{\prime \prime}$ be the restrictions of $x$ to $\Xi^{\prime}$ and $\Xi^{\prime \prime}$ (and similarly for $y$ ). The key point is that because $e$ is breakable and $x$ and $y$ are balanced, these restricted weightings are matchings, that is, they assign $e$ either 0 or 1 . In other words, we have $x^{\prime}, y^{\prime} \in \mathcal{V}_{\Xi^{\prime}}^{n}$ and $x^{\prime \prime}, y^{\prime \prime} \in \mathcal{V}_{\Xi^{\prime \prime}}^{n}$.

Proceeding by induction, we can assume that all relations are essentially cubic on $\Xi^{\prime}$ and $\Xi^{\prime \prime}$, or essentially quadratic if the types agree. We can then pick a sequence of cubic (resp. quadratic) relations between $x^{\prime}$ and $y^{\prime}$ and between $x^{\prime \prime}$ and $y^{\prime \prime}$ and concatenate them to form a sequence of cubic (resp. quadratic) relations between $x$ and $y$. By "concatenate," we mean that one should first order the tuples so that those taking value zero at the edge $e$ should be glued together (the order does not matter), and those taking value one at $e$ should be glued together (the order does not matter). In the final step-that is, after $x^{\prime}$ has been replaced with $y^{\prime}$ (up to permutation) and $x^{\prime \prime}$ has been replaced with $y^{\prime \prime}$ (up to permutation) - one can finally permute the $y^{\prime \prime}$ matchings taking value zero at $e$, and permute those taking value one at $e$, and lastly permute the concatenated matchings, so that the result is equal to $y$. (Recall that permutations are essentially quadratic since they are generated by 2 -cycles.) 


\subsection{Proof of Proposition 4.5}

There are four possibilities for an unbreakable matching at the trinodes on the left end:

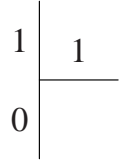

$A$

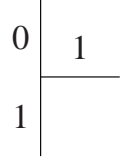

$B$

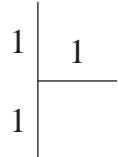

C

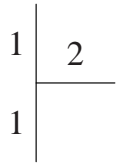

$D$

The right end is similar (flip each over). We order these: $A \leq B \leq C \leq D$.

We say that an unbreakable tuple of matchings $\underline{\xi} \in \mathscr{V}_{\Xi}^{n}$ is in normal form if $\underline{\xi}$ is balanced and the following conditions hold:

- The restriction of the sequence $\left(\xi_{i}\right)$ to the leftmost trinode is non-decreasing, under the order described above; similarly for the rightmost trinode.

- For each base edge $e$, the sequence $\left(\xi_{i}(e)\right)$ is non-decreasing.

- If $e$ and $e^{\prime}$ are consecutive base edges and $\xi_{i}(e)=\xi_{j}(e)$ and $\xi_{i}\left(e^{\prime}\right)=\xi_{j}\left(e^{\prime}\right)$ for some $i \leq j$ then $\xi_{i}(s) \leq \xi_{j}(s)$, where $s$ is the stalk between $e$ and $e^{\prime}$.

- If $e$ and $e^{\prime}$ are consecutive base edges and $\xi_{i}(e)=\xi_{i}\left(e^{\prime}\right)$ and $\xi_{j}(e)=\xi_{j}\left(e^{\prime}\right)$ for some $i \leq j$ then $\xi_{i}(s) \leq \xi_{j}(s)$, where $s$ is the stalk between $e$ and $e^{\prime}$.

Proposition 4.5 now follows from the following proposition:

Proposition 4.7. Every unbreakable element of $\mathscr{V}_{\Xi}^{n}$ is related to a unique normal form, and this relation is essentially quadratic.

The following is the key lemma:

Lemma 4.8. Let $\left(\xi, \xi^{\prime}\right) \in \mathscr{V}_{\Xi}^{2}$ be unbreakable and balanced. Then there exists $\left(\eta, \eta^{\prime}\right)$ $\in \mathscr{V}_{\Xi}^{2}$ balanced and unbreakable such that $\xi+\xi^{\prime}=\eta+\eta^{\prime}$ and for each internal edge $e$ we have

$$
\eta(e)=\min \left(\xi(e), \xi^{\prime}(e)\right), \quad \eta^{\prime}(e)=\max \left(\xi(e), \xi^{\prime}(e)\right) .
$$

Proof. The idea is the same as in the proof of Proposition 4.4: break apart at a base edge (or at an end), permute, and glue back together to achieve the desired order.

We now prove Proposition 4.7.

Proof of Proposition 4.7. Let $\xi \in \mathscr{V}_{\Xi}^{n}$ be a given unbreakable tuple. We may assume that $\xi$ is balanced. By repeatedly using Lemma 4.8 we find that $\xi$ is quadratically related to an unbreakable element $\underline{\xi}^{\prime} \in \mathscr{V}_{\Xi}^{n}$ which has the property that $\bar{\xi}_{i}^{\prime}(e) \leq \xi_{j}^{\prime}(e)$ for $i \leq j$ and all internal edges $e$.

For uniqueness, it suffices to show uniqueness for third caterpillars and for the ends. Consider the third caterpillar at a base vertex. Suppose the sum of the $n$ weightings $\left(a_{i}, b_{i}, c_{i}\right), 1 \leq i \leq n$, on the third caterpillar is equal to $(a, b, c)$ (left, stalk, right). Suppose these weightings are increasing in the order we have defined. This means the $a_{i}$ 's are increasing, starting as the floor of $a / n$ and ending as the ceiling of $a / n$. This determines the value of each $a_{i}$. Similarly the value of each $c_{i}$ is determined (beginning 
with floor of $c / n$, ending with ceiling of $c / n$ ). Wherever $a_{i} \neq c_{i}$ we must have $b_{i}=1$, so these $b_{i}$ 's are determined. However if $a_{i}=c_{i}$, then $b_{i}$ could be either 0 or 1 . The set $\left\{i \mid a_{i}=c_{i}\right\}$ consists of at most two intervals $I, J$. There is some integer $n$ such that $I=\left\{i \mid a_{i}=c_{i}=n\right\}$ and $J=\left\{j \mid a_{j}=c_{j}=n+1\right\}$. Within the interval $I$, the $b_{i}$ 's must form a non-decreasing sequence. Similarly in the interval $J$, the $b_{i}$ 's form a non-decreasing sequence. We also know the value of $\sum_{i \in I \cup J} b_{i}$, and that we cannot have any pair $i \in I, j \in J$ such that $b_{i}=1$ and $b_{j}=0$. These conditions together determine the values of all the $b_{i}$ 's. The argument for the ends is similar.

\section{The ideal is generated by quadratics and generalized Segre cubics}

In $\S 5$, we lift the generalized Segre toric cubics to the ring $R_{L}$ and prove the following:

Theorem 5.1. For any even set $L$ the ideal $I_{L}$ is generated over $\mathbb{Z}$ by quadratics and the small generalized Segre cubic relations.

We will introduce the generalized Segre cubics, and the small generalized Segre cubics, shortly. Theorem 5.1 will follow easily from our toric results once we make these definitions. In one of our ad hoc arguments in the 10-point case we will need a slightly refined version of Theorem 5.1 given in Remark 5.3. The proof of this theorem is the only place we use the toric results.

\subsection{Brief additional comments on colored graphs}

We will use the language of colored graphs, introduced in $\$ 1$. We consider both directed and undirected colored graphs. If $\Gamma$ is a directed multi-matching on $L$ whose edges have been colored with colors from the set $C$ then $X_{\Gamma}$ is defined as an element of $V_{L}^{\otimes C}$ as in $\S 1$. If $L$ is oriented and $\Gamma$ is a regular undirected multi-matching one can also make sense of $Y_{\Gamma}$ as an element of $V_{L}^{\otimes C}$. Clearly the $X_{\Gamma}$ (or $Y_{\Gamma}$ ) span $V_{L}^{\otimes C}$. Furthermore, the $X_{\Gamma}$ satisfy the sign and "colored" Plücker relations, and these generate all the linear relations among them. (The colored Plücker relation is just the usual Plücker relation on a pair of edges, with the restriction that these two edges be of the same color.) We have thus given a description of $V_{L}^{\otimes C}$ in terms of colored graphs. There is a similar description for $\operatorname{Sym}^{k}\left(V_{L}\right)$. The only difference is that in $\operatorname{Sym}^{k}\left(V_{L}\right)$ the particular color of an edge is not relevant. What matters is whether two edges have the same color-two colored graphs represent the same element of $\operatorname{Sym}^{k}\left(V_{L}\right)$ if one is obtained from the other by permuting the colors.

\subsection{Generalized Segre cubic data}

Let $L$ be an even set. By a generalized Segre datum we mean a pair $\Sigma=(\Gamma, \mathscr{U})$ where $\Gamma$ is an undirected graph on $L$ whose edges have been colored one of red/dashed, green/solid or blue/dotted and $\mathscr{U}=\left\{U_{R}, U_{G}, U_{B}\right\}$ is a partition of $L$ into three even subsets (called parts), such that 
- Every vertex of $\Gamma$ has valence one for each of the three colors.

- Between any two parts there are either two edges or no edges. If there are two edges then these edges have the color of the "opposite" part. For instance, any edge from $U_{G}$ to $U_{B}$ must be dashed.

See Figure 5 for a schematic presentation. We call the edges between the parts special. We call $\Sigma$ small if $U_{R}, U_{G}$ or $U_{B}$ has cardinality two.

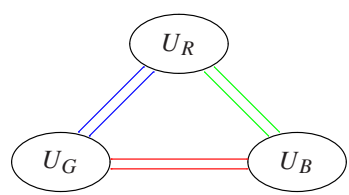

Fig. 5. Schematic presentation of a generalized Segre datum.

Let $\Sigma$ be a generalized Segre datum. Suppose $e$ and $e^{\prime}$ are edges of $\Gamma$ of the same color and have a vertex in a common part. Given such a pair, let $Y_{\Gamma}+Y_{\Gamma^{\prime}}+Y_{\Gamma^{\prime \prime}}=0$ be the colored Plücker relation on $e$ and $e^{\prime}$. Then it is easily verified that $\Sigma^{\prime}=\left(\Gamma^{\prime}, \mathscr{U}\right)$ and $\Sigma^{\prime \prime}=\left(\Gamma^{\prime \prime}, \mathscr{U}\right)$ are both generalized Segre data. Define the space of generalized Segre data to be the $\mathbb{Z}$-module spanned by generalized Segre data modulo relations of the form $\Sigma+\Sigma^{\prime}+\Sigma^{\prime \prime}=0$.

\subsection{Generalized Segre cubic relations}

Let $\Sigma=(\Gamma, \mathscr{U})$ be a generalized Segre datum. Let $\widetilde{\Gamma}$ be a directed colored graph with underlying undirected graph $\Gamma$. We let $\epsilon(\widetilde{\Gamma})$ be the product of the $\epsilon\left(\widetilde{\Gamma}_{i}\right)$, where $\widetilde{\Gamma}_{i}$ is the directed matching of color $i$ in $\widetilde{\Gamma}$. (Here $\epsilon$ is a chosen orientation on $L$, see $\S 2.2$.) Form a new directed colored graph $\widetilde{\Gamma}^{\prime}$ as follows. The graph $\widetilde{\Gamma}^{\prime}$ will be a recoloring of $\widetilde{\Gamma}$, so we just specify a new color for each edge. We use the colors purple/light and black/dark. The dashed (resp. dotted, solid) edges of $\widetilde{\Gamma}$ in $U_{R}$ (resp. $U_{B}, U_{G}$ ) are dark in $\widetilde{\Gamma}^{\prime}$ and all other edges of $\widetilde{\Gamma}$ are light in $\widetilde{\Gamma}^{\prime}$. It is clear that every vertex has dark valence one and thus light valence two.

Define $Y_{\Sigma} \in \operatorname{Sym}^{3}\left(V_{L}\right)$ and $Y_{\Sigma}^{\prime} \in R_{L}^{(1)} \otimes R_{L}^{(2)}$ by

$$
Y_{\Sigma}=Y_{\Gamma}=\epsilon(\widetilde{\Gamma}) X_{\widetilde{\Gamma}}, \quad Y_{\Sigma}^{\prime}=\epsilon(\widetilde{\Gamma})\left(X_{\widetilde{\Gamma}_{\text {dark }}^{\prime}} \otimes X_{\widetilde{\Gamma}_{\text {light }}^{\prime}}\right) .
$$

These only depend on $\Sigma$ and not the choice of $\widetilde{\Gamma}$. There is a well-defined map $R_{L}^{(1)} \otimes$ $R_{L}^{(2)} \rightarrow \operatorname{Sym}^{3}\left(V_{L}\right) / Q_{L}^{(3)}$ given by writing the element of $R_{L}^{(2)}$ in terms of degree one elements and then formally multiplying to get an element of $\operatorname{Sym}^{3}\left(V_{L}\right)$. (This is only defined modulo quadratic relations $Q_{L}$, because of the choice of how to write the element of $R_{L}^{(2)}$ in terms of degree one elements.) We may therefore regard both $Y_{\Sigma}$ and $Y_{\Sigma}^{\prime}$ as elements of $\operatorname{Sym}^{3}\left(R_{L}^{(1)}\right) / Q_{L}$. Define

$$
\operatorname{Rel}(\Sigma)=Y_{\Sigma}-Y_{\Sigma}^{\prime}
$$


regarded as an element of $\operatorname{Sym}^{3}\left(V_{L}\right) / Q_{L}^{(3)}$. As the two terms in $\operatorname{Rel}(\Sigma)$ are recolorings of the same graph, $\operatorname{Rel}(\Sigma)$ is a relation, that is, it maps to zero in $R_{L}$. We call such relations generalized Segre cubic relations. We use the same name for lift of some $\operatorname{Rel}(\Sigma)$ to $I_{L}^{(3)} \subset \operatorname{Sym}^{3}\left(V_{L}\right)$. A generalized Segre cubic relation is shown in Figure 6. Note that for $\sigma \in \mathfrak{S}_{L}$ we have $\sigma \operatorname{Rel}(\Sigma)=\operatorname{sgn}(\sigma) \operatorname{Rel}(\sigma \Sigma)$.

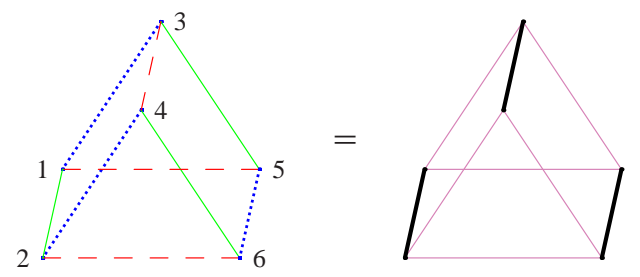

Fig. 6. A generalized Segre relation on six points. Here $U_{G}=\{1,2\}, U_{R}=\{3,4\}$ and $U_{B}=$ $\{5,6\}$. This is equal to the image of the usual Segre cubic relation in $I_{L} / Q_{L}$.

One easily verifies that Rel gives a homomorphism

Rel : $\{$ the space of generalized Segre cubic data $\} \rightarrow I_{L}^{(3)} / Q_{L}^{(3)}$.

This allows us to interpret relations as graphs with extra structure, which has two advantages. First, it gives a source of linear relations between (also thought of as operations we can perform on) generalized Segre relations: Plücker relations on the generalized Segre data which respect the extra structure. Second, one can read off certain properties of the generalized Segre relation from the original graph. For instance, if $\Sigma$ is a generalized Segre cubic datum for which $\Gamma$ is disconnected, then the associated generalized Segre relation arises from a relation on fewer points (see Proposition 7.3 for a precise statement).

\subsection{Degenerate Segre cubic relations}

We call a generalized Segre datum $\Sigma$ degenerate if:

(1) one of the pairs of special edges is missing; or

(2) in one of the parts the two pairs of special edges do not connect.

By (2) we mean that one part, say $U_{G}$, can be partitioned into two pieces $A$ and $B$ such that no edges go between $A$ and $B$, the dotted special edges go into $A$ and the dashed special edges go into $B$. A generalized Segre relation is called degenerate if it comes from a degenerate generalized Segre datum.

Proposition 5.2. A degenerate generalized Segre relation lies in $Q_{L}$.

Proof. First consider a degenerate datum satisfying (1). Say that the dashed special edges are missing. We show how to move from $Y_{\Sigma}$ to $Y_{\Sigma}^{\prime}$ using quadratic relations. First switch the dashed edges and the solid edges of $\Gamma$ which occur in $U_{G}$ (this relation lies in $Q_{L}$ ). Then switch the dashed edges and dotted edges of $\Gamma$ in $U_{B}$. The resulting graph now looks like $\Gamma^{\prime}$ if we make the dashed edges dark and the other edges light. 
Now say that $\Sigma$ is a degenerate datum satisfying (2). Say that in $U_{G}$ the dashed and dotted special edges do not connect and let $U_{G}=A \amalg B$ be a decomposition as described above. Let $\Gamma^{\prime}$ be the graph obtained from $\Gamma$ by switching the dashed and solid colors in $A$, let $U_{G}^{\prime}=B$, let $U_{R}^{\prime}=U_{R} \cup A$ and let $U_{B}^{\prime}=U_{B}$. Let $\Sigma^{\prime}$ be the generalized Segre datum $\left(\Gamma^{\prime},\left\{U_{R}^{\prime}, U_{G}^{\prime}, U_{B}^{\prime}\right\}\right)$. Then $\Sigma^{\prime}$ is degenerate of type (1) and so $\operatorname{Rel}\left(\Sigma^{\prime}\right) \in Q_{L}$. Consider the difference

$$
\operatorname{Rel}(\Sigma)-\operatorname{Rel}\left(\Sigma^{\prime}\right)=\left(Y_{\Sigma}-Y_{\Sigma^{\prime}}\right)+\left(Y_{\Sigma}^{\prime}-Y_{\Sigma^{\prime}}^{\prime}\right)
$$

Now $Y_{\Sigma}^{\prime}=Y_{\Sigma^{\prime}}^{\prime}$. Also, $Y_{\Sigma}-Y_{\Sigma^{\prime}} \in Q_{L}$ (the dotted subgraphs of $Y_{\Sigma}$ and $Y_{\Sigma^{\prime}}$ are identical, and the remainder of the graph gives a quadratic relation). Thus $\operatorname{Rel}(\Sigma) \in Q_{L}$.

\subsection{Proof of Theorem 5.1}

It follows from Theorem 4.1 that $I_{L}$ is generated by quadratics and lifts of the generalized toric Segre cubics. Thus to prove Theorem 5.1, it suffices to show that the generalized Segre cubics are lifts of the generalized toric Segre cubics, which is what we now do.

Let $L$ be an even set. Pick a Y-tree $\Xi$ with leaf set $L$ and let $\Xi^{-}$be the associated reduced (caterpillar) tree. Consider a generalized toric Segre cubic relation on $\Xi^{-}$:

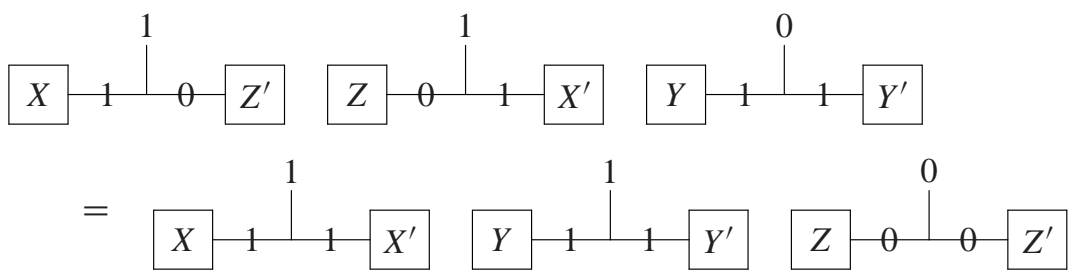

Implicit in this diagram is a decomposition of $\Xi^{-}$into three pieces:
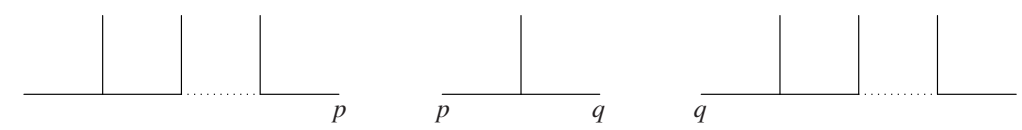

The labels indicate how these three pieces are glued together (after gluing, $p$ and $q$ disappear). Corresponding to this decomposition is a decomposition of $L$ into three pieces, the left piece $P$, the right piece $P^{\prime}$ and the center piece, which has two elements $x$ and $y$ (the two vertices of the Y-tree $\Xi$ which connect to the stalk of the trinode in the center of the above diagram). We regard $X, Y$ and $Z$ as reduced degree one weightings on the left caterpillar tree, where $X$ and $Y$ take value 1 on $p$ while $Z$ takes value 0 there. Similarly, we regard $X^{\prime}, Y^{\prime}$ and $Z^{\prime}$ as reduced weightings on the right caterpillar tree, where $X^{\prime}$ and $Y^{\prime}$ take value 1 on $q$ while $Z^{\prime}$ takes value 0 there.

Now, we can regard $X, Y$ and $Z$ as non-reduced degree one weightings on the Y-tree with vertex set $P \cup\left\{p^{+}, p^{-}\right\}$. Similarly, we can regard $X^{\prime}, Y^{\prime}$ and $Z^{\prime}$ as non-reduced degree one weightings on the Y-tree with vertex set $P^{\prime} \cup\left\{q^{+}, q^{-}\right\}$. We lift these six weightings to matchings, which we denote by $\widetilde{X}$, etc. In the graph $\widetilde{X}$ there are two elements of $P$, say $a$ and $b$, which connect to $p^{+}$and $p^{-}$. We let $\widetilde{X}_{0}$ be the restriction of $\widetilde{X}$ to $P \backslash\{a, b\}$. We let $c$ and $d$ be the two vertices in $\widetilde{Y}$ connecting to $p^{+}$and $p^{-}$and let $\widetilde{Y}_{0}$ 
be the restriction of $\widetilde{Y}$ to $P \backslash\{c, d\}$. In $\widetilde{Z}$ the vertices $p^{+}$and $p^{-}$are connected to each other. We similarly define primed versions.

We now define a small generalized Segre cubic datum $\Sigma$. The partition is given by $U_{B}=P^{\prime}, U_{R}=\{x, y\}$ and $U_{G}=P$. The graph $\Gamma$ is given as follows:

- The dotted graph is the union of $\widetilde{X}_{0}, \widetilde{Z}^{\prime}$ and the edges $\overline{a x}$ and $\overline{b y}$.

- The solid graph is the union of $\widetilde{Z}, \widetilde{X}_{0}^{\prime}$ and the edges $\overline{a^{\prime} x}$ and $\overline{b^{\prime} y}$.

- The dashed graph is the union of $\widetilde{Y}_{0}, \widetilde{Y}_{0}^{\prime}$ and the edges $\overline{x y}, \overline{c c^{\prime}}$ and $\overline{d d^{\prime}}$.

These three matchings are lifts for the three reduced weightings appearing on the left side of (5.1). It is clear that this is a valid small generalized Segre cubic datum.

We must now show that the generalized Segre cubic relation $\operatorname{Rel}(\Sigma)$ associated to the generalized Segre cubic datum defined above lifts the relation (5.1). Now, the relation associated to the datum is

$$
\begin{aligned}
\left(\tilde{X}_{0} \cdot \overline{a x} \cdot \overline{b y} \cdot \widetilde{Z}^{\prime}\right) & \left(\widetilde{Z} \cdot \overline{a^{\prime} x} \cdot \overline{b^{\prime} y} \cdot \tilde{X}_{0}^{\prime}\right)\left(\tilde{Y} \cdot \overline{c c^{\prime}} \cdot \overline{d d^{\prime}} \cdot \overline{x y} \cdot \widetilde{Y}^{\prime}\right) \\
= & \left(\widetilde{X}_{0} \cdot \widetilde{Y}_{0} \cdot \overline{a x} \cdot \overline{a^{\prime} x} \cdot \overline{b y} \cdot \overline{b^{\prime} y} \cdot \overline{c c^{\prime}} \cdot \overline{d d^{\prime}} \cdot \tilde{X}_{0}^{\prime} \cdot \widetilde{Y}_{0}^{\prime}\right)\left(\widetilde{Z} \cdot \overline{x y} \cdot \widetilde{Z}^{\prime}\right) .
\end{aligned}
$$

Here the parenthesized expressions should each be interpreted as single graphs-for example, the first parenthetical is the concatenation of $\widetilde{X}_{0}, \overline{a x}, \overline{b y}$ and $\widetilde{Z}^{\prime}$. The quadratic term $\Delta$ on the right side would be the light subgraph in the graphical notation. Now, the above relation belongs to $I_{L}^{(3)} / Q_{L}^{(3)}$. To get an element of $I_{L}^{(3)}$, we must rewrite $\Delta$ in terms of degree one elements. Because the toric ring $\operatorname{gr}_{\Xi} R_{L}$ is generated in degree one, we can write

$$
\Delta=\sum_{i=1}^{n} \Phi_{i} \Phi_{i}^{\prime}
$$

where each $\Phi_{i}$ and $\Phi_{i}^{\prime}$ has degree one, $\Phi_{1} \Phi_{1}^{\prime}$ has toric weight equal to that of $\Delta$, and $\Phi_{i} \Phi_{i}^{\prime}$ has toric weight strictly less than that of $\Delta$ for $i>1$. We may take $\Phi_{1}=\widetilde{X}_{0} \cdot \overline{a x}$. $\overline{a^{\prime} y} \cdot \overline{b b^{\prime}} \cdot \widetilde{X}_{0}^{\prime}$ and $\Phi_{1}^{\prime}=\widetilde{Y}_{0} \cdot \overline{c x} \cdot \overline{c^{\prime} y} \cdot \overline{d d^{\prime}} \cdot \widetilde{Y}_{0}^{\prime}$, since the product of these two graphs is equal to $\Delta$ in the toric ring.

It now follows that $\operatorname{Rel}(\Sigma)$ is represented by the relation

$$
\left(\widetilde{X}_{0} \cdot \overline{a x} \cdot \overline{b y} \cdot \widetilde{Z}^{\prime}\right)\left(\widetilde{Z} \cdot \overline{a^{\prime} x} \cdot \overline{b^{\prime} y} \cdot \widetilde{X}_{0}^{\prime}\right)\left(\widetilde{Y} \cdot \overline{c c^{\prime}} \cdot \overline{d d^{\prime}} \cdot \overline{x y} \cdot \widetilde{Y}^{\prime}\right)=\left(\sum_{i=1}^{n} \Phi_{i} \Phi_{i}^{\prime}\right)\left(\widetilde{Z} \cdot \overline{x y} \cdot \widetilde{Z}^{\prime}\right) .
$$

The leading term (in terms of the grading) of this relation is

$$
\left(\tilde{X}_{0} \cdot \overline{a x} \cdot \overline{b y} \cdot \widetilde{Z}^{\prime}\right)\left(\widetilde{Z} \cdot \overline{a^{\prime} x} \cdot \overline{b^{\prime} y} \cdot \tilde{X}_{0}^{\prime}\right)\left(\tilde{Y} \cdot \overline{c c^{\prime}} \cdot \overline{d d^{\prime}} \cdot \overline{x y} \cdot \tilde{Y}^{\prime}\right)=\left(\Phi_{1} \Phi_{1}^{\prime}\right)\left(\widetilde{Z} \cdot \overline{x y} \cdot \widetilde{Z}^{\prime}\right) \text {. }
$$

The two sides are the same as the two sides of (5.1). This shows that the leading term of our generalized Segre relation $\operatorname{Rel}(\Sigma)$ is equal to the generalized toric Segre relation we started with, which proves Theorem 5.1.

Remark 5.3. It follows from the proof that, if we totally order $L$, then $I_{L}$ is generated by quadratic relations and those small generalized Segre cubic relations coming from data for which $U_{G}<U_{R}<U_{B}$ and $\left|U_{R}\right|=2$. We will use this stronger form of Theorem 5.1 in the proof of Proposition 7.9. 


\section{The structure of $V_{L}$ and its tensor powers}

In $\S 6$, we study the partition filtration on $\otimes^{n} V_{L}$, $\operatorname{Sym}^{n}\left(V_{L}\right)$ and $\bigwedge^{n} V_{L}$ for $n \leq 3$ and the $\mathfrak{S}_{L}$-action on the associated graded pieces. This provides us with essential structural properties of the Plücker relation; for example: the elements $\left(Y_{\Gamma}\right)^{2}$ with $\Gamma$ a matching span $\operatorname{Sym}^{2}\left(V_{L}\right)$ (see the discussion following Proposition 6.1) and useful generalizations.

\subsection{The partition filtration}

Let $L$ be an even set. By a partition of $L$ we mean a collection of non-empty disjoint subsets of $L$ whose union is $L$. We say that a partition is into even parts if each of the subsets is even. We similarly speak of partitions of $|L|$ into even parts. Given a partition $\mathscr{U}$ of $L$ we denote by $|\mathscr{U}|$ the corresponding partition of $|L|$. We partially order the set of partitions of $L$ and $|L|$ by refinement. For example $2+2+2+2$ is smaller than $4+2+2$, but $4+4$ and $6+2$ are not comparable.

Given a regular colored graph $\Gamma$ on $L$ we obtain a partition $\mathscr{U}_{\Gamma}$ of $L$ by taking the vertex sets of the connected components of $\Gamma$. The partition $\mathscr{U}_{\Gamma}$ necessarily has even parts. We use this to define a filtration, indexed by the partitions of $|L|$ into even parts, on any $\mathbb{Z}$-module $U$ which is spanned by graphs. We denote this filtration, which we call the partition filtration, by $F_{p} U$. (Warning: this filtration is indexed by a partially ordered set, not a totally ordered set.) For instance, if $p$ is a partition of $|L|$ then $F_{p} \operatorname{Sym}^{k}\left(V_{L}\right)$ is the span of the $X_{\Gamma}$ (or $Y_{\Gamma}$ ) for which $\left|\mathscr{U}_{\Gamma}\right| \leq p$. We denote the associated graded subquotient by $\operatorname{gr}_{p} U$. To be precise, $\operatorname{gr}_{p} U$ is the quotient of $F_{p} U$ by the span of the $F_{p^{\prime}} U$ with $p^{\prime}<p$. The partition filtration is preserved by the action of $\mathfrak{S}_{L}$, so this group naturally acts on $\operatorname{gr}_{p} U$.

\subsection{Degree one spaces}

The $n=1$ case is easy: the only non-zero piece of the partition filtration on $V_{L}$ occurs for $p=2+\cdots+2$ and then $\operatorname{gr}_{p}=V_{L}$ is the irreducible representation of $\mathfrak{S}_{L}$ corresponding to the partition $n / 2+n / 2(\S 1.5)$.

\subsection{Degree two spaces}

We now study the partition filtration on $V_{L}^{\otimes 2}, \operatorname{Sym}^{2}\left(V_{L}\right)$ and $\bigwedge^{2} V_{L}$.

Proposition 6.1. We have, over $\mathbb{Z}[1 / 2]$ :

$$
\operatorname{gr}_{p}\left(V_{L}^{\otimes 2}\right)= \begin{cases}\operatorname{Sym}^{2}\left(V_{L}\right) & \text { if } p=2+\cdots+2, \\ \wedge^{2} V_{L} & \text { if } p=4+2+\cdots+2, \\ 0 & \text { otherwise. }\end{cases}
$$

The space $V_{L}^{\otimes 2}$ is spanned by regular 2-colored graphs. Such graphs are disjoint unions of cycles of even size. Proposition 6.1 says that $V_{L}^{\otimes 2}$ is spanned by graphs which are unions of 2-cycles and at most one 4-cycle. Furthermore, $\operatorname{Sym}^{2}\left(V_{L}\right)$ is spanned by graphs which are unions of 2-cycles: the elements $\left(Y_{\Gamma}\right)^{2}$ with $\Gamma$ a matching span $\operatorname{Sym}^{2}\left(V_{L}\right)$. 
We begin our proof of Proposition 6.1 with the following result:

Lemma 6.2. The space $V_{L}^{\otimes 2}$ is spanned over $\mathbb{Z}[1 / 2]$ by graphs which are unions of 2cycles and 4-cycles.

Proof. It suffices, by induction, to show that every regular 2-colored graph on at least six vertices can be written as a sum of graphs which are not connected. In other words, letting $U_{L}$ be the subspace of $V_{L}^{\otimes 2}$ spanned by disconnected graphs, it suffices to show that $V_{L}^{\otimes 2} / U_{L}$ is zero. Thus let $\Gamma$ be a graph on $L$, which we can assume to be connected (otherwise it already belongs to $U_{L}$ ). We must show $Y_{\Gamma}=0$ in $V_{L}^{\otimes 2} / U_{L}$.

Pick four consecutive vertices $a, b, c$ and $d$ of $\Gamma$. The Plücker relation on the edges $\overline{a b}$ and $\overline{c d}$ is

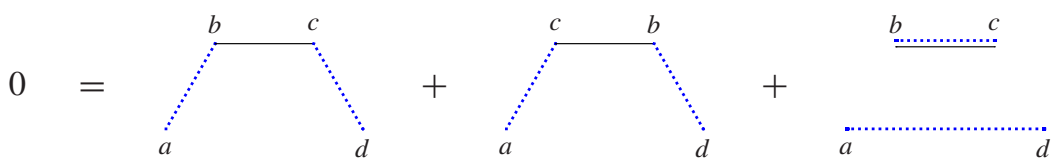

The rightmost term belongs to $U_{L}$. We therefore have $Y_{\Gamma}=-Y_{\Gamma^{\prime}}$ in $V_{L}^{\otimes 2} / U_{L}$ where $\Gamma^{\prime}$ is obtained by transposing two consecutive vertices in $\Gamma$. In words, we may transpose consecutive vertices at the cost of a sign, modulo $U_{L}$.

Now consider six consecutive vertices $a, b, c, d, e$ and $f$ in $\Gamma$. Applying the Plücker relation on the edges $\overline{a b}$ and $\overline{e f}$ we find

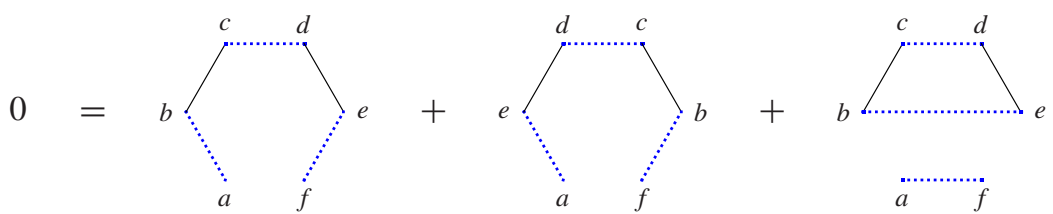

Again, the rightmost term belongs to $U_{L}$ and thus can be discarded. This shows that we may pick four consecutive vertices and reverse their order at the cost of picking up a sign. However, we may now move the affected vertices back to the original position using six transpositions, not introducing a new sign. We thus find that $Y_{\Gamma}=-Y_{\Gamma}$ in $V_{L}^{\otimes 2} / U_{L}$, which shows that $2 Y_{\Gamma}$ belongs to $U_{L}$.

In the proof of Lemma 6.2 we only ever use six consecutive vertices. By keeping track of the graphs we discarded during the course of the proof we obtain an identity, shown in Figure 7, that we will use on a few later occasions.

Lemma 6.2 states that $V_{L}^{\otimes 2}$ is spanned by graphs with only 2- and 4-cycles. To prove Proposition 6.1, we must therefore show that two 4-cycles can be converted into two 2cycles and one 4-cycle. This is a question about graphs on eight points; in fact, it suffices to show $\operatorname{gr}_{4,4}\left(V_{L}^{\otimes 2}\right)=0$ when $L$ has cardinality eight. We prove this after the following lemma.

Lemma 6.3. Let $L$ be an even set and let $\Gamma$ be a regular 2-colored graph on L. Put $p=\left|\mathscr{U}_{\Gamma}\right|$. Assume that $\Gamma$ has a 4 -cycle and let $\Gamma^{\prime}$ be the graph obtained by switching the colors of $\Gamma$ on this 4-cycle. Then $Y_{\Gamma}=-Y_{\Gamma^{\prime}}$ holds in $\operatorname{gr}_{p}\left(V_{L}^{\otimes 2}\right)$. 

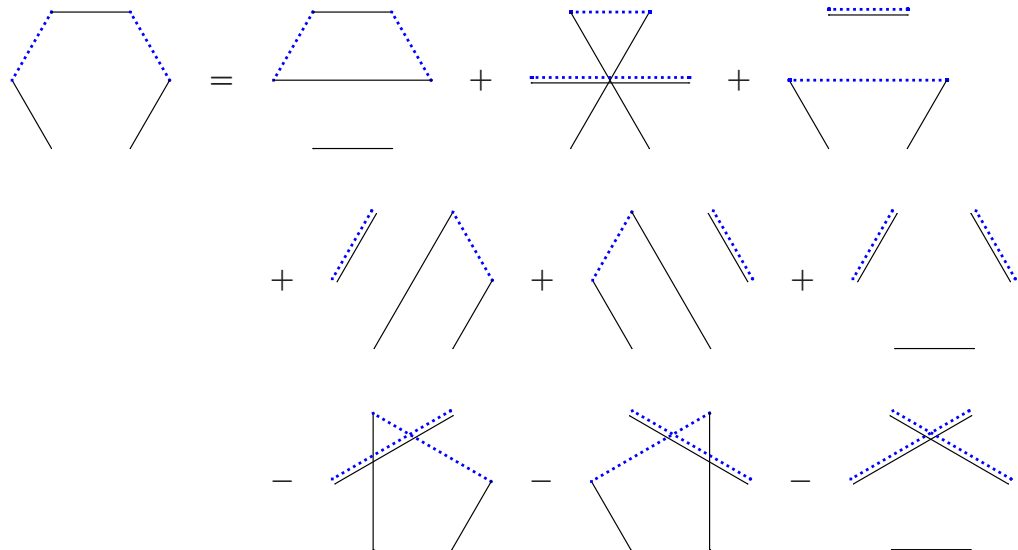

Fig. 7. A graphical identity on six points. More accurately, this should be regarded as a family of identities between various tensors $Y_{\Gamma} \otimes Y_{\Delta}$ in $V_{L}^{\otimes 2}$ for any $L$; we have only drawn the edges on each side which are different.

Proof. Let $\Gamma$ be given and say that the vertices in the 4-cycle are labeled $\{1,2,3,4\}$. By squaring the identity

$$
(-1)
$$

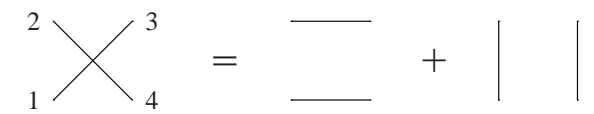

we obtain

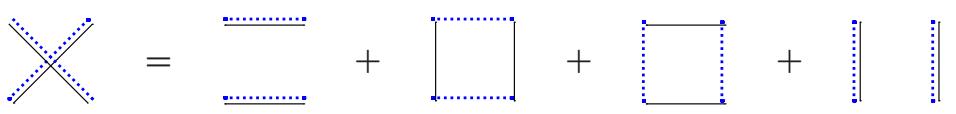

The middle two terms on the right side of this identity are $\Gamma$ and $\Gamma^{\prime}$. The other terms have a more refined partition and so map to 0 in $\operatorname{gr}_{p}\left(V_{L}^{\otimes 2}\right)$. This proves the lemma.

Lemma 6.4. Let $L$ be a set of cardinality eight. Then $\operatorname{gr}_{4,4}\left(V_{L}^{\otimes 2}\right)=0$ over $\mathbb{Z}[1 / 2]$.

Proof. In this proof we write $F_{p}$ for $F_{p}\left(V_{L}^{\otimes 2}\right)$, and similarly for $\operatorname{gr}_{p}$. Let $\tau$ be the involution on $V_{L}^{\otimes 2}$ which transposes factors. In terms of colored graphs, $\tau$ switches the colors. We will show that $\tau$ acts by +1 and by -1 on gr $_{4,4}$, which will establish the lemma.

We first show that $\tau$ acts by -1 on $\operatorname{gr}_{4,4}$. By Lemma 6.2 we have $F_{4,4}=V_{L}^{\otimes 2}$ and $F_{6,2}=F_{4,2,2}$. Thus $\operatorname{gr}_{4,4}$ can be described as the space of all degree two multi-matchings on $L$ modulo the disconnected ones. It therefore suffices to show that $\tau$ acts by -1 on a connected graph. Consider such a graph $\Gamma$, say the following one:

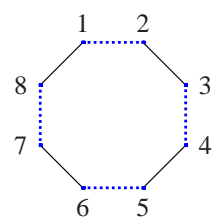


By the same reasoning as in the proof of Lemma 6.2 we may switch consecutive vertices at the cost of a sign, when working modulo disconnected graphs. Thus we may pick vertex 1 and move it counterclockwise around the cycle to the position of vertex 8 . This uses seven transpositions and so introduces a sign. The resulting cycle is the same as the original but with the colors switched. We thus have $\tau \Gamma=-\Gamma$ modulo disconnected graphs, which shows that $\tau$ acts by -1 on $\operatorname{gr}_{4,4}$.

We now show that $\tau$ acts by +1 on $\mathrm{gr}_{4,4}$. To do this, it suffices to show that $\tau$ fixes the graph

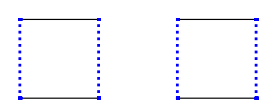

modulo $\operatorname{gr}_{4,2,2}$. This follows immediately from Lemma 6.3: switching the color in one square introduces a sign modulo $\mathrm{gr}_{4,2,2}$, so switching the color in both squares introduces no sign.

We now complete the proof of Proposition 6.1.

Proof of Proposition 6.1. Combining Lemma 6.2 with Lemma 6.4 shows that $\operatorname{gr}_{p}\left(V_{L}^{\otimes 2}\right)$ $=0$ unless $p=2+\cdots+2$ or $p=4+2+\cdots+2$. We thus have a filtration

$$
0 \subset F_{2+\cdots+2}\left(V_{L}^{\otimes 2}\right) \subset F_{4+2+\cdots+2}\left(V_{L}^{\otimes 2}\right)=V_{L}^{\otimes 2} .
$$

Let $\tau$ be the transposition of factors on $V_{L}^{\otimes 2}$, as in the proof of Proposition 6.4. It is clear that $\tau$ acts as the identity on $F_{2+\cdots+2}$ since this space is spanned by graphs which are unions of 2-cycles. On the other hand, Lemma 6.3 shows that $\tau$ acts by -1 on $F_{4+2+\cdots+2} / F_{2+\cdots+2}$. From this it follows that $\operatorname{Sym}^{2}\left(V_{L}\right)=F_{2+\cdots+2}=\operatorname{gr}_{2+\cdots+2}$ and that the quotient map $V_{L}^{\otimes 2} \rightarrow \mathrm{gr}_{4+2+\cdots+2}$ factors to give an isomorphism $\bigwedge^{2} V_{L} \rightarrow$ $\mathrm{gr}_{4+2+\cdots+2}$.

\subsection{The action of $\mathfrak{S}_{L}$ on degree two spaces}

Having described the spaces $\operatorname{gr}_{p}\left(V_{L}^{\otimes 2}\right)$, we now turn to their structure as $\mathfrak{S}_{L}$-modules. Our main result is the following. Note that characteristic 0 notions and arguments from the representation theory of $\mathfrak{S}_{L}$ make sense over $\mathbb{Z}[1 /|L|$ !] with the obvious changes.

Proposition 6.5. In the following table, each $\mathfrak{S}_{L}$-module is multiplicity-free. The set of irreducibles it contains corresponds to the given set of partitions.

\begin{tabular}{c|c}
\hline $\mathfrak{S}_{L}$-module & Set of partitions of $|L|$ \\
\hline $\operatorname{Sym}^{2}\left(V_{L}\right)$ & at most four parts, all even \\
$\wedge^{2} V_{L}$ & exactly four parts, all odd \\
$V_{L}^{\otimes 2}$ & union of previous two sets \\
$R_{L}^{(2)}$ & at most three parts, all even \\
$I_{L}^{(2)}$ & exactly four parts, all even \\
\hline
\end{tabular}

These statements hold over $\mathbb{Z}[1 /|L|$ !]. 
For instance, $I_{L}^{(2)}$ is a direct sum of those irreducible representations of $\mathfrak{S}_{L}$ corresponding to partitions of $|L|$ into exactly four even parts. To prove the proposition it suffices to work over the complex numbers $\mathbb{C}$. We use Schur-Weyl theory: for a vector space $A$ we have a decomposition

$$
A^{\otimes L} \cong \bigoplus_{\lambda} M_{\lambda} \otimes \mathbf{S}_{\lambda}(A)
$$

where the sum is over all partitions $\lambda$ of $|L|, M_{\lambda}$ denotes the irreducible representation of $\mathfrak{S}_{L}$ attached to a partition $\lambda$, and $\mathbf{S}_{\lambda}$ is the Schur functor corresponding to $\lambda$. Here $A^{\otimes L}$ denotes the tensor product of copies of $A$ indexed by $L$. The above decomposition respects the action of $\mathfrak{S}_{L}$ and is functorial with respect to $A$.

Let $P$ be the two-dimensional vector space over $\mathbb{C}$ with basis $\{x, y\}$. Let $\bigwedge^{2} P \rightarrow \mathbb{C}$ be the isomorphism taking $x \wedge y$ to 1 . We define $\mathrm{SL}(P)=\operatorname{Sp}(P)$ to be the group of linear transformations of $P$ preserving this alternating form.

Since $V_{L}$ is the space of degree one invariants, it is equal, by definition, to $\left(P^{\otimes L}\right)^{\mathrm{SL}(P)}$. The action of $\mathfrak{S}_{L}$ on $V_{L}$ in this description is the obvious one. We thus have an $\mathfrak{S}_{L^{-}}$ equivariant isomorphism

$$
V_{L} \otimes V_{L}=\left(P^{\otimes L} \otimes P^{\otimes L}\right)^{\mathrm{SL}(P) \times \mathrm{SL}(P)}=\left((P \otimes P)^{\otimes L}\right)^{\mathrm{SL}(P) \times \operatorname{SL}(P)} .
$$

The $\mathfrak{S}_{L}$-action is the diagonal action on the first two spaces and the usual one on the last space. The above isomorphism is also equivariant with respect to the transposition of factors $\tau$. We now apply the Schur-Weyl decomposition to obtain

$$
V_{L} \otimes V_{L} \cong \bigoplus_{\lambda} M_{\lambda} \otimes \mathbf{S}_{\lambda}(P \otimes P)^{\mathrm{SL}(P) \times \mathrm{SL}(P)} .
$$

The space $P \otimes P$ has a natural symmetric inner product coming from the alternating inner product on $P$. This inner product is preserved by the group $\mathrm{SL}(P) \times \mathrm{SL}(P)$, and it is not hard to see that the resulting map $\mathrm{SL}(P) \times \mathrm{SL}(P) \rightarrow \mathrm{SO}(P \otimes P)$ is surjective. The transposition $\tau$ on $P \otimes P$ preserves the inner product but has determinant -1 . Thus $\tau$ and $\mathrm{SO}(P \otimes P)$ generate $\mathrm{O}(P \otimes P)$. Now, the space $\operatorname{Sym}^{2}\left(V_{L}\right)$ is just the $\tau$-invariant part of $V_{L} \otimes V_{L}$, so

$$
\operatorname{Sym}^{2}\left(V_{L}\right) \cong \bigoplus_{\lambda} M_{\lambda} \otimes \mathbf{S}_{\lambda}(P \otimes P)^{\mathrm{O}(P \otimes P)} .
$$

Similarly, $\bigwedge^{2} V_{L}$ is just the subspace of $V_{L} \otimes V_{L}$ on which $\tau$ acts by -1 and so

$$
\bigwedge^{2} V_{L} \cong \bigoplus_{\lambda} M_{\lambda} \otimes \mathbf{S}_{\lambda}(P \otimes P)^{\mathrm{O}(P \otimes P),-}
$$

where the minus sign means to take the subspace on which $\mathrm{O}(P \otimes P)$ acts by its sign representation. The first three lines of the table in Proposition 6.5 now follow from the $n=4$ case of the following lemma. This lemma appears as statements (1) and (2) in the proof of [Ku, Lemma 2.2].

Lemma 6.6 (S. Kumar). Let $\lambda$ be a partition and let $V$ be a vector space of dimension $n$ with a non-degenerate symmetric inner product.

- $\mathbf{S}_{\lambda}(V)^{\mathrm{O}(V)}$ is one-dimensional if $\lambda$ has at most $n$ parts, all even, and is zero otherwise.

- $\mathbf{S}_{\lambda}(V)^{\mathrm{O}(V),-}$ is one-dimensional if $\lambda$ has exactly $n$ parts, all odd, and is zero otherwise. 
We now turn our attention to the space $R_{L}^{(2)}$, the degree two invariants. We have an $\mathfrak{S}_{L^{-}}$ equivariant isomorphism $R_{L}^{(2)}=\left(\operatorname{Sym}^{2}(P)^{\otimes L}\right)^{\mathrm{SL}(P)}$, so

$$
R_{L}^{(2)} \cong \bigoplus_{\lambda} M_{\lambda} \otimes \mathbf{S}_{\lambda}\left(\operatorname{Sym}^{2}(P)\right)^{\mathrm{SL}(P)} .
$$

Use the alternating inner product on $P$ to define a symmetric inner product on $\operatorname{Sym}^{2}(P)$ via

$$
\left\langle v v^{\prime}, w w^{\prime}\right\rangle=\langle v, w\rangle\left\langle v^{\prime}, w^{\prime}\right\rangle+\left\langle v, w^{\prime}\right\rangle\left\langle v^{\prime}, w\right\rangle .
$$

The group $\operatorname{SL}(P)$ preserves this inner product and it is not hard to show that the map $\mathrm{SL}(P) \rightarrow \mathrm{SO}\left(\operatorname{Sym}^{2}(P)\right)$ is surjective. We thus have

$$
R_{L}^{(2)}=\bigoplus_{\lambda} M_{\lambda} \otimes \mathbf{S}_{\lambda}\left(\operatorname{Sym}^{2}(P)\right)^{\operatorname{SO}\left(\operatorname{Sym}^{2}(P)\right)} .
$$

The fourth line of the table in Proposition 6.5 now follows from the following lemma:

Lemma 6.7. Let $\lambda$ be a partition of an even number and let $V$ be a three-dimensional vector space with a non-degenerate symmetric inner product. Then $\mathbf{S}_{\lambda}(V)^{\mathrm{SO}(V)}$ is onedimensional if $\lambda$ has at most three parts, all of which are even, and is zero otherwise.

Proof. By the second part of Lemma 6.6 we have $\mathbf{S}_{\lambda}(V)^{\mathrm{O}(V),-}=0$, as three odd numbers cannot have an even sum. We thus have $\mathbf{S}_{\lambda}(V)^{\mathrm{SO}(V)}=\mathbf{S}_{\lambda}(V)^{\mathrm{O}(V)}$ and the result follows from the first part of Lemma 6.6.

The final line of the table in Proposition 6.5 follows from $R_{L}^{(2)} \cong \operatorname{Sym}^{2}\left(V_{L}\right) / I_{L}^{(2)}$.

\subsection{Degree three spaces}

We now turn our attention to the cubic spaces $V_{L}^{\otimes 3}$ and $\operatorname{Sym}^{3}\left(V_{L}\right)$. We say that a 3regular graph on $L$ is a benzene cycle if it is a cycle in which the edges alternate between being single and doubled (Figure 8 ). We use this term because molecules of benzene are depicted with such graphs (on six points), as in the figure. We use the term benzene chain for a chain of edges which alternate between being single and doubled. A benzene 2-cycle is interpreted to mean a triple edge. The main result of this section is the following:

Proposition 6.8. The space $\operatorname{Sym}^{3}\left(V_{L}\right)$ is spanned over $\mathbb{Z}[1 / 2]$ by graphs which are unions of benzene 2-, 4- and 6-cycles. In particular, $\operatorname{gr}_{p}\left(\operatorname{Sym}^{3}\left(V_{L}\right)\right)=0$ unless the parts of $p$ are at most 6 .

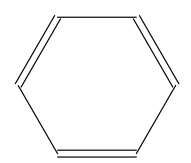

Fig. 8. A benzene 6-cycle.

We deduce Proposition 6.8 from the following:

Proposition 6.9. Let $L$ be a set of cardinality at least 8 and let $\Delta$ be a fixed matching on $L$. Then $V_{L}$ is spanned by those $Y_{\Gamma}$ for which the graph $\Delta \Gamma$ is not connected. 
Proof. Let $\Gamma$ be a given matching. Think of $\Delta$ as having dotted edges and $\Gamma$ as having dark edges, so $\Delta \Gamma$ is a regular 2-colored graph. We must show that by using only dark Plücker relations we can write it as a sum of disconnected graphs. (We assume that $\Delta \Gamma$ is connected to begin with.)

Let $a, b, c$ and $d$ be four consecutive vertices in $\Delta \Gamma$, where $\overline{a b}$ is a dark edge. We have the dark Plücker relation

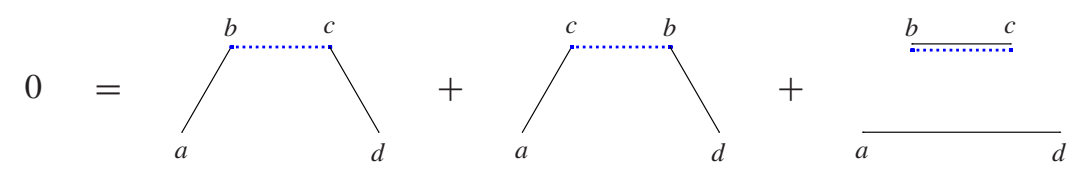

The rightmost graph is disconnected. Thus, working modulo such graphs, we may transpose dotted edges at the cost of a sign. Now let $a, b, c, d, e$ and $f$ be six consecutive vertices where $\overline{a b}$ is a dark edge. We have the dark Plücker relation

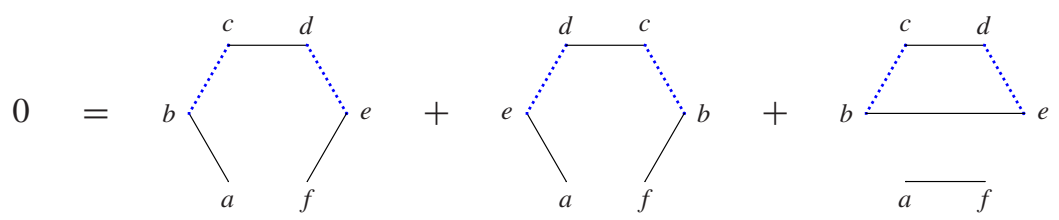

As before, the rightmost graph is disconnected. This shows that we may take four consecutive vertices and reverse their direction, at the cost of a sign, assuming the outer two edges are dotted. In the same way, by considering eight consecutive vertices we see that a consecutive string of six vertices may be flipped at the cost of a sign, assuming that the outer two edges are dotted.

Now let $a, b, c, d, e$ and $f$ be six consecutive vertices, the outer two edges of which are dotted. We write $[a, b, c, d, e, f]$ to denote this situation. By the above, we have

$$
\begin{aligned}
{[a, b, c, d, e, f] } & =-[f, e, d, c, b, a]=[f, e, a, b, c, d] \\
& =-[b, a, e, f, c, d]=[b, a, d, c, f, e]=-[a, b, c, d, e, f]
\end{aligned}
$$

so $Y_{\Gamma}=-Y_{\Gamma}$ modulo matchings for which $\Delta \Gamma$ is disconnected, establishing the proposition.

By keeping track of the discarded graphs in the above proof we obtain a complicated identity, shown in Figure 9, that we will use on a few later occasions. As an immediate corollary of the above proposition we have the following.

Corollary 6.10. Let $\Gamma$ be a regular 3-colored graph and let $\Phi$ be a benzene cycle in $\Gamma$. Then in $V_{L}^{\otimes 3}$ one can write $Y_{\Gamma}$ as a linear combination of $Y_{\Gamma_{i}}$ 's where in each $\Gamma_{i}$ the subgraph $\Phi$ is replaced with a union of benzene 2-, 4- and 6-cycles. This holds over $\mathbb{Z}[1 / 2]$.

Proof. Assume $\Phi$ has at least eight vertices (otherwise there is nothing to prove). Let $\Delta$ be the doubled edges of $\Phi$ and let $\Phi^{\prime}$ be the single edges of $\Phi$, so that $\Phi=\Delta \Phi^{\prime}$. Use Proposition 6.9 to rewrite $Y_{\Phi^{\prime}}$ as a sum of $Y_{\Phi_{i}^{\prime}}$ with $\Delta \Phi_{i}^{\prime}$ disconnected. This will 


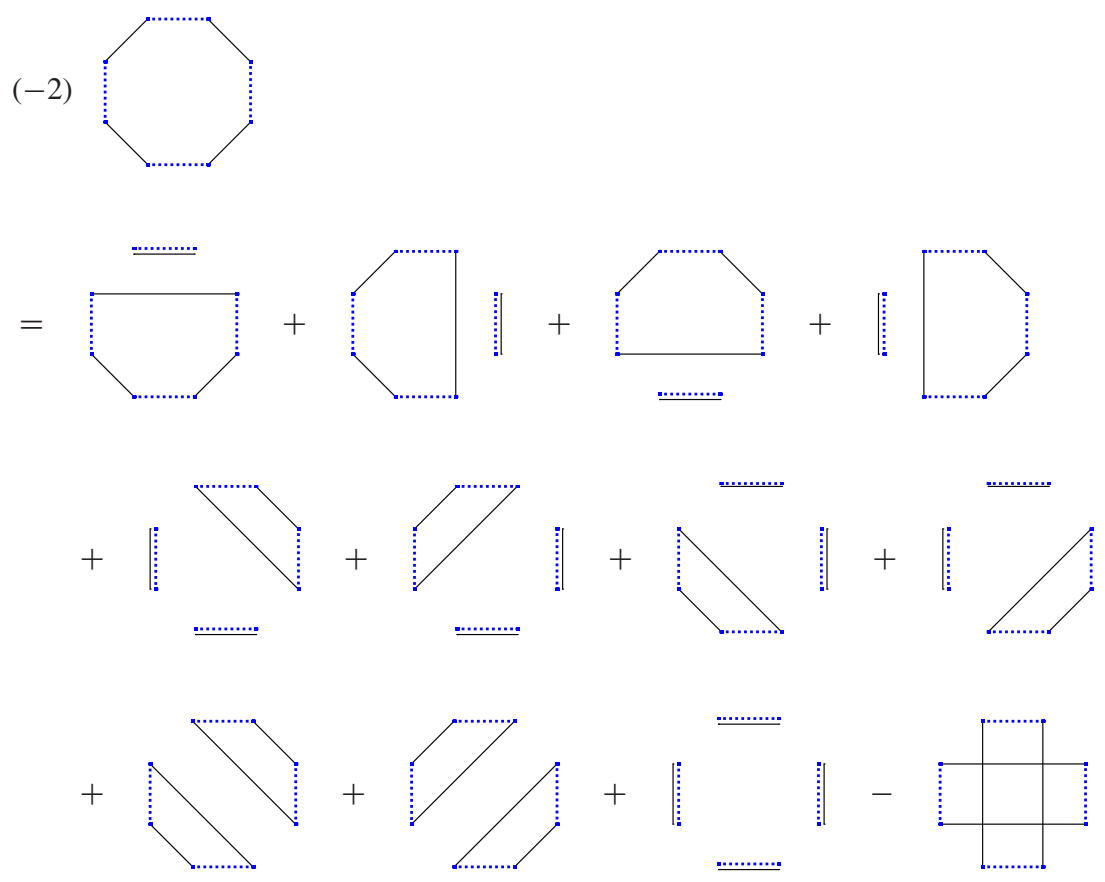

Fig. 9. A graphical identity. The dotted edges here are not relevant to the identity: they are just drawn to emphasize the disconnectedness of the graphs on the right side. Thus this is a linear relation between $Y_{\Gamma}$ 's where the $\Gamma$ are matchings on eight points. This identity can be obtained by applying the procedure of Proposition 6.9 to the term on the left side, or by applying the straightening algorithm to the final term on the right side.

rewrite $Y_{\Gamma}$ as a sum of $Y_{\Gamma_{i}}$ where in each $Y_{\Gamma_{i}}$ the benzene cycle $\Phi$ has been replaced with a union of smaller benzene cycles. Continuing in this way, one deduces the statement.

We can now prove Proposition 6.8.

Proof of Proposition 6.8. Let $\Gamma$ be a regular 3-colored graph on $L$, thought of as having colors dashed, solid and dotted. Consider the dashed-solid subgraph of $\Gamma$. We may apply Proposition 6.1 to rewrite this graph as a sum of graphs which are unions of 2-cycles. Thus we may as well assume that the dashed-solid subgraph of $\Gamma$ is made up of 2-cycles. By now considering the dotted edges as well, we see that $\Gamma$ is a union of benzene cycles. We may now appeal to Corollary 6.10 to break up large benzene cycles into smaller ones.

\subsection{The action of $\mathfrak{S}_{L}$ on degree three spaces}

We now examine the spaces $\operatorname{gr}_{p}\left(\operatorname{Sym}^{3}\left(V_{L}\right)\right)$ more closely when $L$ is small and determine their structure as $\mathfrak{S}_{L}$-modules. We assume throughout that $|L|$ ! is invertible. We denote by $M_{L}$ the $\mathbb{Z}\left[\mathfrak{S}_{L}\right]$-module with a basis given by the set of undirected matchings on $L$. 
Proposition 6.11. As an $\mathfrak{S}_{L}$-representation the space $M_{L}$ is multiplicity-free and contains those irreducibles corresponding to partitions of $|L|$ into even parts.

This proposition is well-known and essentially equivalent to the decomposition of the plethysm $\operatorname{Sym}^{n} \mathrm{Sym}^{2}$. In any case, we only need to use this when $L$ has cardinality four or six, where it can easily be established by hand. We now begin our study of $\operatorname{gr}_{p}\left(\operatorname{Sym}^{3}\left(V_{L}\right)\right)$. For the sake of brevity, we will denote this space simply by $\mathrm{gr}_{p}$ in this section. We will also write $\mathfrak{S}_{n}$ in place of $\mathfrak{S}_{L}$ where $n=|L|$. Our first result is the following:

Proposition 6.12. The space $\mathrm{gr}_{2+\ldots+2}$ is multiplicity-free and contains only those irreducible representations of $\mathfrak{S}_{n}$ which have an even number of parts.

Proof. The space $\mathrm{gr}_{2+\cdots+2}$ is the image of the map $\epsilon \otimes M_{L} \rightarrow \operatorname{Sym}^{3}\left(V_{L}\right)$ which takes a matching $\Gamma$ to $Y_{\Gamma}^{3}$. The result now follows from Proposition 6.11 .

We will only need to use the above proposition for $n \leq 6$. We now determine the spaces $\operatorname{gr}_{p}$ completely for $n=4$ and 6 . As a warm-up, consider the $n=2$ case: $\mathrm{gr}_{2}$ is onedimensional, and it is the sign representation of $\mathfrak{S}_{2}$. Reason: the space $\operatorname{Sym}^{3}\left(V_{L}\right)$ is spanned by $Y_{\Gamma}$ where $\Gamma$ is the graph on $L$ with a tripled edge. The group $\mathfrak{S}_{2}$ fixes $\Gamma$ and so acts on $Y_{\Gamma}$ through the sign character. All other $\operatorname{gr}_{p}$ in this case are zero. We now turn to $n=4$.

Proposition 6.13. (a) The space $\mathrm{gr}_{2,2}$ is free over $\mathbb{Z}[1 / 4$ ! ] and three-dimensional. As an $\mathfrak{S}_{4}$-representation it decomposes into two irreducibles corresponding to the partitions $2+2$ and $1+1+1+1$.

(b) The space $\mathrm{gr}_{4}$ is free over $\mathbb{Z}\left[1 / 4\right.$ !] and one-dimensional. The representation of $\mathfrak{S}_{4}$ on it is trivial.

Proof. The space $\mathrm{gr}_{4}$ is spanned by benzene 4-cycles. We have the identity

$$
0=1 \mid
$$

obtained by Plückering the dotted edges. We thus see that if $\Gamma$ is benzene 4-cycle in which $\overline{a b}$ is a doubled edge then $Y_{\Gamma}+Y_{(a b) \Gamma}=0$ in $\operatorname{gr}_{4}$, which can be rephrased as $(a b) Y_{\Gamma}=Y_{\Gamma}$. In particular, two benzene 4-cycles are equal in $\mathrm{gr}_{4}$ (up to a possible sign) if they have the same doubled edges. By cubing

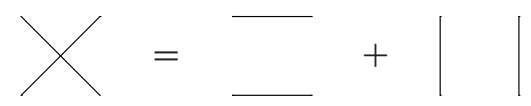

we obtain

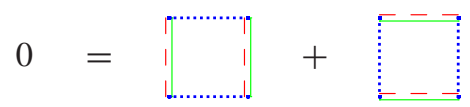

in $\mathrm{gr}_{4}$ (all other terms belong to $F_{2,2}$ ). This shows that we can switch which edges are the doubled edges. We have thus shown that $\mathrm{gr}_{4}$ is one-dimensional. Since we already know that some transpositions act by the identity, it follows that $\mathfrak{S}_{4}$ acts trivially on $\mathrm{gr}_{4}$. 
Now, $\operatorname{Sym}^{3}\left(V_{L}\right)$ is four-dimensional. We know that $\mathrm{gr}_{4}$ is one-dimensional and so $\mathrm{gr}_{2,2}$ must be three-dimensional. By Proposition 6.12 we know that $\operatorname{gr}_{2,2}$ is a quotient of the direct sum of the irreducible representations of $\mathfrak{S}_{4}$ corresponding to $2+2$ and $1+1+1+1$. As this direct sum has dimension 3 , it follows that $\mathrm{gr}_{2,2}$ must equal it.

Finally we consider the case $n=6$. As stated in $\S 1.6$, the computer calculations in the proof are very mild and could probably be done by hand in a matter of hours.

Proposition 6.14. (a) The space $\operatorname{gr}_{2,2,2}$ is free over $\mathbb{Z}[1 / 6$ !] and 15 -dimensional. As an $\mathfrak{S}_{6}$-representation it decomposes into three irreducibles, corresponding to the partitions $3+3,2+2+1+1$ and $1+1+1+1+1+1$.

(b) The space $\mathrm{gr}_{4,2}$ is free over $\mathbb{Z}[1 / 6$ !], 15-dimensional and decomposes into two irreducibles: one corresponding to $5+1$ and the other to $4+1+1$.

(c) The space $\operatorname{gr}_{6}$ is free over $\mathbb{Z}[1 / 6$ ! ] and five-dimensional. It is irreducible and corresponds to $3+3$.

(d) Let $Q$ be a set of cardinality six and let $c, d$ and e be three distinct elements of $Q$. Then $\mathrm{gr}_{6}\left(\operatorname{Sym}^{3}\left(V_{Q}\right)\right)$ is spanned by benzene 6-cycles in which cd or ce appears as a doubled edge. (We call the set $Q$ here, rather than $L$, since that is what it will be called in the one place where we apply this statement.)

Proof. The code for the computer calculations required here can be found on the webpage [HMSV5].

(a) A computer calculation shows that $F_{2,2,2}=\mathrm{gr}_{2,2,2}$ is 15-dimensional. By Proposition 6.12, the space $\mathrm{gr}_{2,2,2}$ is a quotient of the direct sum of the irreducible representations of $\mathfrak{S}_{6}$ corresponding to the partitions given in the statement of this proposition. Since this direct sum is also 15-dimensional the quotient map is an isomorphism.

(b) A computer calculation shows that $F_{4,2}$ is 30-dimensional, and so $\mathrm{gr}_{4,2}=$ $F_{4,2} / F_{2,2,2}$ is 15 -dimensional. Now observe that there is a map $F_{4} \otimes F_{2} \rightarrow F_{4,2}$ which takes an element of $F_{4}$ on the vertices $\{1,2,3,4\}$ and appends a tripled edge on the vertices $\{5,6\}$. One sees using this that that $\operatorname{gr}_{4,2}$ is a quotient of $\operatorname{Ind}_{\mathfrak{S}_{4} \times \mathfrak{S}_{2}}^{\mathfrak{S}_{6}}\left(\mathrm{gr}_{4} \otimes \mathrm{gr}_{2}\right)$. By the Littlewood-Richardson rule, the induction is a direct sum of the two irreducible representations of $\mathfrak{S}_{6}$ corresponding to the partitions given in the statement of the proposition. Since this sum is also 15-dimensional, the quotient map is an isomorphism.

(c) On the one hand, we have a non-canonical $\mathfrak{S}_{6}$-equivariant decomposition $\operatorname{Sym}^{3}\left(V_{L}\right) \cong \operatorname{gr}_{2,2,2} \oplus \mathrm{gr}_{4,2} \oplus \mathrm{gr}_{6}$. On the other hand, a character computation shows that $\operatorname{Sym}^{3}\left(V_{L}\right) \cong \mathrm{gr}_{2,2,2} \oplus \mathrm{gr}_{4,2} \oplus M_{3+3}$ (where $M_{3,3}$ is the irreducible representation corresponding to $3+3)$. Thus $\mathrm{gr}_{6}=M_{3+3}$.

(d) This is a straightforward computer calculation.

\section{Retrogeneration of the ideal}

In this section, we prove that for $|L|$ sufficiently large, the ideal of relations is retrogenerated (generated by quadratics and relations on fewer points): 
Theorem 7.1. Let $L$ be an even set of cardinality at least 10 . Then $I_{L}=I_{L}^{\text {retro }}$. For $|L| \geq 12$ this holds over $\mathbb{Z}[1 / 2]$ while for $|L|=10$ it holds over $\mathbb{Z}[1 / 10$ !].

The ideal $I_{L}^{\text {retro }}$ of retrogenerated relations will be defined in $\S 7.1$. We use general structural arguments to prove Theorem 7.1 when $|L| \geq 12$ but when $|L|=10$ the small size of the graphs involved forces us to give an inelegant ad hoc argument. We suggest that a reader consider skipping the arguments in the $|L|=10$ case on a first reading.

\subsection{Outer multiplication and the retrogenerated ideal $I_{L}^{\text {retro }}$}

Let $L$ and $L^{\prime}$ be even sets. We have an outer multiplication map

$$
\otimes: V_{L}^{\otimes n} \otimes V_{L^{\prime}}^{\otimes n} \rightarrow V_{L \amalg L^{\prime}}^{\otimes n}, \quad Y_{\Gamma} \otimes Y_{\Gamma^{\prime}} \mapsto Y_{\Gamma \amalg \Gamma^{\prime}} .
$$

In words, one takes a colored graph $\Gamma$ on $L$ and a colored graph $\Gamma^{\prime}$ on $L^{\prime}$, with the same set of colors, and obtains a colored graph on $L \amalg L^{\prime}$ by taking the disjoint union of $\Gamma$ and $\Gamma^{\prime}$. We will often omit the symbol $\otimes$ and write the outer product using juxtaposition.

Outer multiplication does not descend to symmetric powers, as shown by the following example:
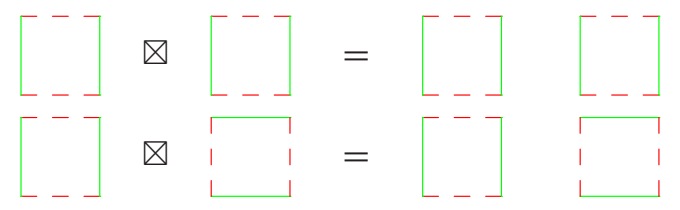

Let $L$ and $L^{\prime}$ be the two sets of four vertices occurring on the left sides. The two left sides above define equal elements of $\operatorname{Sym}^{2}\left(V_{L}\right) \otimes \operatorname{Sym}^{2}\left(V_{L^{\prime}}\right)$. However, the two right sides are different elements of $\operatorname{Sym}^{2}\left(V_{L} \amalg L^{\prime}\right)$ - their difference is the simplest binomial relation (1.4).

One sees from the above example that outer multiplication does not descend to symmetric powers for the following reason: if $\Gamma$ is an $n$-colored graph on $L$ and one permutes the colors in each connected component of $\Gamma$ to obtain a new $n$-colored graph $\Gamma^{\prime}$ then $Y_{\Gamma}$ and $Y_{\Gamma^{\prime}}$ do not represent the same element of $\operatorname{Sym}^{n}\left(V_{L}\right)$ in general. Now, if $\Gamma$ and $\Gamma^{\prime}$ are as in the previous sentence then $Y_{\Gamma}-Y_{\Gamma^{\prime}}$ lies in the ideal $Q_{L}$ of $\operatorname{Sym}\left(V_{L}\right)$ generated by quadratic relations. (Reason: any permutation of colors can be obtained by successive transpositions of colors, and the relations thus arising are clearly quadratic.) We hence find that outer multiplication descends to a map

$$
\otimes: \operatorname{Sym}^{n}\left(V_{L}\right) / Q_{L}^{(n)} \otimes \operatorname{Sym}^{n}\left(V_{L^{\prime}}\right) / Q_{L^{\prime}}^{(n)} \rightarrow \operatorname{Sym}^{n}\left(V_{L \amalg L^{\prime}}\right) / Q_{L \amalg L^{\prime}}^{(n)}
$$

and that $I_{L}^{(n)} / Q_{L}^{(n)} \otimes \operatorname{Sym}^{n}\left(V_{L^{\prime}}\right) / Q_{L^{\prime}}^{(n)}$ is mapped into $I_{L \amalg L^{\prime}}^{(n)} / Q_{L \amalg L^{\prime}}^{(n)}$ under $\otimes$, that is, the outer product of anything with a relation is still a relation. A motivating example appeared in the introduction: the Segre relation on six points (1.2) induces a relation on eight points (1.3).

For a given $L$, we define the ideal of retrogenerated relations, denoted $I_{L}^{\text {retro }}$, to be the ideal of $\operatorname{Sym}\left(V_{L}\right) / Q_{L}$ generated by the images of $I_{L^{\prime}}^{(n)} / Q_{L^{\prime}}^{(n)} \otimes \operatorname{Sym}^{n}\left(V_{L^{\prime \prime}}\right) / Q_{L^{\prime \prime}}^{(n)}$ under 
$\otimes$ as $\left(L^{\prime}, L^{\prime \prime}\right)$ varies over all partitions of $L$ into two disjoint proper even subsets and $n$ varies over all positive integers. We also write $I_{L}^{\text {retro }}$ for the inverse image of $I_{L}^{\text {retro }}$ under $\operatorname{Sym}\left(V_{L}\right) \rightarrow \operatorname{Sym}\left(V_{L}\right) / Q_{L}$. We have inclusions $Q_{L} \subset I_{L}^{\text {retro }} \subset I_{L}$. (Theorem 8.1 will show that these three ideals are all the same.) We say a relation (in $I_{L}$ ) is retrogenerated if it lies in $I_{L}^{\text {retro }}$.

A basic fact is that outer multiplication does not increase the "essential degree" of a relation:

Proposition 7.2. Let $L=L^{\prime} \amalg L^{\prime \prime}$ be a partition of $L$ into two proper even subsets. Let $x \in I_{L^{\prime}}^{(n)} / Q_{L^{\prime}}^{(n)}$ belong to the ideal generated by relations of degree $\leq k$ and let $y \in \operatorname{Sym}^{n}\left(V_{L^{\prime \prime}}\right) / Q_{L^{\prime \prime}}^{(n)}$. Then the outer product $x \otimes y$ belongs to the ideal of $\operatorname{Sym}\left(V_{L}\right) / Q_{L}$ generated by relations of degree $\leq k$.

Proof. Write $x=\sum a_{i} r_{i}$ where $r_{i}$ is a relation of degree $\leq k$ and $a_{i}$ belongs to $\operatorname{Sym}\left(V_{L^{\prime}}\right) / Q_{L^{\prime}}$ and write $y=\sum y_{i}$ where each $y_{i}$ is a product of degree one elements. The outer product of $x \otimes y$ is a sum of terms of the form $a_{i} r_{i} \otimes y_{j}$. Say $r_{i}$ has degree $k^{\prime} \leq k$ so that $a_{i}$ has degree $n-k^{\prime}$, and write $y_{j}=b_{1} \cdots b_{n}$. Then $a_{i} r_{i} \otimes y_{j}=\left(a_{i} \otimes\left(b_{1} \cdots b_{n-k^{\prime}}\right)\right)\left(r_{i} \otimes\left(b_{n-k^{\prime}+1} \cdots b_{n}\right)\right)$ — this basic compatibility between outer multiplication and usual multiplication is trivial to verify. We have thus shown that $a_{i} r_{i} \otimes y_{j}$ is a multiple of $r_{i} \otimes\left(b_{n-k^{\prime}+1} \cdots b_{n}\right)$, a relation of degree $k^{\prime} \leq k$.

Outer multiplication, simple binomial relations and the retrogenerated ideal are quite formal constructions and are present when studying $X^{n} / / G$ for any $X$ and $G$. They are described nicely by the formalism of the third author mentioned in $\S 1.7$. By contrast, the following two propositions are specific to the present case.

Proposition 7.3. Let $\Sigma=(\Gamma, \mathscr{U})$ be a generalized Segre datum for which $\Gamma$ is disconnected. Then $\operatorname{Rel}(\Sigma)$ belongs to $I_{L}^{\text {retro }}$.

Proof. Write $\Gamma=\Gamma_{1} \amalg \Gamma_{2}$. If one of $\Gamma_{1}$ or $\Gamma_{2}$ is entirely contained within one of the parts $U_{R}, U_{G}$ or $U_{B}$ then the relation $\operatorname{Rel}(\Sigma)$ is manifestly retrogenerated. For instance, if $\Gamma_{1}$ is contained within one of the parts then $\Gamma_{2}$ with the partition induced from $\mathscr{U}$ forms a generalized Segre datum $\Sigma_{2}$ and $\operatorname{Rel}(\Sigma)$ is the outer product of $Y_{\Gamma_{1}}$ and $\operatorname{Rel}\left(\Sigma_{2}\right)$. If neither $\Gamma_{1}$ nor $\Gamma_{2}$ is contained solely within one part then each contains a pair of special edges and the datum $\Sigma$ is forced to be degenerate (of the second case given in $\$ 5.4$ ). The relation $\operatorname{Rel}(\Sigma)$ thus belongs to $Q_{L} \subset I_{L}^{\text {retro }}$ by Proposition 5.2.

The next proposition is a key point in our inductive arguments.

Proposition 7.4. The ideal $I_{L}^{\text {retro }} \subset \operatorname{Sym}\left(V_{L}\right) / Q_{L}$ is generated over $\mathbb{Z}[1 / 2]$ by the images of $I_{L^{\prime}}^{(3)} / Q_{L^{\prime}}^{(3)} \otimes \operatorname{Sym}^{3}\left(V_{L^{\prime \prime}}\right) / Q_{L^{\prime \prime}}^{(3)}$ under $\otimes$ as $\left(L^{\prime}, L^{\prime \prime}\right)$ varies over all partitions of $L$ into two disjoint subsets where $L^{\prime \prime}$ has cardinality 2 , 4 or 6.

Proof. Proposition 7.2 shows that $I_{L}^{\text {retro }}$ is generated by elements of the form $r \otimes Y_{\Gamma}$ where $r$ belongs to $I_{L^{\prime}}^{(3)} / Q_{L^{\prime}}^{(3)}$ and $Y_{\Gamma}$ belongs to $\operatorname{Sym}^{3}\left(V_{L^{\prime \prime}}\right) / Q_{L^{\prime \prime}}^{(3)}$, as $\left(L^{\prime}, L^{\prime \prime}\right)$ varies over all partitions of $L$ into two disjoint even subsets. Proposition 6.8 shows that we can 
write $Y_{\Gamma}=\sum a_{i} Y_{\Gamma_{i}}$ where $a_{i}$ belongs to $\mathbb{Z}[1 / 2]$ and each $\Gamma_{i}$ is a union of benzene 2-, 4- and 6-cycles. It follows that $Y_{\Gamma_{i}}$ is itself an outer product $Y_{\Gamma_{i 1}} \otimes \cdots \otimes Y_{\Gamma_{i n}}$ where each $Y_{\Gamma_{i j}}$ is a benzene 2-, 4- or 6-cycle. Since $\nabla$ is associative, we have $r \otimes Y_{\Gamma_{i}}=$ $\left(r \otimes Y_{\Gamma_{i 1}} \otimes \cdots \otimes Y_{\Gamma_{i(n-1)}}\right) \otimes Y_{\Gamma_{i n}}$, which expresses $r \otimes Y_{\Gamma_{i}}$ as the outer product of a graph on 2, 4 or 6 fewer points with a graph on 2, 4 or 6 points. Thus $r \otimes Y_{\Gamma}$ is a sum of such graphs, which establishes the proposition.

\subsection{Square rotation relations}

To prove Theorem 7.1 we introduce the square rotation relations, needed only in this proof. A square rotation datum is a pair $\Pi=(\Gamma, U)$ where $U$ is a subset of $L$ of cardinality 4 and $\Gamma$ is an undirected graph on $L$ with edges colored light and dark such that:

- The vertices of $L \backslash U$ have dark valence one and light valence two.

- The vertices of $U$ have dark valence zero and light valence one.

Let $\Pi$ be a given square rotation datum. Suppose that a pair of edges $e$ and $e^{\prime}$ in $\Gamma$ have the same color. Let $\Gamma+\Gamma^{\prime}+\Gamma^{\prime \prime}=0$ be the Plücker relation on $e$ and $e^{\prime}$. Then $\Pi^{\prime}=\left(\Gamma^{\prime}, U\right)$ and $\Pi^{\prime \prime}=\left(\Gamma^{\prime \prime}, U\right)$ are both square rotation data. We define the space of square rotation data to be the $\mathbb{Z}$-span of the square rotation data modulo the relations $\Pi+\Pi^{\prime}+\Pi^{\prime \prime}=0$.

Let $\Pi$ be a square rotation datum. We have the following quadratic relation on the four points in $U$ :

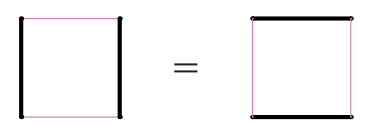

Multiplying both sides by $\Gamma$ we obtain an element of $R_{L}^{(1)} \otimes R_{L}^{(2)}$ which maps to zero in $R_{L}$. We may thus regard it as an element of $I_{L}^{(3)} / Q_{L}^{(3)}$, similar to what we did for generalized Segre relations. We call such relations square rotation relations. We have a linear map

$$
\text { Rel : }\{\text { the space of square rotation data }\} \rightarrow I_{L}^{(3)} / Q_{L}^{(3)}
$$

mapping a square rotation datum to its associated relation.

\subsection{Retrogeneration of square rotation relations}

The result is:

Proposition 7.5. If $L$ has cardinality at least 12 then any square rotation relation is retrogenerated.

We use a lemma to prove the proposition. Let $\Pi$ be a given square rotation datum. The light subgraph of $\Gamma$ has valence two everywhere except at the four vertices in $U$. It thus breaks up into a union of cycles and two paths terminating in $U$. We call these two paths the special paths. The length of a special path is the number of vertices it contains. 
Lemma 7.6. (a) A square rotation relation with both special paths of even length lies in $Q_{L}$.

(b) A square rotation relation with one special path of even length is quadratic.

Proof. (a) The way we obtain a square rotation relation from the square rotation datum is to simply append the graph $\Gamma$ to the relation (7.1). Now, on each side of (7.1) there are two light edges. Change the color of these edges to grey. The hypothesis on the lengths of the special paths says that we can color every other edge in the special paths grey (keeping the first and last edges light) so that each vertex belongs to one grey and one light edge. We may assume that the light cycles occurring in $\Gamma$ have even size (as we can force this using Plücker relations), so we can pick a light-grey alternating coloring of them. Thus we have factored the light subgraph of $\Gamma$ in such a way that all the light edges appearing in (7.1) have been colored grey. But now the relation is evidently essentially quadratic (i.e., belongs to $Q_{L}$ ) since it is taking place solely on the dark-grey graph.

(b) If one special path has even length and one has odd length then $\Gamma$ has an odd light cycle. We can thus Plücker the odd special path and the odd cycle so that both special paths have even length. We now use part (a).

We now prove Proposition 7.5.

Proof of Proposition 7.5. Let a square rotation datum be given. By the above lemma, we can assume that each of the special paths has odd length. Using arguments similar to those occurring in the proof of Proposition 6.1, we can force the special paths to have lengths three. Thus each special path contains a single vertex not belonging to $U$. Call these two vertices $x$ and $y$. We now use Proposition 6.1 to convert the light cycles in $\Gamma$ into 2-cycles. We thus have a benzene chain joining $x$ to $y$. The graph $\Gamma$ now looks like the following (with the possibility that there are some additional dark-light benzene cycles not pictured):

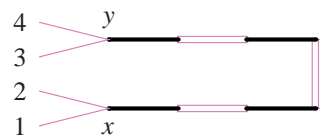

Here the numbered vertices constitute the set $U$. The special paths are 1-x-2 and 3-y-4. If there are in fact benzene cycles in $\Gamma$ then the relation is retrogenerated (the proof of this is similar to that of Proposition 7.3). We can thus assume that there are no benzene cycles and so the graph really does look like the above one. Since we have at least 12 vertices, the benzene chain will have at least four single dark edges, so we can apply Corollary 6.10 or the identity of Figure 9 to break up the benzene chain and get a disconnected graph. The associated relation will therefore be retrogenerated.

\subsection{Retrogeneration of the ideal on at least 12 points (Theorem 7.1 with $|L| \geq 12$ )}

We begin with two lemmas.

Lemma 7.7. Let $\Sigma=(\Gamma, \mathscr{U})$ be a generalized Segre datum, and let $U \subset U_{G}$ be a set of four vertices such that the dotted-solid graph of $\Gamma$ has a 4 -cycle contained in $U$. Let 
$\bar{\Gamma}$ be the graph obtained by rotating the colors in this 4-cycle and let $\bar{\Sigma}=(\bar{\Gamma}, \mathscr{U})$. Then $\operatorname{Rel}(\Sigma) \equiv \operatorname{Rel}(\bar{\Sigma})$ modulo quadratic and square rotation relations.

Proof. We must show that

$$
\operatorname{Rel}(\Sigma)-\operatorname{Rel}(\bar{\Sigma})=Y_{\Gamma}-Y_{\Gamma}^{\prime}-Y_{\bar{\Gamma}}+Y_{\bar{\Gamma}}^{\prime}
$$

belongs to the ideal generated by quadratic and square rotation relations. Clearly, $Y_{\Gamma}-$ $Y_{\bar{\Gamma}} \in Q_{L}$ since the dashed subgraph in each is the same, while $Y_{\Gamma}^{\prime}-Y_{\bar{\Gamma}}^{\prime}$ is a square rotation relation by definition.

Lemma 7.8. Let $\Sigma=(\Gamma, \mathscr{U})$ be a generalized Segre datum and let $U \subset U_{G}$ be a set of four vertices such that the dotted-solid graph of $\Gamma$ has a 4-cycle contained in $U$. Let $\left\{\Gamma_{i}\right\}$ be the three graphs obtained by replacing the 4-cycle on $U$ by two 2-cycles (there are three ways to do this). Let $\left\{\Sigma_{i}\right\}$ be the corresponding generalized Segre data. Then $2 \operatorname{Rel}(\Sigma) \equiv \sum \operatorname{Rel}\left(\Sigma_{i}\right)$ modulo quadratic and square rotation relations.

Proof. Recall the identity

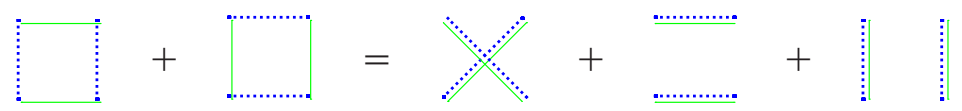

of the proof of Lemma 6.3. This identity holds in $V_{L}^{\otimes 2}$, that is, it follows from the colored Plücker relations. This shows that in the space of generalized Segre data, we have $\Sigma+$ $\bar{\Sigma}=\sum_{i=1}^{3} \Sigma_{i}$ where $\bar{\Sigma}$ was defined in the previous lemma. By the previous lemma, we have $\operatorname{Rel}(\Sigma)=\operatorname{Rel}(\bar{\Sigma})$ modulo quadratic and square rotation relations. This proves the current lemma.

We now complete the proof of Theorem 7.1 when $|L| \geq 12$.

Proof of Theorem 7.1 when $|L| \geq 12$. By Theorem 5.1, it suffices to show that small generalized Segre cubic relations are retrogenerated. Thus let $\Sigma$ be a given small generalized Segre datum. We assume without loss of generality that $U_{R}$ has cardinality two, and that $\Sigma$ is non-degenerate.

We begin by considering the dotted-solid subgraph of $U_{G}$. This is a union of cycles and a single chain going between the two special dotted edges. By using Proposition 6.1, or more accurately the identity of Figure 7, we can convert this graph into a union of 2-cycles and 4-cycles and make the chain have length three (so that the two special dotted edges are joined by a single solid edge). We can now use the above two lemmas to convert the 4-cycles into 2-cycles, modulo retrogenerated relations.

We have thus reduced to the case where the dotted-solid subgraph of $\Gamma$ in $U_{G}$ is made up of 2-cycles (except for the path of length three involving the two special dotted edges). We now consider the dashed edges. Except for the special dotted edges, the graph $\left.\Gamma\right|_{U_{G}}$ is a union of benzene chains with the dotted and solid edges being paired. There are two incoming special dashed edges and two dashed edges connected to the two special dotted edges. From each of these edges a benzene chain emanates which must terminate at one of the other edges. These four dashed edges are thus contained in two benzene chains. 
If the two special dashed edges are contained in the same benzene chain then the graph is degenerate and the relation lies in $Q_{L}$. It therefore suffices to consider the case where each special dashed edge connects to a special dotted edge via a benzene chain.

Now, we may run the entire argument given above inside $U_{B}$ as well. We thus conclude that $\left.\Gamma\right|_{U_{B}}$ is a union of benzene chains with the dotted and solid edges being paired. The two special dashed edges connect with the two special solid edges by benzene chains. The picture is thus something like:

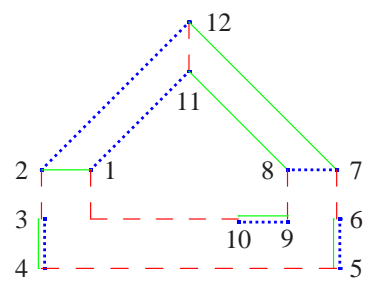

Here $U_{G}=\{1,2,3,4\}, U_{B}=\{5,6,7,8,9,10\}$ and $U_{R}=\{11,12\}$. If there are other benzene cycles present, then the relation is immediately retrogenerated. Also, the two benzene chains connecting the left and right side could be crossed; that is, the chains could go from 1 to 7 and 2 to 8 instead of as they do. However, one can always rectify this by Plückering two dashed edges such as $\overline{89}$ and $\overline{67}$ - one of the resulting graphs has the chains uncrossed while the other is degenerate. So the above graph is the only sort we need consider.

Now, we can repartition the vertices so that all the dotted-solid doubled edges are contained in $U_{G}$. For example, with the above graph we would repartition so that $U_{G}=$ $\{1,2,3,4,5,6,9,10\}, U_{B}=\{7,8\}$ and $U_{R}=\{11,12\}$. The resulting Segre datum yields the same relation as the original. We next point out that we can put all the dotted-solid doubled edges into a single benzene chain by Plückering two dashed edges. For example, in the above graph we would Plücker the edges $\overline{110}$ and $\overline{67}$. In one graph, $\overline{17}$ is a dashed edge and all the dotted-solid doubled edges are in a single benzene chain running between 2 and 8 . The other graph is degenerate. Now, since we have at least 12 vertices, the benzene chain will have at least four single dashed edges. It can therefore be broken apart using Corollary 6.10 or the identity of Figure 9, yielding a disconnected graph and therefore a retrogenerated relation. This completes the proof of Theorem 7.1 when $|L| \geq 12$.

\subsection{Retrogeneration of the ideal on 10-points (Theorem 7.1 with $|L|=10$ )}

For the remainder of $\S 7$ we let $L$ be a finite set of cardinality 10. To prove Theorem 7.1 in this case, we show the following:

Proposition 7.9. The space $I_{L}^{(3)} / I_{L}^{\mathrm{retro},(3)}$ has dimension at most two (over $\mathbb{Z}[1 / 2]$ ).

We now explain why this implies Theorem 7.1 for $|L|=10$. For the next few sentences we work over a field $k$ of characteristic not 2, 3, 5 or 7 . A character computation shows 
that $\operatorname{Sym}^{3}\left(V_{L}\right)$ does not contain the trivial or alternating representation. Now, the representation $I_{L}^{(3)} / I_{L}^{\text {retro,(3) }}$ is at most two-dimensional, and a summand of $\operatorname{Sym}^{3}\left(V_{L}\right)$. As any representation of $\mathfrak{S}_{L}$ of dimension at most two is made up of some combination of the trivial and alternating representations, it follows that $I_{L}^{(3)} / I_{L}^{\text {retro,(3) }}$ vanishes. Thus $I_{L}^{(3)}=I_{L}^{\text {retro,(3) }}$ over $k$. Since this holds for all $k$ of characteristic not 2, 3, 5 or 7, we conclude $I_{L}^{(3)}=I_{L}^{\text {retro,(3) }}$ over $\mathbb{Z}\left[1 / 10\right.$ !]. Since $I_{L}$ is generated by quadratics and $I_{L}^{(3)}$ (Theorem 5.1), this implies $I_{L}=I_{L}^{\text {retro }}$ over $\mathbb{Z}[1 / 10$ !].

We now turn to proving Proposition 7.9. We prove the following result:

Proposition 7.10. Let $\mathscr{U}=\left\{U_{G}, U_{R}, U_{B}\right\}$ be a fixed partition of $L$ with $\left|U_{R}\right|=2$. Consider the subspace $V$ of $I_{L}^{(3)} / I_{L}^{\text {retro,(3) }}$ spanned by all generalized Segre cubic relations coming from data with partition equal to $\mathscr{U}$. Then, over $\mathbb{Z}[1 / 2]$ :

(1) If $U_{G}$ (and thus $U_{B}$ ) has cardinality four then $V=0$.

(2) If either $U_{G}$ or $U_{B}$ has cardinality six then $\operatorname{dim} V \leq 1$.

We explain why Proposition 7.10 implies Proposition 7.9. Fix an order on L. Call a partition $\mathscr{U}=\left\{U_{G}, U_{R}, U_{B}\right\}$ of $L$ admissible if each part is non-empty of even cardinality, $U_{G}<U_{R}<U_{B}$ in the order and $\left|U_{R}\right|=2$. We call a generalized Segre cubic datum admissible if its partition is; we extend the notion to relations in the obvious manner. By inspection, there are three admissible partitions of $L$. One of these has $\left|U_{G}\right|=\left|U_{B}\right|=4$, while in the other two, one of $U_{G}$ or $U_{B}$ has cardinality six. It follows from Proposition 7.10 that the admissible Segre cubic relations span a subspace of $I_{L}^{(3)} / I_{L}^{\text {retro,(3) }}$ of dimension at most two. On the other hand, we know that the admissible generalized Segre cubic relations span $I_{L}^{(3)} / Q_{L}^{(3)}$ (see Remark 5.3). As $I_{L}^{\text {retro,(3) }}$ contains $Q_{L}^{(3)}$ we conclude that $I_{L}^{(3)} / I_{L}^{\text {retro,(3) }}$ has dimension at most two, establishing Proposition 7.9.

We now begin proving Proposition 7.10. We first consider the case where $\left|U_{G}\right|=4$ (so $\left|U_{B}\right|=4$ too). Consider the dotted-solid subgraph of $U_{G}$. In it there are two solid edges, two dotted edges contained entirely in $U_{G}$ and two dotted edges (the special ones) going between $U_{G}$ and $U_{R}$. By using the identity of Figure 7 we can force there to be a dotted-solid doubled edge. This implies that the special dotted edges are joined by one solid edge. By now considering the dashed edges, we see that there are two possibilities for the picture in $U_{G}$ :

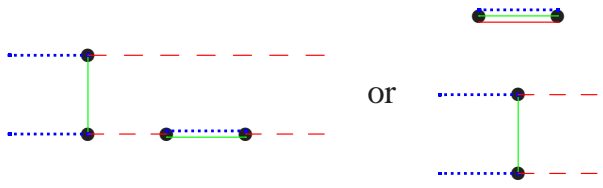

The right case is disconnected, hence retrogenerated. We thus need only consider the left case. We now go through the same considerations in $U_{B}$ as we just did in $U_{G}$ and conclude that it too must look like the graph on the left, except with the colors dotted and solid reversed. We find that the graph as a whole must be one of the two in the statement of the following lemma. That lemma then shows that that the generalized Segre relation we are considering is retrogenerated, which completes the $\left|U_{G}\right|=4$ case. 
Lemma 7.11. Let $\Gamma$ be one of the following two graphs:
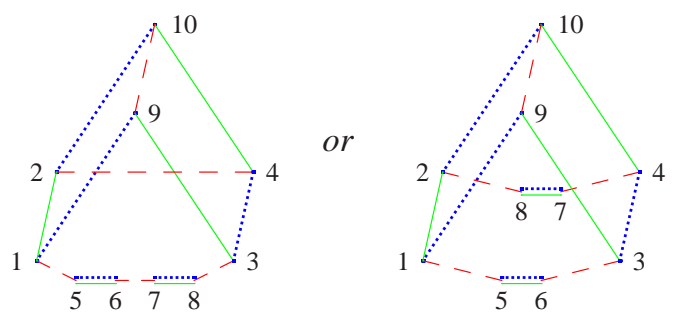

Let $\mathscr{U}=\left\{U_{R}, U_{G}, U_{B}\right\}$ be a partition such that $U_{R}=\{9,10\}, U_{G} \supset\{1,2\}, U_{B} \supset\{3,4\}$ and $\Sigma=(\Gamma, \mathscr{U})$ is a generalized Segre cubic datum. Then $\operatorname{Rel}(\Sigma)$ is retrogenerated over $\mathbb{Z}[1 / 2]$.

Proof. We first note that the relation $\operatorname{Rel}(\Sigma)$ is independent of the partition $\mathscr{U}$. We therefore assume that the vertices 5, 6, 7 and 8 belong to $U_{G}$. Now, let $\Gamma$ be the left graph. Redraw $\Gamma$ as follows:

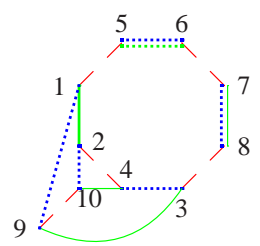

All the dashed edges other than $\overline{910}$ contain at least one vertex in $U_{G}$ so we are allowed to Plücker any of them together to obtain a relation of generalized Segre cubic data. We thus may apply the identity of Figure 9 to the dashed edges. All the resulting terms either come from fewer points by outer multiplication or else are degenerate. (Precisely: the first two graphs from the first row of Figure 9 come from fewer points, and the last two are degenerate. In the second row, all are degenerate except for the third. In the third row, all are degenerate except the first.) We conclude that $\operatorname{Rel}(\Sigma)$ is retrogenerated.

Now let $\Gamma$ be the right graph. By applying the Plücker relation to the edges $\overline{28}$ and $\overline{36}$ we get two graphs, one of which is degenerate, the other of which looks like the graph handled in the previous paragraph. Thus $\operatorname{Rel}(\Sigma)$ for the right graph is retrogenerated as well.

We now establish the second case of Proposition 7.10. Thus let $\mathscr{U}$ be a partition in which $\left|U_{G}\right|=6$. In this case, both $U_{R}$ and $U_{B}$ have cardinality two and so the situation is symmetric with respect to these two colors. We begin with the following observation:

Lemma 7.12. If there is a solid-dotted or solid-dashed doubled edge in $U_{G}$ then $\operatorname{Rel}(\Sigma)$ is retrogenerated over $\mathbb{Z}[1 / 2]$.

Proof. Say there is a solid-dotted doubled edge in $U_{G}$. Then, as in the case $\left|U_{G}\right|=4$, we can apply identity of Figure 7 to force there to be a second solid-dotted doubled edge in $U_{G}$. The graph $\Gamma$ must now look like one of the two in Lemma 7.11 and so, by that lemma, we obtain the present one. 
Now consider a general generalized Segre cubic datum $\Sigma$ with $\left|U_{G}\right|=6$. By applying the identity of Figure 7 repeatedly, or appealing to Proposition 6.1, we can arrange it so that the dotted-solid subgraph on $U_{G}$ is of the form

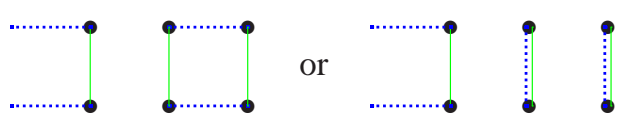

The right graph has a dotted-solid doubled edge, and so the associated relation belongs to $I_{L}^{\prime}$ by Lemma 7.12. We therefore need only consider the left graph. We now consider the dashed edges. There are two that are completely contained in $U_{G}$ and the two special edges, each of which has one vertex in $U_{G}$. Here are three possible graphs:
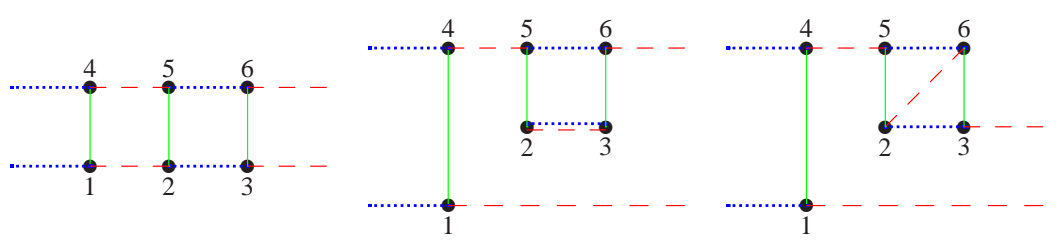

We will refer to these as type A, B and C, respectively. Of course, there are many other possibilities for what the graph could look like. However, all reduce to one of the above three types after some simple Plücker relations. For instance, one could consider the graph which is like the type A one, but where the dotted and solid colors are switched in the square 2-3-5-6. By applying the Plücker relation to the edges $\overline{45}$ and the dashed special edge containing 3 , this relation is rewritten as a sum of two of those appearing above.

We now have the following result:

Lemma 7.13. Type $B$ and $C$ relations are retrogenerated over $\mathbb{Z}[1 / 2]$.

Proof. Consider the type B graph drawn above. We apply the identity of Figure 7 to the dashed-solid chain 4-5-2-3-6-*, where $*$ is the relevant vertex of $U_{B}$. The first graph on the right side of the identity is disconnected and therefore retrogenerated. All the remaining graphs have solid-dashed doubled edges and so are also retrogenerated by Lemma 7.12. This completes the type B case. The type C case is handled similarly: in the above labeling one applies the identity of Figure 7 to the chain 4-5-2-6-3-* and then proceeds exactly as in the type B case.

The following completes the proof of Proposition 7.10.

Lemma 7.14. The type A relations span a subspace of $I_{L}^{(3)} / I_{L}^{\text {retro,(3) }}$ of dimension at most one over $\mathbb{Z}[1 / 2]$.

Proof. Put $U_{G}=\{1,2,3,4,5,6\}$ and let $\Gamma$ be a 3-colored graph on $L$ whose restriction to $U_{G}$ is the type A graph

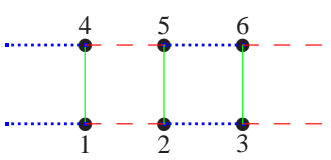


Applying the Plücker relation to the edges $\overline{45}$ and $\overline{12}$ gives an expression for $\Gamma$ in terms of two other graphs $\Gamma_{1}$ and $\Gamma_{2}$, one of which (say $\Gamma_{1}$ ) is degenerate. Now apply the Plücker relation to the edges $\overline{56}$ and $\overline{23}$ of $\Gamma_{2}$. Again, this expresses $\Gamma_{2}$ in terms of two other graphs $\Gamma_{3}$ and $\Gamma_{4}$, one of which (say $\left.\Gamma_{4}\right)$ is degenerate. Thus, we see that $\operatorname{Rel}(\Sigma)$ and $\operatorname{Rel}\left(\Sigma_{4}\right)$ are scalar multiples of each other. $\operatorname{But} \operatorname{Rel}\left(\Sigma_{4}\right)$ is nothing other than $(25) \operatorname{Rel}(\Sigma)$. We thus see that $\Gamma$ and (25) $\Gamma$ yield the same relation (up to a scalar). By similar reasoning, we see that (14) $\Gamma$ and (36) $\Gamma$ give the same relation as $\Gamma$.

Now consider the Plücker relation on $\Gamma$ on the edges $\overline{12}$ and $\overline{3 *}$, where $*$ is the relevant vertex of $U_{B}$. This results in two graphs $\Gamma_{1}$ and $\Gamma_{2}$. Say $\Gamma_{1}$ is the graph which has the edge $\overline{1 *}$. Then $\Gamma_{1}$ is a type $\mathrm{B}$ graph and thus retrogenerated. Now apply the Plücker relation on the edges $\overline{25}$ and $\overline{36}$ to $\Gamma_{2}$ to get two new graphs $\Gamma_{3}$ and $\Gamma_{4}$. One of these, say $\Gamma_{3}$, has a solid-dotted doubled edge and is thus retrogenerated. The other, $\Gamma_{4}$, is just (23) $\Gamma$. We thus see that $\Gamma$ and $(23) \Gamma$ give the same relation, up to a scalar. Similar reasoning shows that $(45) \Gamma,(56) \Gamma$ and (12) $\Gamma$ give the same relation as $\Gamma$.

From the previous two paragraphs, we see that if $\sigma$ is any permutation of $U_{G}$ then $\Gamma$ and $\sigma \Gamma$ give the same relation, up to a scalar. Since every type A graph is of the form $\sigma \Gamma$ for some $\sigma$, this establishes the lemma.

\section{The ideal is generated by quadratics}

The goal of $\S 8$ is to prove that $I_{L}$ is generated by quadratics if $|L| \geq 8$ :

Theorem 8.1. If $L$ is an even set of cardinality at least eight then $I_{L}=Q_{L}$ over $\mathbb{Z}[1 / 12$ !].

As described in $\S 1.6$, this concludes the proof of Theorem 1.1. Thanks to our work in $\S 7$, the main remaining work is to prove three base cases:

Theorem 8.2. The ideals $I_{8}, I_{10}$ and $I_{12}$ are generated by quadratics over $\mathbb{Z}[1 / 12$ !].

This implies Theorem 8.1 (and hence Theorem 1.1) by the following inductive argument. Let $n \geq 14$ and assume the theorem has been established for even sets of cardinality less than $n$. By Theorem 7.1 we have $I_{n}=I_{n}^{\text {retro }}$. By Proposition 7.4 we know that $I_{n}^{\text {retro }}$ is generated by the outer products of cubic relations on $n-2, n-4$ and $n-6$ points with arbitrary graphs on 2, 4 and 6 points. The inductive hypothesis, and the fact that $n \geq 14$, ensures that any cubic relation on $n-2, n-4$ or $n-6$ points lies in the ideal generated by quadratics. Since outer multiplication does not increase essential degree (Proposition 7.2), we find $I_{n}^{\text {retro }}=Q_{n}$. Thus Theorem 8.1 is established by induction.

As for Theorem 8.2, quadratic generation of $I_{8}$ is proved over $\mathbb{Z}[1 / 3]$ in [HMSV3]. (Quadratic generation of $I_{8}$ over $\mathbb{Q}$ had previously been established by computer; see e.g., [Ko] or [FS, Lemma 1.1].) We handle the 10- and 12-point cases by showing that $I_{10}$ (resp. $I_{12}$ ) is generated by relations coming from 8 (resp. 8 and 10) points: one does not need relations coming from six points to generate $I_{L}$. The quadratic generation of $I_{8}$ then implies that of $I_{10}$ and hence of $I_{12}$ as well. 
Here is an overview of the argument. We let $I^{\prime}$ be the quotient of the cubic part of the ideal by quadratic relations and relations retrogenerated from 8 (resp. 8 and 10) points. We wish to show that this space is zero. By Theorem 7.1, $I^{\prime}$ is generated as an $\mathfrak{S}_{10^{-}}$ module (resp. $\mathfrak{S}_{12}$-module) by a single relation: the outer product of the Segre cubic on six points by a benzene 4-cycle (resp. 6-cycle). (Hence we only need to prove that these two relations are generated by quadratics. This could in principle be checked by computer, but with current technology would probably require an algorithm of equal difficulty to our proof!) We can thus write $I^{\prime}$ as a quotient of a representation $V$ induced from $\mathfrak{S}_{6} \times \mathfrak{S}_{4}$ (resp. $\mathfrak{S}_{6} \times \mathfrak{S}_{6}$ ), where the first factor acts on the Segre cubic on six points and the second on the benzene cycle. We then write down a family of elements in the kernel of $V \rightarrow I^{\prime}$, which allows us to obtain an upper bound on the dimension of $I^{\prime}$. Finally, we examine the irreducible $\mathfrak{S}_{10}$-modules (resp. $\mathfrak{S}_{12}$-modules) occurring in $V$ and find that they all have larger dimension than the bound found for $I^{\prime}$. This shows that $I^{\prime}$ is zero. The fact that we use the representation theory of $\mathfrak{S}_{10}$ and $\mathfrak{S}_{12}$ is the source of the denominator 12 ! in Theorem 8.1.

Throughout $\S 8, L$ will always denote a set of cardinality 10 or 12 . We handle the two cases simultaneously as much as possible. When we make different statements for the two cases, we give the 10-point statement first and the 12-point statement second.

\subsection{The spaces $I_{\mathscr{P}}^{\prime}$ and the $A$-, $B$ - and $C$-relations}

By a bipartition of $L$ we mean an ordered pair $\mathscr{P}=(P, Q)$ with $P \amalg Q=L$, where $P$ has cardinality six, and $Q$ has cardinality four if $|L|=10$, and cardinality six if $|L|=12$. By a graph of type $A$ (resp. type $B$, resp. type $C$ ) with respect to a bipartition $\mathscr{P}$ we mean one of the form

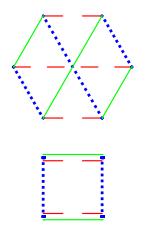

respectively

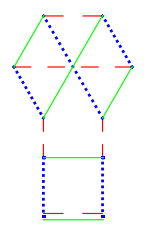

respectively

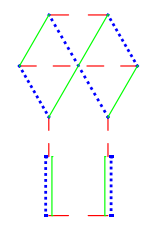

when $|L|=10$, or one of the form

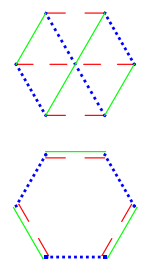

respectively

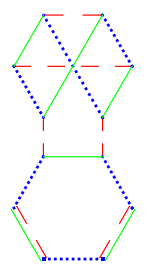

respectively

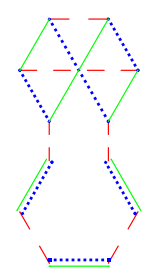

when $|L|=12$. In all cases the vertices of the top hexagon belong to $P$ while the vertices in the bottom square or hexagon belong to $Q$.

We name some edges that we will refer to often. The most obvious distinguished edges are the two dashed edges joining the $P$-vertices and $Q$-vertices. We call them the special dashed edges. There is a unique dotted (resp. solid) edge in the top hexagon meeting one 
of the special dashed edges; we call it the (top) special dotted (resp. solid) edge. Finally, for type B graphs, we call the solid edge in the bottom hexagon which meets the special dashed edges the bottom special solid edge. We call the chain $a-b-c-d$, where $\overline{a b}$ is the special dotted edge, $\overline{b c}$ is the special dashed edge to which it connects and $\overline{c d}$ is the bottom special solid edge, the special chain (only defined for type B graphs).

Let $\Gamma$ be a graph of type $\mathrm{A}, \mathrm{B}$ or $\mathrm{C}$ with respect to $\mathscr{P}$. Define a partition $\mathscr{U}=$ $\left\{U_{R}, U_{G}, U_{B}\right\}$ of $L$ as follows. In the type A case, $U_{R}, U_{G}$ and $U_{B}$ each contain two vertices in the top hexagon, and these vertices must be connected by a dashed, solid and dotted edge respectively. The bottom benzene cycle is in $U_{B}$. In the type $\mathrm{B}$ and $\mathrm{C}$ cases we take $U_{G}$ to be the two vertices belonging to the top special solid edge, we take $U_{B}$ to be the two vertices belonging to the top special dotted edge together with the bottom set (that is, the set $Q$ ), and we take $U_{R}$ to be the leftover two vertices in the top hexagon (they form a dashed edge). In each case, $(\Gamma, \mathscr{U})$ is a generalized Segre cubic datum, and so has an associated generalized Segre cubic relation. In the type A case the partition $\mathscr{U}$ was not uniquely determined, but its image in $I_{L}^{\prime}$ is independent of the choice of partition. We may thus in all cases speak unambiguously of the relation defined by $\Gamma$, denoted by $\operatorname{Rel}(\Gamma)$. Note that if $\sigma$ is a permutation of $L$ then $\operatorname{Rel}(\sigma \Gamma)=\epsilon(\sigma) \sigma(\operatorname{Rel}(\Gamma))$.

We let $I_{\mathscr{P}}^{\prime}$ be the subspace of $I_{L}^{\prime}$ generated by the relations attached to all type A graphs with respect to $\mathscr{P}$. Now, $I_{\mathscr{P}}^{\prime}$ carries a natural representation of $\mathfrak{S}_{P} \times \mathfrak{S}_{Q}$. As such it is a quotient of the representation $I_{P}^{(3)} \otimes \operatorname{gr}_{4, Q}^{(3)}\left(\right.$ resp. $I_{P}^{(3)} \otimes \operatorname{gr}_{6, Q}^{(3)}$ ). In particular, it is either zero or irreducible of dimension one (resp. five), as $I_{P}^{(3)}$ is irreducible of dimension one and $\operatorname{gr}_{4, Q}^{(3)}\left(\right.$ resp. $\operatorname{gr}_{6, Q}^{(3)}$ ) is irreducible of dimension one (resp. five; see Propositions 6.13 and 6.14). The natural map (of $\mathfrak{S}_{L}$-modules)

$$
\bigoplus_{\mathscr{P}} I_{\mathscr{P}}^{\prime} \rightarrow I_{L}^{\prime}
$$

is surjective since $I_{L}$ is retrogenerated (Theorem 7.1) and there are no relations on two or four points.

Proposition 8.3. Let $\mathscr{P}$ be a bipartition of $L$. Then the type $B$ relations with respect to $\mathscr{P}$ belong to $I_{\mathscr{P}}^{\prime}$ and span it. The same is true for the type $C$ relations.

We prove this proposition by showing that type A relations can be written in terms of type $\mathrm{B}$ relations, type $\mathrm{B}$ in terms of type $\mathrm{C}$ and finally type $\mathrm{C}$ in terms of type $\mathrm{A}$, all with respect to the same $\mathscr{P}$. We accomplish this in a series of lemmas. After this proof we will have no need for the type $\mathrm{C}$ relations.

Lemma 8.4. Any type A relation with respect to $\mathscr{P}$ belongs to the space spanned by the type $B$ relations with respect to $\mathscr{P}$.

Proof. Given a type A graph, Plücker a dashed edge from the top hexagon and a dashed edge from the bottom benzene cycle. This expresses the type A relation as a sum of two type $\mathrm{B}$ relations.

Lemma 8.5. Any type $B$ relation with respect to $\mathscr{P}$ belongs to the space spanned by the type $C$ relations with respect to $\mathscr{P}$. 
Proof. To establish this lemma we just have to rewrite the dotted-solid graph on $Q$ in terms of squares of matchings. This is exactly what we did in when proving Theorem 7.1(a) (see in particular Lemmas 7.7 and 7.8). The key point is that all square rotation relations which come up in our particular case have special paths of even length and are therefore quadratic.

Alternatively, one can argue as follows. The generalized Segre relation associated to graphs of type B is a genuine cubic relation, that is, the light-dark graph on the right side of the relation factors into a product of degree one graphs. For instance, in the 12-point case the factored relation may be written as

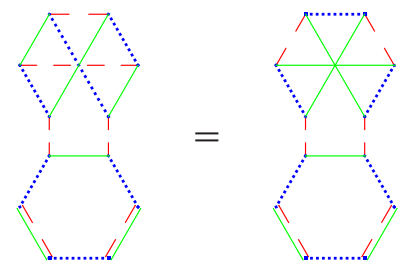

(the dark graph on the right is colored dotted while the light graph has been factored into the solid and dashed graphs). Now, the bottom half of each side is the same. One can therefore apply the same colored Plücker and quadratic relations on each side and rewrite the dotted-solid graph on $Q$ as a sum of squares of matchings. This expresses the type B relation in terms of type $\mathrm{C}$ relations.

Lemma 8.6. Any type $C$ relation with respect to $\mathscr{P}$ belongs to the space spanned by the type A relations with respect to $\mathscr{P}$.

Proof. The proof of the 10-point case proceeds exactly as the proof of Lemma 7.11. Note that after applying the identity of Figure 9 to the redrawn graph, the first graph on the second line of the right side is a type A graph with respect to $\mathscr{P}$. All other graphs either come from eight points or else are degenerate. To prove the 12-point case, we again use the identity of Figure 9, applying it to the bottom four dashed edges, that is, the two special dashed edges and the two dashed edges contained in the bottom hexagon. The first term in Figure 9 is a type A relation with respect to $\mathscr{P}$. All the other terms come from eight or ten points.

\subsection{Relations among different $I_{\mathscr{P}}^{\prime}$}

In this section we demonstrate some linear dependencies between the various spaces $I_{\mathscr{P}}^{\prime}$ :

Proposition 8.7. Let $\Gamma$ be a type A graph with respect to a bipartition $\mathscr{P}=(P, Q)$, let $a$ and $b$ be two distinct elements of $P$ and let $c$ and $d$ be two distinct elements of $Q$ which are joined by a doubled edge. Then $\operatorname{Rel}(\Gamma)$ is contained in $\sum I_{\sigma}^{\prime} \mathscr{P}$, where the sum is taken over those permutations $\sigma$ of $\{a, b, c, d\}$ for which $\sigma \mathscr{P} \neq \mathscr{P}$.

We deduce this proposition from the following one: 
Proposition 8.8. Let $\Gamma$ be a type $B$ graph with respect to $\mathscr{P}$ and let $a-b-c-d$ be its special chain. Then

$$
\operatorname{Rel}(\Gamma)=(a d) \operatorname{Rel}(\Gamma)-(b c) \operatorname{Rel}(\Gamma)+(a d)(b c) \operatorname{Rel}(\Gamma)+\operatorname{Rel}(\Delta)
$$

where $\Delta$ is a type A graph with respect to $(b d) \mathscr{P}$. In particular, $\operatorname{Rel}(\Gamma)$ belongs to $\sum I_{\sigma \mathscr{P}}^{\prime}$, the sum taken over those permutations $\sigma$ of $\{a, b, c, d\}$ for which $\sigma \mathscr{P} \neq \mathscr{P}$.

Proof of Proposition 8.7 given Proposition 8.8. Let $\Gamma, a, b, c$ and $d$ be given as in the statement of Proposition 8.7. The vertices $c$ and $d$ are connected by dashed and solid edges. Now, using a quadratic relation we may recolor the $P$-part of $\Gamma$ so that $a$ and $b$ are connected by a dotted edge. Since $\mathfrak{S}_{P}$ acts on the Segre cubic relation on $P$ via the sign character, all dotted edges in $P$ are more or less the same so we may draw $\Gamma$ as:

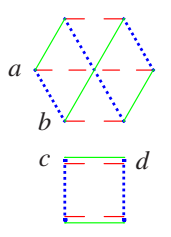

respectively
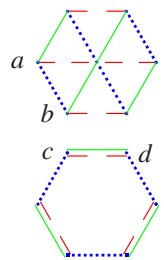

We now Plücker the dashed edge joining $c$ and $d$ and the unique dashed edge containing $b$. We obtain an expression $\Gamma=\Gamma_{1}+\Gamma_{2}$ where each $\Gamma_{I}$ is a type $\mathrm{B}$ graph with respect to $\mathscr{P}$. The special chain in $\Gamma_{1}$ is $a-b-c-d$ while in $\Gamma_{2}$ it is $a-b-d-c$. By Proposition 8.8, both $\operatorname{Rel}\left(\Gamma_{1}\right)$ and $\operatorname{Rel}\left(\Gamma_{2}\right)$ belong to $\sum I_{\sigma \mathscr{P}}^{\prime}$, where the sum is over those permutations $\sigma$ of $\{a, b, c, d\}$ for which $\sigma \mathscr{P} \neq \mathscr{P}$. Thus $\operatorname{Rel}(\Gamma)$ belongs to this space as well, which completes the proof.

We now begin proving Proposition 8.8. Consider a type B graph $\Gamma$. For convenience, we label it:

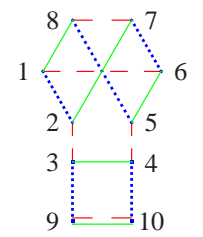

respectively

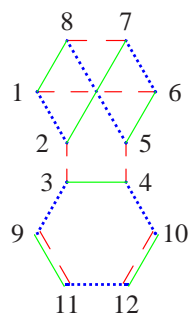

With this labeling, the special chain $a-b-c-d$ is $1-2-3-4$. We let $\mathscr{P}$ be the relevant bipartition.

By Plückering the edges $\overline{27}$ and $\overline{34}$ we obtain $\Gamma=-\Gamma_{2}-\Gamma_{3}$ where $\Gamma_{2}$ and $\Gamma_{3}$ are:
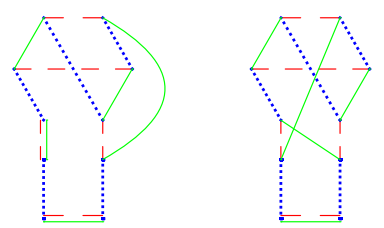

respectively
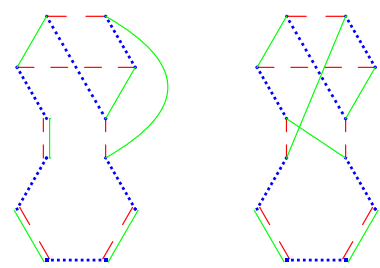
We now Plücker the edges $\overline{12}$ and $\overline{39}$ in $\Gamma_{3}$ to obtain a relation $\Gamma_{3}=-\Gamma_{4}-\Gamma_{5}$ where $\Gamma_{4}$ and $\Gamma_{5}$ are given by:
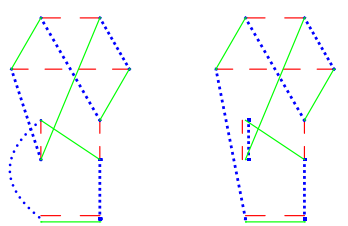

respectively
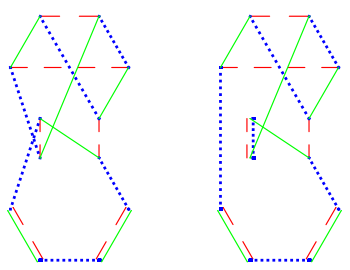

Now, we have $\Gamma_{4}=(23) \Gamma$. Thus (as generalized Segre data)

$$
\Gamma-(23) \Gamma+\Gamma_{2}=\Gamma_{5} .
$$

Lemma 8.9. We have (14) $\operatorname{Rel}\left(\Gamma_{2}\right)=\operatorname{Rel}\left(\Gamma_{2}\right)+\operatorname{Rel}(\Delta)$ where $\Delta$ is a type A graph with respect to (24) $\mathscr{P}$.

Proof. The graph $\Gamma_{2}$ has a benzene chain with three (resp. four) dotted edges, beginning at vertex 1 and ending at vertex 4 . The picture is thus:

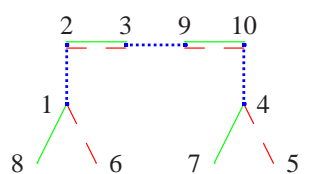

respectively

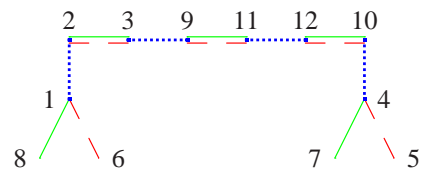

Note that $U_{R}=\{7,8\}$ and $U_{G}=\{5,6\}$ while $U_{B}$ consists of the remaining vertices.

We now Plücker the edges $\overline{81}$ and $\overline{74}$. In the first term, 8 connects to 4 and 7 to 1 . The second term is a degenerate Segre datum (since $\overline{81}$ and $\overline{74}$ are the special solid edges in $U_{B}$ ). It is thus quadratic and can be ignored. We next Plücker the edges $\overline{61}$ and $\overline{54}$, the results being similar. We have thus shown that $\operatorname{Rel}\left(\Gamma_{2}\right)$ defines the same element of $I_{L}^{\prime}$ as the relation associated to following graph (since we used two Plücker relations, no sign is introduced):

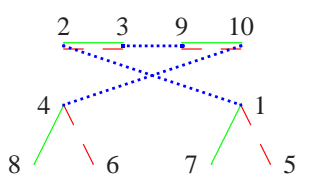

respectively

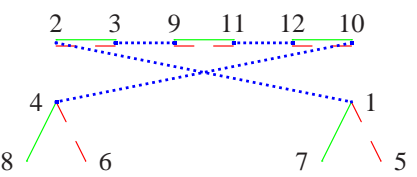

We now Plücker $\overline{12}$ and $\overline{410}$. The first term is $(14) \Gamma_{2}$. The second term is a type A graph $\Delta$ with respect to (24) $\mathscr{P}$. We thus find $\operatorname{Rel}\left(\Gamma_{2}\right)=-\operatorname{Rel}\left((14) \Gamma_{2}\right)-\operatorname{Rel}(\Delta)$, which establishes the proposition.

Lemma 8.10. We have (14) $\operatorname{Rel}\left(\Gamma_{5}\right)=\operatorname{Rel}\left(\Gamma_{5}\right)$.

Proof. We redraw $\Gamma_{5}$ in a more convenient way:

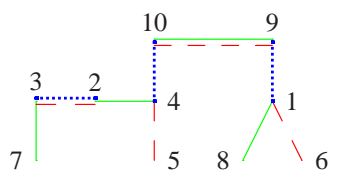

respectively

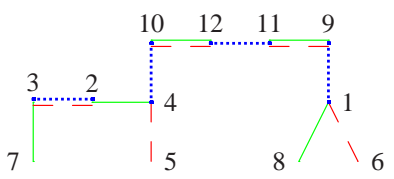

As before, $U_{R}=\{7,8\}$ and $U_{G}=\{5,6\}$ while $U_{B}$ consists of the remaining vertices. 
We now Plücker the edges $\overline{61}$ and $\overline{54}$. In the first term, 5 connects to 1 and 6 to 4 . The second term is a degenerate Segre datum and can be ignored. We next Plücker $\overline{24}$ with $\overline{81}$. In the first term, 8 connects to 4 and 2 to 1 . The second term is degenerate and can be ignored. We have thus shown that $\operatorname{Rel}\left(\Gamma_{5}\right)$ defines the same element of $I_{L}^{\prime}$ as the relation associated to the following graph:

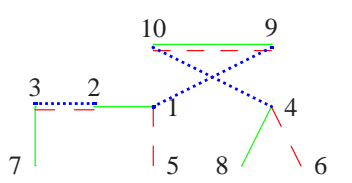

respectively

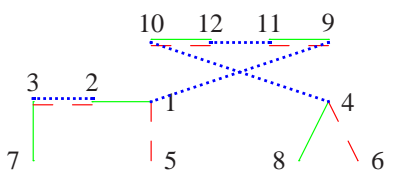

We now Plücker the edges $\overline{19}$ and $\overline{410}$. The first term is just (14) $\Gamma_{5}$. The second term is retrogenerated from eight (resp. ten) points and therefore does not contribute in $I_{L}^{\prime}$. We thus have $\operatorname{Rel}\left(\Gamma_{5}\right)=-\operatorname{Rel}\left((14) \Gamma_{5}\right)$, which establishes the proposition.

We can now complete the proof of Proposition 8.8.

Proof of Proposition 8.8. By Lemma 8.10 and (8.1), we see that $\operatorname{Rel}(\Gamma)-\operatorname{Rel}((23) \Gamma)+$ $\operatorname{Rel}\left(\Gamma_{2}\right)$ is invariant under $(14)$, that is,

$$
\operatorname{Rel}(\Gamma)-\operatorname{Rel}((23) \Gamma)+\operatorname{Rel}\left(\Gamma_{2}\right)=(14) \operatorname{Rel}(\Gamma)-(14) \operatorname{Rel}((23) \Gamma)+(14) \operatorname{Rel}\left(\Gamma_{2}\right) .
$$

We now apply Lemma 8.9 and write $(14) \operatorname{Rel}\left(\Gamma_{2}\right)=\operatorname{Rel}\left(\Gamma_{2}\right)+\operatorname{Rel}(\Delta)$ where $\Delta$ is a type A graph with respect to (24) $\mathscr{P}$. The $\Gamma_{2}$ terms on each side of the equation cancel, and we are left with

$$
\operatorname{Rel}(\Gamma)=(14) \operatorname{Rel}(\Gamma)-(23) \operatorname{Rel}(\Gamma)+(14)(23) \operatorname{Rel}(\Gamma)+\operatorname{Rel}(\Delta) .
$$

This completes the proof.

\subsection{Proof of Theorem 8.2}

We now complete the proof of Theorem 8.2. As we have explained, to do this it suffices to show that $I_{L}^{\prime}=0$. To do this we first use Proposition 8.7 to obtain an upper bound for the dimension of $I_{L}^{\prime}$, and then we use representation theory to show that this upper bound forces $I_{L}^{\prime}$ to be zero.

Fix an order on $L$. For $n=10$ (resp. $n=12$ ), we say that a bipartition $\mathscr{P}=(P, Q)$ of $L$ is good if there is at most one (resp. if there are at most two) element(s) of $Q$ which are larger than the second smallest element of $P$.

Proposition 8.11. The natural map $\bigoplus I_{\mathscr{P}}^{\prime} \rightarrow I^{\prime}$ is surjective, where the sum is taken over all good bipartitions $\mathscr{P}$.

Proof. We handle the two cases $n=10$ and 12 separately, for the sake of clarity. We begin with the 10-point case. Let $\mathscr{P}$ be a bipartition which is not good. Let $a<b$ be the smallest two elements of $P$ and let $c<d$ be the largest two elements of $Q$. Since $\mathscr{P}$ is not good, we have $a<b<c<d$. Now let $\Gamma$ be a type A graph with respect 
to $\mathscr{P}$. Since $I_{P}^{(3)} \otimes \mathrm{gr}_{4, Q}^{(3)}$ is one-dimensional, if $\Gamma^{\prime}$ is any other type A graph with respect to $\mathscr{P}$ then we have $\operatorname{Rel}(\Gamma)=\operatorname{Rel}\left(\Gamma^{\prime}\right)$. Thus we may assume that $c$ and $d$ are connected by a doubled edge in $\Gamma$. We may then apply Proposition 8.7 to conclude that $\Gamma$ belongs to $\sum I_{\sigma \mathscr{P}}^{\prime}$, the sum taken over those permutations $\sigma$ of $\{a, b, c, d\}$ for which $\sigma \mathscr{P} \neq \mathscr{P}$. Each of these $\sigma \mathscr{P}$ is closer to being good than $\mathscr{P}$ (measured by how many elements of $Q$ are larger than the second smallest element of $P$, for instance). By induction, we deduce the proposition.

We now handle the case where $L$ has cardinality 12 . Let $\mathscr{P}$ be a bipartition which is not good. Let $a<b$ be the smallest two elements of $P$ and let $c<d<e$ be the largest three elements of $Q$. Since $\mathscr{P}$ is not good, we have $a<b<c<d<e$. Now let $\Gamma$ be a type A graph with respect to $\mathscr{P}$. By Proposition 6.14(d) we can rewrite $\operatorname{Rel}(\Gamma)$ in terms of $\operatorname{Rel}\left(\Gamma_{i}\right)$, where each $\Gamma_{i}$ is a type A graph with respect to $\mathscr{P}$ in which either $\overline{c d}$ or $\overline{c e}$ appears as a doubled edge. We may as well then just assume that $\Gamma$ has $\overline{c d}$ as a doubled edge (the $\overline{c e}$ argument is the same). We now apply Proposition 8.7 and find that $\operatorname{Rel}(\Gamma)$ belongs to $\sum I_{\sigma \mathscr{P}}^{\prime}$ as $\sigma$ varies over those permutations of $\{a, b, c, d\}$ for which $\sigma \mathscr{P} \neq \mathscr{P}$. As before, each $\sigma \mathscr{P}$ is closer to being good than $\mathscr{P}$ and so we deduce the proposition by induction.

Proposition 8.12. For $n=10$ (resp. $n=12)$ there are 25 (resp. 112) good bipartitions of $L$.

Proof. We again consider the two cases separately, and begin with the 10-point case. Identify $L$ with $\{1, \ldots, 10\}$. Consider bipartitions of $L$ for which the second smallest element of $P$ is $x$. Of course, we must have $x \leq 6$. On the other hand, if $x \leq 4$ then at least two elements of $Q$ will be larger than $x$ and so the bipartition will not be good. Thus we must have $x=5$ or $x=6$.

- $x=6$. In this case, $P$ must contain each of 7, 8, 9 and 10. In addition, $P$ contains one number less than 6 . There are five such choices, each of which gives a good bipartition.

- $x=5$. In this case, $P$ must contain each of $6,7,8,9$ and 10 except for one (it cannot contain them all for then $x$ would not be second smallest). There are five ways to choose what to omit. In addition, $P$ must contain one number less than 5 . There are four such choices. Thus, all told, there are 20 good bipartitions.

Thus in total we have $5+20=25$ good bipartitions.

We now handle the case where $L$ has cardinality 12 . Again, we identity $L$ with $\{1, \ldots, 12\}$ and consider bipartitions for which the second smallest element of $P$ is $x$. We must have $x \leq 8$. Now, if $x \leq 5$ then $Q$ necessarily has three elements larger than $x$ and so the bipartition is not good. Thus we only need to consider the cases $x=6,7,8$.

- $x=8$. In this case, $P$ must contain each of $9,10,11$ and 12 . In addition, $P$ contains one number less than 8 . There are seven choices for such a number, and all give good bipartitions.

- $x=7$. In this case, $P$ must contain each of $8,9,10,11$ and 12 except for one (it cannot contain them all for then $x$ would not be second smallest). There are five ways to choose what to omit. Furthermore, $P$ must contain one element smaller than 7 . There are six such choices. Thus there are 30 good bipartitions in this case. 
- $x=6$. In this case, $P$ must contain each of 7, 8, 9, 10, 11 and 12 except for two. There are 15 ways to omit two of these numbers. Furthermore, $P$ must contain one element smaller than 6 . There are five such choices. Thus there are 75 good bipartitions in this case.

Thus in total we have $7+30+75=112$ good bipartitions.

Corollary 8.13. For $n=10$ (resp. $n=12)$ we have $\operatorname{dim} I_{L}^{\prime} \leq 25$ (resp. $\left.\leq 560\right)$.

Proof. We know that $I_{L}^{\prime}$ admits a surjection from $\bigoplus I_{\mathscr{P}}^{\prime}$, the sum taken over the good bipartitions $\mathscr{P}$. When $L$ has cardinality 10 , there are 25 of these bipartitions and each $I_{\mathscr{P}}^{\prime}$ has dimension at most one. When $L$ has cardinality 12 , there are 112 of these bipartitions and each $I_{\mathscr{P}}^{\prime}$ has dimension at most five. This gives the result.

Proposition 8.14. For $n=10$ (resp. $n=12$ ) the space $\bigoplus I_{\mathscr{P}}^{\prime}$ (summed over all bipartitions $\mathscr{P}$ ) is either zero or a direct sum of two irreducible $\mathfrak{S}_{L}$-representations of dimensions 84 and 126 (resp. three irreducible representations of dimensions 616, 1925 and 2079).

Proof. The space $\bigoplus I_{\mathscr{P}}^{\prime}$ is isomorphic as an $\mathfrak{S}_{L}$-module to $\operatorname{Ind}_{\mathfrak{S}_{P} \times \mathfrak{S}_{Q}}^{\mathfrak{S}_{L}} I_{\mathscr{P}}^{\prime}$ for any fixed bipartition $\mathscr{P}=(P, Q)$. We now handle the two cases separately.

First suppose $n=10$. Then $I_{\mathscr{P}}^{\prime}$ is either zero or isomorphic as an $\left(\mathfrak{S}_{P} \times \mathfrak{S}_{Q}\right)$ module to $I_{P}^{(3)} \otimes \operatorname{gr}_{4, Q}^{(3)}$. As an $\mathfrak{S}_{P}$-module $I_{P}^{(3)}$ corresponds to the partition $1+1+1+$ $1+1+1$, (the alternating representation) while as an $\mathfrak{S}_{Q}$-module $\mathrm{gr}_{4, Q}^{(3)}$ corresponds to the partition 4 (the trivial representation). We now use the Littlewood-Richardson rule to compute Ind $\mathfrak{S}_{\mathfrak{S}_{P} \times \mathfrak{S}_{Q}}^{\mathfrak{S}_{L}} I_{\mathscr{P}}^{\prime}$. Excluding the case where $I_{\mathscr{P}}^{\prime}=0$, we find that the induction decomposes into a direct sum of two irreducibles, corresponding to the partitions

$$
5+1+1+1+1+1, \quad 4+1+1+1+1+1+1 .
$$

By the hook length formula these irreducibles have dimensions 126 and 84, respectively.

Now say $n=12$. Again, $I_{\not P}^{\prime}$ is either zero or isomorphic as an $\left(\mathfrak{S}_{P} \times \mathfrak{S}_{Q}\right)$-module to $I_{P}^{(3)} \otimes \operatorname{gr}_{6, Q}^{(3)}$. The representation $I_{P}^{(3)}$ corresponds to the partition $1+1+1+1+1+1$ while $\operatorname{gr}_{6, Q}^{(3)}$ corresponds to $3+3$. The Littlewood-Richardson rule shows that the induction decomposes into three irreducibles, corresponding to the partitions

$4+3+1+1+1+1+1, \quad 4+4+1+1+1+1, \quad 3+3+1+1+1+1+1+1$.

These irreducibles have dimensions 2079, 1925 and 616, respectively.

We now complete the proof of Theorem 8.2.

Proof of Theorem 8.2. We must show $I_{L}^{\prime}=0$. If $n=10$, we know that on one hand, $I_{L}^{\prime}$ is at most 25-dimensional, while on the other, it is a direct sum of at most two irreducibles of dimensions 84 and 126. It follows that $I_{L}^{\prime}$ must be zero. Similarly, when $n=12$, we know that, on one hand, $I_{L}^{\prime}$ is at most 560-dimensional, while on the other, it is a direct sum of at most three irreducibles of dimensions 616, 1925 and 2079. Again, we conclude $I_{L}^{\prime}=0$. 


\section{The quadratics are generated by the simplest binomials}

In $\S 9$, we prove the following, concluding the proof of Theorem 1.2:

Theorem 9.1. If $L$ has even cardinality $n$, then the simplest binomial relations span $I_{L}^{(2)}$ over $\mathbb{Z}[1 / n !]$.

The proof uses the simple binomial relations. We show that the spans of the simple binomial relations and simplest binomial relations coincide. We then use the $\mathfrak{S}_{L}$-module structure of $I_{L}^{(2)}$ to show that simple binomial relations generate it. It suffices to prove the result over any field of characteristic 0 or greater than $n$, so we do this. (We always assume that the base field is not of characteristic 2.)

\subsection{Simple binomial relations}

Let $L$ be an even set. A binomial quadratic datum is a pair $D=(\Gamma, U)$ where $\Gamma$ is an undirected regular 2-colored graph on $L$ and $U$ is a subset of $L$ such that all edges of $\Gamma$ are contained in $U$ or $L \backslash U$. Define $\Gamma^{\prime}$ to be the graph obtained by inverting the colors of the edges of $\Gamma$ contained in $U$, and set

$$
\operatorname{Rel}(D)=Y_{\Gamma}-Y_{\Gamma^{\prime}},
$$

which is clearly a relation.

Let $D$ be a binomial quadratic datum. Suppose that a pair of edges $e$ and $e^{\prime}$ of $\Gamma$ have the same color and both lie in $U$ or $L \backslash U$. Let $\Gamma^{\prime}$ and $\Gamma^{\prime \prime}$ be the other graphs occurring in the colored Plücker relation on $e$ and $e^{\prime}$. Then $D^{\prime}=\left(\Gamma^{\prime}, U\right)$ and $D^{\prime \prime}=\left(\Gamma^{\prime \prime}, U\right)$ are binomial quadratic data. We define the space of binomial quadratic data to be the $\mathbb{Z}$-span of the binomial quadratic data modulo the (Plücker) relations $D+D^{\prime}+D^{\prime \prime}=0$. The association $D \mapsto \operatorname{Rel}(D)$ descends to a linear map

$$
\text { Rel : }\{\text { the space of binomial quadratic data }\} \rightarrow I_{L}^{(2)} \text {. }
$$

We call a binomial quadratic datum simple if $U$ has cardinality four. We call the resulting relations simple binomial relations. We say that a binomial quadratic datum is simplest if it is simple and in addition $\Gamma$ is made up of 2-cycles and two 4-cycles. The associated relations are the simplest binomial relations defined in the introduction; (1.4) is an example. Note that if $D=(U, \Gamma)$ is a binomial quadratic datum and $\Gamma$ is a union of 2-cycles and zero or one 4-cycle then $\operatorname{Rel}(D)=0$. Although there are more simple binomial relations than simplest binomial relations, they span the same space:

Proposition 9.2. Every simple binomial relation is a linear combination of simplest binomial relations over $\mathbb{Z}[1 / 2]$.

Thus to prove Theorem 9.1 it suffices to show that the simple binomial relations span $I_{L}^{(2)}$.

Proof. Let $D=(\Gamma, U)$ be a simple binomial quadratic datum. By Proposition 6.1 we can use colored Plücker relations in $L \backslash U$ to write $\left.\Gamma\right|_{L \backslash U}$ as a sum of graphs, each of which is 
a union of 2-cycles and at most one 4-cycle. This expresses $\operatorname{Rel}(D)$ in terms of simplest binomial relations.

Let $L$ be an even set and let $U$ be a subset of cardinality four. Let $L^{\prime}=L \backslash U$. Define a map

$\iota: \bigwedge^{2} V_{L^{\prime}} \otimes \bigwedge^{2} V_{U} \rightarrow I_{L}^{(2)} \quad$ by $\quad\left(Y_{\Gamma} \wedge Y_{\Gamma^{\prime}}\right) \otimes\left(Y_{\Delta} \wedge Y_{\Delta^{\prime}}\right) \mapsto Y_{\Gamma \Delta} Y_{\Gamma^{\prime} \Delta^{\prime}}-Y_{\Gamma^{\prime} \Delta} Y_{\Gamma \Delta^{\prime}}$.

This is the simple binomial relation associated to the simple binomial data $(\Phi, U)$ where $\Phi$ is the 2-colored graph $\Gamma \Gamma^{\prime} \Delta \Delta^{\prime}$ in which $\Gamma \Delta$ has one color and $\Gamma^{\prime} \Delta^{\prime}$ the other. Note that $\iota$ is $\left(\mathfrak{S}_{L^{\prime}} \times \mathfrak{S}_{U}\right)$-equivariant.

Lemma 9.3. The map $\iota$ is injective.

Proof. Let $Y_{\Delta}, Y_{\Delta^{\prime}}$ be a basis of $V_{U}$. It suffices to show that the map $\wedge^{2} V_{L^{\prime}} \rightarrow$ $\operatorname{Sym}^{2}\left(R_{L}^{(1)}\right)$ given by

$$
Y_{\Gamma} \wedge Y_{\Gamma^{\prime}} \mapsto Y_{\Gamma \Delta} Y_{\Gamma^{\prime} \Delta^{\prime}}-Y_{\Gamma^{\prime} \Delta} Y_{\Gamma \Delta^{\prime}}
$$

is injective. Let $f$ (resp. $f^{\prime}$ ) be the map $V_{L^{\prime}} \rightarrow R_{L}^{(1)}$ given by $Y_{\Gamma} \mapsto Y_{\Gamma \Delta}$ (resp. $Y_{\Gamma} \mapsto$ $Y_{\Gamma \Delta^{\prime}}$ ). Both $f$ and $f^{\prime}$ are injective. Furthermore, the images of $f$ and $f^{\prime}$ are linearly disjoint. To see this, use the fact that planar graphs form a basis for $R_{L}^{(1)}$ (Theorem 2.2): Put $L$ on a circle so that the vertices in $U$ are consecutive. If $\Gamma$ and $\Gamma^{\prime}$ are planar graphs on $L^{\prime}$ and $\Delta$ and $\Delta^{\prime}$ are distinct planar graphs on $U$ then $\Gamma \Delta$ and $\Gamma^{\prime} \Delta^{\prime}$ are distinct planar graphs on $L$. The lemma now follows from the following lemma in linear algebra.

Lemma 9.4. Let $f, g: V \rightarrow W$ be linear maps of vector spaces which are injective and have linearly disjoint images. Then the map

$$
\bigwedge^{2} V \rightarrow \operatorname{Sym}^{2}(W), \quad v \wedge w \mapsto f(v) g(w)-f(w) g(v),
$$

is also injective.

Proof. Let $v_{i}$ be a basis for $V$ and assume that $v=\sum \alpha_{i j} v_{i} \wedge v_{j}$ belongs to the kernel, so

$$
0=\sum \alpha_{i j}\left(f\left(v_{i}\right) g\left(v_{j}\right)-f\left(v_{j}\right) g\left(v_{i}\right)\right)=\sum\left(\alpha_{i j}-\alpha_{j i}\right) f\left(v_{i}\right) g\left(v_{j}\right) .
$$

The vectors $f\left(v_{i}\right) g\left(v_{j}\right)$ are linearly independent in $\operatorname{Sym}^{2}(W)$, so $\alpha_{i j}=\alpha_{j i}$ and hence $v=0$.

\subsection{Completion of the proof of Theorem 9.1}

Let $X$ denote the set of partitions of $n$ into exactly four even parts and let $X^{-}$be the set of partitions of $n-4$ into exactly four odd parts. Thus $X$ has the partitions occurring in $I_{L}$ and $X^{-}$has those occurring in $\bigwedge^{2} V_{L^{\prime}}$ (Proposition 6.5). For a partition $\lambda \in X$ we define a corresponding partition $\lambda^{-} \in X^{-}$by removing one box from each row in the Young diagram: $(a, b, c, d) \mapsto(a-1, b-1, c-1, d-1)$. This bijection of sets $X \rightarrow X^{-}$has a representation-theoretic characterization: 
Lemma 9.5. Let $\lambda^{-} \in X^{-}$and $\mu \in X$ be given. (Recall from $\$ 6.4$ that $M_{\lambda}$ is the irreducible representation of $\mathfrak{S}_{L}$ corresponding to the partition $\lambda$ ). Then

$$
\operatorname{dim} \operatorname{Hom}_{\mathfrak{S}_{L^{\prime}} \times \mathfrak{S}_{U}}\left(M_{\lambda^{-}} \otimes \epsilon, M_{\mu}\right)= \begin{cases}1 & \text { if } \lambda=\mu \\ 0 & \text { otherwise }\end{cases}
$$

Here $\epsilon$ denotes the sign representation of $\mathfrak{S}_{U}$.

Proof. By Frobenius reciprocity, the dimension of the Hom space is equal to the multiplicity of $M_{\mu}$ occurring in the induction $\operatorname{Ind}_{\mathfrak{S}_{L^{\prime}} \times \mathfrak{S}_{U}}^{\mathfrak{S}_{L}}\left(M_{\lambda^{-}} \otimes \epsilon\right)$. This can be computed using the Littlewood-Richardson rule, which is simple in this case because $\epsilon$ is just the alternating representation (the Young diagram is a single column of four boxes). The key point is that the only way to add on four boxes to $\lambda$ and end up with something in $X$ is to put one box at the end of each row.

Corollary 9.6. The map ı carries $\left(\bigwedge^{2} V_{L^{\prime}}\right)\left[\lambda^{-}\right] \otimes \bigwedge^{2} V_{U}$ into $I_{L}^{(2)}[\lambda]$ for any $\lambda \in X$, where as usual $V[\lambda]$ is the $\lambda$-isotypic part of a representation $V$ of the symmetric group.

We can now prove Theorem 9.1.

Proof of Theorem 9.1. According to the above corollary, $\iota$ induces a map

$$
\left(\bigwedge^{2} V_{L^{\prime}}\right)\left[\lambda^{-}\right] \otimes \bigwedge^{2} V_{U} \rightarrow I_{L}^{(2)}[\lambda]
$$

for any $\lambda \in X$. This map is injective (Lemma 9.3), and $\left(\bigwedge^{2} V_{L^{\prime}}\right)\left[\lambda^{-}\right]$is non-zero (Proposition 6.5). Thus the image of $\iota$ has non-zero projection to each $I_{L}^{(2)}[\lambda]$. Since $I_{L}^{(2)}$ is multiplicity-free (Proposition 6.5), the image of $\iota$ generates $I_{L}^{(2)}$ as an $\mathfrak{S}_{L}$-module. Hence the simple binomial relations span $I_{L}^{(2)}$. Since every simple binomial relation is a linear combination of simplest binomial relations (Proposition 9.2), Theorem 9.1 follows.

Acknowledgments. B. Howard was supported by NSF fellowship DMS-0703674. J. Millson was supported by the NSF grant DMS-0405606, the NSF FRG grant DMS-0554254 and the Simons Foundation. This research was partially conducted during the period A. Snowden was employed by the Clay Mathematics Institute as a Liftoff Fellow. A. Snowden was also partially supported by NSF fellowship DMS-0902661. R. Vakil was partially supported by NSF grant DMS-0801196.

We thank Shrawan Kumar, Chris Manon and Lawrence O'Neil for useful discussions.

We thank Claudiu Raicu for catching an error in an earlier version of Proposition 4.7.

\section{References}

[FS] Freitag, E., Salvati Manni, R.: The modular variety of hyperelliptic curves of genus three. Trans. Amer. Math. Soc. 363, 281-312 (2011) Zbl pre05863875 MR 2719682

[HMSV1] Howard, B., Millson, J., Snowden, A., Vakil, R.: The equations for the moduli space of $n$ points on the line. Duke Math. J. 146, 175-226 (2009) Zbl 1161.14033 MR 2477759 
[HMSV2] Howard, B., Millson, J., Snowden, A., Vakil, R.: The relations among invariants of points on the projective line. C. R. Math. Acad. Sci. Paris 347, 1177-1182 (2009) Zbl 1174.14039 MR 2566999

[HMSV3] Howard, B., Millson, J., Snowden, A., Vakil, R.: The ring of projective invariants of eight points on the line via representation theory. arXiv:0809.1233 (now subsumed into [HMSV6])

[HMSV4] Howard, B., Millson, J., Snowden, A., Vakil, R.: The ideal of relations for the ring of invariants of $n$ points on the line: integrality results. Comm. Algebra, to appear

[HMSV5] Howard, B., Millson, J., Snowden, A., Vakil, R.: http://math.stanford.edu/ ${ }^{\sim}$ vakil/HMSV/jems.html

[HMSV6] Howard, B., Millson, J., Snowden, A., Vakil, R.: The ring of projective invariants of eight points on the line via representation theory. arXiv:1103.5255, submitted

[Ke] Kempe, A.: On regular difference terms. Proc. London Math. Soc. 25, 343-350 (1894) JFM 25.0235.01

[Ko] Koike, K.: The projective embedding of the configuration space $X(2,8)$. Technical Reports of Mathematical Sciences, Chiba Univ. 16 (2000).

[Ku] Kumar, S.: A remark on universal connections. Math. Ann. 260, 453-462 (1982) Zbl 0476.55019 MR 0670193

[M] Manon, C.: Presentations of semigroup algebras of weighted trees. Algebr. Combin. 31, 467-489 (2010) Zbl pre05721554 MR 2639721

[SS] Speyer, D., Sturmfels, B.: The tropical Grassmannian. Adv. Geom. 4, 389-411 (2004) Zbl 1065.14071 MR 2071813

[S] Sturmfels, B.: Gröbner Bases and Convex Polytopes. Univ. Lecture Ser. 8, Amer. Math. Soc., Providence, RI, 1996. Zbl 0856.13020 MR 1363949

[W] Weyl, H.: The Classical Groups: Their Invariants and Representations. Princeton Univ. Press, Princeton, NJ (1997). Z Zbl 1024.20501 